\title{
Scattering amplitudes and toric geometry
}

\author{
Antonio Amariti $^{a}$ and Davide Forcella ${ }^{b}$ \\ ${ }^{a}$ Laboratoire de Physique Théorique de l'École Normale Supérieure and \\ Istitute de Physique Théorique Philippe Meyer, \\ 24 Rue Lhomond, Paris 75005, France \\ ${ }^{b}$ Physique Théorique et Mathématique and International Solvay Institutes, \\ Université Libre de Bruxelles, \\ C.P. 231, 1050 Bruxelles, Belgium \\ E-mail: amariti@lpt.ens.fr, davide.forcella@ulb.ac.be
}

ABSTRACT: In this paper we provide a first attempt towards a toric geometric interpretation of scattering amplitudes. In recent investigations it has indeed been proposed that the all-loop integrand of planar $\mathcal{N}=4 \mathrm{SYM}$ can be represented in terms of well defined finite objects called on-shell diagrams drawn on disks. Furthermore it has been shown that the physical information of on-shell diagrams is encoded in the geometry of auxiliary algebraic varieties called the totally non negative Grassmannians. In this new formulation the infinite dimensional symmetry of the theory is manifest and many results, that are quite tricky to obtain in terms of the standard Lagrangian formulation of the theory, are instead manifest. In this paper, elaborating on previous results, we provide another picture of the scattering amplitudes in terms of toric geometry. In particular we describe in detail the toric varieties associated to an on-shell diagram, how the singularities of the amplitudes are encoded in some subspaces of the toric variety, and how this picture maps onto the Grassmannian description. Eventually we discuss the action of cluster transformations on the toric varieties. The hope is to provide an alternative description of the scattering amplitudes that could contribute in the developing of this fascinating field of research.

Keywords: Supersymmetric gauge theory, Scattering Amplitudes, Differential and Algebraic Geometry

ArXiv EPrint: 1305.5252 


\section{Contents}

1 Introduction 2

2 An overview: amplitudes and toric geometry 3

2.1 A simple example: three particles amplitude 9

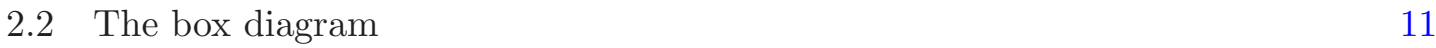

$\begin{array}{lll}3 & \text { Bipartite diagrams, polytopes and toric geometry } & \mathbf{1 5}\end{array}$

$\begin{array}{lll}3.1 & \text { Polytopes } & 16\end{array}$

$\begin{array}{lll}3.1 .1 & \text { A remark on polytopes and toric geometry } & 17\end{array}$

4 Coordinates on $\operatorname{Gr}(k, n)^{t n n}$ from bipartite graph $\quad 18$

$\begin{array}{ll}4.1 \text { Coordinates from the flows } & 19\end{array}$

$\begin{array}{lll}4.2 & \text { Coordinates from the PMs } & 19\end{array}$

5 Toric geometry and cell decomposition $\quad 24$

6 Examples $\quad \mathbf{2 6}$

6.1 $\operatorname{Gr}(1,3)^{t n n}$ and $\operatorname{Gr}(2,3)^{t n n} \quad 26$

$6.2 \operatorname{Gr}(2,4)^{\operatorname{tnn}} \quad 27$

$\begin{array}{ll}\text { 6.2.1 Gluing the patches } & 30\end{array}$

6.2.2 Cell decomposition 32

6.3 A subcell of $G r(3,6)^{\operatorname{tnn}} \quad 35$

$\begin{array}{ll}\text { 6.4 Toric ideals and cell decomposition } & 39\end{array}$

$\begin{array}{ll}\text { 6.4.1 Triangular basis } & 39\end{array}$

$\begin{array}{lll}\text { 6.4.2 The cube } & 40\end{array}$

7 Dual diagrams and cluster transformations $\quad 42$

7.1 Cluster transformations in $\operatorname{Gr}(2,4)^{\operatorname{tnn}} 44$

7.2 A subcell of $\operatorname{Gr}(3,6)^{\operatorname{tnn}} \quad 46$

$\begin{array}{lll}8 & \text { Conclusions } & 48\end{array}$

A The totally non negative Grassmannian $\quad \mathbf{5 0}$

$\begin{array}{lll}\text { A.1 Grassmannians } & 50\end{array}$

A.2 Bipartite diagrams and $G r(k, n)^{t n n} \quad 51$

A.2.1 Cell decomposition and bipartite diagrams 52

B Some further considerations

C List of notations $\quad 54$ 


\section{Introduction}

In the last years a deeper understanding of scattering amplitudes in QFTs with a high degree of symmetry was pursued. The most studied and understood example is the planar limit of $\mathcal{N}=4 \mathrm{SYM}$ in four dimensions, where, thanks to the large symmetry group, many efficient methods have been developed [1-22]. In particular an hidden symmetry of this theory has been recently discovered: the dual superconformal invariance [19, 20, 2325], that together with the usual superconformal symmetry, gives origin to the infinite dimensional Yangian symmetry of the theory.

The presence of such infinite dimensional symmetry hidden in the Lagrangian formulation of the theory, and the unexpected simplicity of some scattering amplitudes, foster the research for a "dual" formulation for its $S$-matrix, where the full symmetry group is manifest and the scattering processes and their singularities have a simple form. In $[21,22,26]$ a dual formulation was proposed in terms of the geometry of an auxiliary algebraic geometric variety called the totally non negative Grassmannian. In this formulation the information needed to reconstruct the scattering amplitudes are encoded in the combinations of residues of contour integrals of some well defined invariant form on the Grassmannian [21, 22, 26]. The space-time locality and unitarity of the theory are emergent, non explicitly imposed, properties, while the full symmetry of the theory is manifest and encoded in the algebraic structure of the Grassmannian and its decomposition in subspaces.

Quite interestingly the totally non negative Grassmannians are intimately related to a set of bipartite graphs (collections of edges connecting black and white nodes) drawn on disks [27]. These graphs are the so called on-shell diagrams describing the scattering process: all the particles in the diagrams are on-shell. Very similar bipartite graphs, but living on tori instead of disks, have played a central role in the understanding of the AdS/CFT correspondence [28-33]. They describe the field theory living on a stack of D3 brane probing some Calabi-Yau singularity with at least $\mathrm{U}(1)^{3}$ symmetries: the so called toric Calabi-Yau singularities. Recently there was some interest in the study of the bipartite graphs on Riemann surfaces other that the torus and the field theory associated to them [34-39].

The field theories defined by bipartite graph on disks, relevant for scattering processes, are special examples of this new class of theories. In particular it was shown that, as in the case of the torus, it is always possible to associate a toric variety to these field theories [34]. It is natural to wonder if these two parallel lines of research based on bipartite graphs on disks could produce interesting contributions one to the other.

Indeed for example in [40] it was observed that the mathematical structure of the two lines of research is exactly the same, supporting the idea that it is worthwhile to investigate this relationship in more detail. An important step was done in [41] where it was shown how to associate a toric variety to a given bipartite graph on the disk, that can be smoothly mapped to a specific subspace of the non negative Grassmannian, relevant for the scattering amplitudes. This important result opens the possibility of an explicit reformulation of the scattering process in terms of toric varieties. This is the line of thought that we will pursue in this paper. Indeed, motivated by this connection, in this paper we 
study the relation among bipartite graphs, the Grassmannian and its subspaces, and the toric geometry. Taking advantage of some well developed techniques available for the case of the bipartite diagrams on the tori we find a very simple global description of the toric geometry associated to the bipartite graphs on the disk and its map to the non negative Grassmannian. We study the local and global coordinates and the associated map between toric and Grassmannian geometry, the decomposition of the Grassmannian in subspaces and the associated decomposition of toric varieties in their toric subspaces, and the cluster transformations of the coordinates among different patches.

The rest of the paper is organized as follows: in section 2 we give a general overview of the connection between the scattering amplitudes and the bipartite graphs on the disk, Grassmannian geometry and toric geometry. To clarify some aspects of these connections we study two simple examples. In section 3 we start a deeper analysis of the bipartite diagrams on the disks. We define the main objects necessary to our investigations, the perfect matchings and the perfect orientations and we discuss the two polytopes, the matching and the matroid polytope, that play a key role in the relation with the amplitudes. In section 4 we derive one of the main results of our paper. We show that there is a simple system of coordinates describing the points in the Grassmannian obtained from the bipartite diagrams. In section 5 we review the relation between the bipartite graphs and toric geometry and explain the difference between the toric varieties associated to the scattering amplitudes and the ones studied in the AdS/CFT literature. In section 6 we revisit the examples under this new light, and study the amplitudes in terms of their relation with the toric varieties. In section 7 we analyze the transformation of our coordinates under a set of movement on the diagrams, usually referred as square moves (Seiberg duality in the physics literature), and we describe in this picture the associated cluster transformations. In section 8 we conclude and discuss some further directions of research in this field. In appendix A and B we review some mathematical material related to the Grassmannian and some further property of the toric geometric varieties associated to the on-shell diagrams.

\section{An overview: amplitudes and toric geometry}

The standard formulation of quantum field theory is based on the concepts of manifest locality and unitarity. However this description forces to introduce redundancies associated to the gauge symmetries and it hides some of the important structures of the field theory itself. This observation is supported by the simple final results of various complicated scattering amplitudes processes, the hidden dual conformal symmetry and the infinite dimensional Yangian symmetry of planar $\mathcal{N}=4 \mathrm{SYM}$, and, more generally, by the existence of various gauge/string dualities and gauge/gauge dualities hidden in the standard formulation of quantum field theory [19, 20, 23-25, 43-45]. Inspired by these motivations there was a recent effort in trying to reformulate the description of some of the physical processes of a quantum field theory, namely scattering amplitudes, in a setup where the concepts of locality, unitarity, Lagrangian, etc ..., do not play any fundamental role, but they are instead emergent structures of a description where all the symmetries of the theory are manifest and where all the quantities that are computed are on-shell and well defined. 
The idea to reconstruct the scattering amplitudes of a field theory starting from an onshell formulation where the symmetries are manifest and the basic objects are well defined and free of divergences is somehow a natural paradigm for a theoretical physicist, but that has been a bit obscured by all the well know difficulties of standard quantum field theory.

Along this general line of reasoning, a systematic way to reconstruct the all loops scattering amplitudes of planar $\mathcal{N}=4 \mathrm{SYM}$ was proposed in [26]. The main insight is to focus on a certain class of objects: the so called on-shell diagrams. These diagrams are obtained by gluing together elementary three particle processes, and are well defined functions of the external momenta of a scattering process. Pictorially they are very similar to the usual Feynman diagrams, but physically they are very different: all the momenta are on-shell and complex in general. The underlying intuitive understanding of this reformulation is a different way to think about permutations. Indeed it was observed that the four dimensional scattering amplitudes behave somehow similarly to the $1+1$ integrable two dimensional systems, where the scattering matrix can be reformulated in terms of permutations only. In the four dimensional case the relation between scattering amplitudes and permutations is slightly different from the case of two dimensional systems, but the bottom line is that a permutation, or a set of permutations, can be associated to a scattering process. The integrand of the scattering amplitudes can be computed from the permutations and hence the full amplitude can be reconstructed.

The first step is to observe that also in four dimensions permutations can be represented graphically by classes of on-shell two dimensional diagrams. These on-shell diagrams are unoriented bicolored graphs drawn on a disk with black and white vertices connected by edges (see for example (2.1)), and they are supposed to contain the physical information of the scattering of $n$ massless particles: $k$ with negative helicity and $n-k$ with positive one. ${ }^{1}$ The diagram has indeed $n$ external edges that end on the boundary and intuitively represent the $n$ massless particles in the scattering process. The edges represent the onshell particles involved in the process and the coloring of the vertices are related to the helicities. At computational level one should integrate over all the internal edges (complex internal momenta) and impose momentum conservation at every vertex.

In [26] a systematic way to deal with these on-shell diagrams was explained. It unifies the description of any scattering processes and it is manifestly invariant under the full symmetry of the theory. This strategy reformulates the scattering process in terms of permutations and auxiliary algebraic geometric varieties, and provides a nice new point of view to look at the quantum field theory dynamics.

The on-shell diagrams are associated to the permutations as follows. Let us first introduce a notion of path in the diagram: a path is a collection of consecutive edges in the graph; if a path turns maximally right at the black nodes and maximally left at the white ones it is called left-right path. ${ }^{2}$ Given an on-shell graph, the permutation is obtained

\footnotetext{
${ }^{1}$ What one is actually computing in the case of $\mathcal{N}=4$ SYM is a super-amplitude, where the highest order component is the amplitude for $k$ gluons with negative helicity and $n-k$ gluons with positve helicity. In all the paper we will keep on focusing on the highest component of the super-amplitudes, with the hope that the reader will not be confused by this abuse of notation.

${ }^{2}$ If the diagram is bipartite, i.e. every edge connects a black and a white node, the left-right paths are called zig-zag paths.
} 
by connecting the external vertices of the diagram with the left-right paths. These paths uniquely provide a map among the $n$ external particles. This map is a permutation of $n$ objects. The permutation provides also the number $k$ of external particles with negative helicities: this is the number of particles that are sent "beyond" $n$ by the permutation. See for example (2.1)

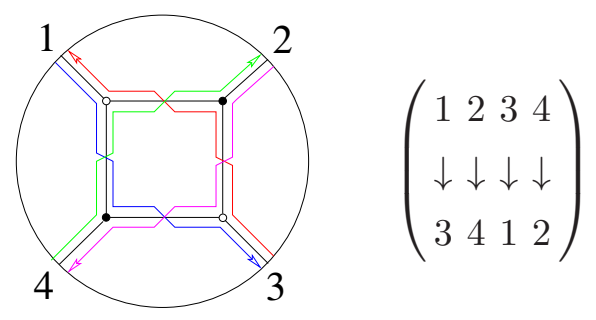

More formally the integer $k$ is defined as follows. Define a permutation of $n$ objects $a_{i}$ as a map $\Sigma:\{1, \ldots, n\} \rightarrow\{1, \ldots, 2 n\}$ that permutes $a_{i}$ with $a_{j}: \Sigma\left(a_{i}\right)=a_{j}$ if $i<j$ or $\Sigma\left(a_{i}\right)=a_{j+n}$ if $i>j$. The integer $k$ counts the number of elements that are sent beyond $n$ by the permutation. These kind of permutations are called decorated permutation of type $(k, n)$.

At this level we have just qualitatively discussed the relation between the permutations and the on-shell diagrams. At the same time we know that the on-shell diagrams should also represent scattering processes. For this reason the permutations should also encode the quantitative information of this scattering. The second step of this reformulation of scattering amplitudes is a mathematical theorem that states that a given $(k, n)$ permutation is in one to one correspondence with a specific subspace (called a "cell") in the space of $k$-dimensional planes in $\mathbb{R}^{n}$ : the so called Grassmannian $\operatorname{Gr}(k, n)$ [27].

The appearance of the Grassmannian $\operatorname{Gr}(k, n)$ in the description of a scattering amplitude process should not be a surprise. Indeed for example the space of two planes in $n$ dimensions, $\operatorname{Gr}(2, n)$, is the natural Lorentz invariant way to describe the scattering process of $n$ massless particles [21].

We will review most of the terminology about the Grassmannian in the next sections, but here we need just a few definitions. A $k$-plane in $\mathbb{R}^{n}$ is specified by $k$ vectors of $n$ components (a $n \times k$ matrix) modulo the action of the $G L(k)$ group, that moves the vectors, but it leaves the $k$-plane invariant. The $k$-minors of the $n \times k$ matrix are invariant under the SL $(k)$ group and they are simply multiplied by a common factor by the action of $G L(k)$. They provide indeed a good set of projective coordinates to describe $\operatorname{Gr}(k, n)$ : the so called Plücker coordinates. The totally non negative Grassmannian $\operatorname{Gr}(k, n)^{\operatorname{tnn}}$ (the main actor in this reformulation of the scattering amplitudes) is the subspace of $\operatorname{Gr}(k, n)$ such that all the $k$-determinants are non negative and the $G L(k)$ group is restricted to the positive numbers. The $G r(k, n)^{t n n}$ admits a simple decomposition in open subspaces, called cells, that are obtained by sending (in a precise way to be explained in the following sections) some of the Plücker coordinates to zero and forcing the others $k$-determinants to be positive. This process is called the cell decomposition of the $G r(k, n)^{t n n}$ and it 
reconstructs $\operatorname{Gr}(k, n)^{t n n}$ as the disjoint union of its cells, in a similar way as, the probably more familiar, decomposition of the projective space in the union of some of its subspaces. ${ }^{3}$

This decomposition of $G r(k, n)^{t n n}$ is somehow the algebraic geometric counterpart of the reconstruction of the scattering amplitude of $n-k$ massless particles with positive helicity and $k$ massless particles with negative helicity, from its singularities (the BCFW procedure is a famous example).

The main claim of this reformulation of scattering amplitudes is that the scattering amplitude process of $n$ particles, with $k$ of negative helicities, is somehow encoded in the decorated permutations of type $(k, n)$ that are themselves in $1-1$ correspondence with the cells in the totally non negative Grassmannian $\operatorname{Gr}(k, n)^{t n n}$. Namely to every permutation $\Sigma$, relevant to the scattering process, can be indeed associated a contour integral of the top invariant form of $G r(k, n)^{t n n}$ along some appropriate contour [26]:

$$
\mathcal{F}_{\Sigma}=\int \frac{1}{\operatorname{Vol}(G L(k))} \frac{d^{k \times n} A_{i j}}{(12 \ldots k) \ldots(n, 1, \ldots, k-1)} \prod_{i=1}^{k} \delta\left(A_{i j} Z_{j}\right)
$$

where the integral should be interpreted as a contour integral and one should compute its residues. The $n \times k$ matrix $A$ parameterizes the cells in $\operatorname{Gr}(k, n)^{t n n}$, in the denominator there are the $n$ consecutive $k$-minors of the $A$ matrix, while $Z_{j}$ are the twistor superspace variables containing the momenta of the external particles, and the $\delta$-functions impose the momentum conservation of the scattering process. The specific parametrization of $A$ that allows to associate a cell to the permutation is obtained (as explained in the following sections) by using the on-shell diagram representation of the permutation.

The full set of singularities of the top form in (2.2) have in general a quite complex structure and they are encoded in the cell decomposition of $\operatorname{Gr}(k, n)^{t n n}$, as shown in [26]. A careful parametrization of $G r(k, n)^{t n n}$ in terms of $n(n-k)$ local coordinates transforms the integrand form in $(2.2)$ in a product of $n(n-k) d$-logarithms: $d f_{i} / f_{i}$, explicitly showing that the only singularities are of the logarithmic type and that they occur at the boundary of the positive part of $G r(k, n)^{t n n}$. However this is just a local description and it can be shown that it is not possible to represent all the singularities in a single patch [26]. For this reason the full geometry of $G r(k, n)^{t n n}$, the gluing procedure of the various patches or a global description in terms of embedding coordinates, is required to obtain the scattering amplitudes and is the full variety that encodes the complete set of physical information.

In this paper we want to provide a complementary description of the scattering amplitudes in terms of toric geometry (a branch of algebraic geometry where the varieties can be simply described in terms of polytopes). We will elaborate on previous results about the existence of a map between Grassmannians and certain toric varieties in the mathematical literature [41], and the known relation between bipartite graphs and toric geometry in the physical literature [28-33]. Our aim is to provide an alternative description of the scattering process that could hopefully help in the progress of this fascinating field of research. We will show how to associate a toric variety to the scattering process and how

\footnotetext{
${ }^{3}$ It is important to observe from the very beginning that the cell decomposition we will speak about in this paper is not the usual Schubert cells decomposition but an alternative, much simpler one, called positroid decomposition [27, 46] relevant for the scattering amplitudes [26].
} 
the global description of this geometry encodes the necessary informations to reconstruct the amplitude.

Toric varieties will be introduced in the following sections. For the moment it should be enough to state that the main actor in this alternative description of the scattering amplitudes are the non negative part of real toric varieties. Roughly speaking a complex toric variety of dimension $d$ is an algebraic geometric variety that has at least a $\mathrm{U}(1)^{d}$ isometry group, its algebraic equations are of the kind monomial $=$ monomial and it can be represented as a $d$ dimensional polytope. The real non negative part of these varieties is just the restriction of the complex coordinates to their non negative real values. Toric geometry is usually a very interesting tool that provides a simple description of quite complex systems. This simple description revealed to be very useful for example in the domain of the gauge/gravity correspondence and in algebraic geometric in general. We hope that it could be useful also in the domain of the scattering amplitudes.

Toric geometry can be introduced thanks to the relation between decorated permutations and bicolored graphs. We previously stated that a permutation can be represented in terms of on-shell diagrams thanks to the left-right paths. However this representation is not unique and different on-shell diagrams are in general associated to the same permutation. Namely one class of on-shell diagrams is associated to one permutation. At a first look an infinite number of on-shell graphs could be associated to every permutation, obtained for example just by keeping on adding internal faces. However we should distinguish between "reduced" and "non-reduced" graphs. The reduced graphs are the graphs that have somehow the minimum amount of faces for a given permutation. The "non-reduced" graphs could then be obtained for example by simply adding faces to the reduced ones. The class of reduced diagrams will then contain only a finite set of graphs with the same permutation.

More formally reduced diagrams are defined as follows. First of all we should define a set of moves on the diagram. The moves are

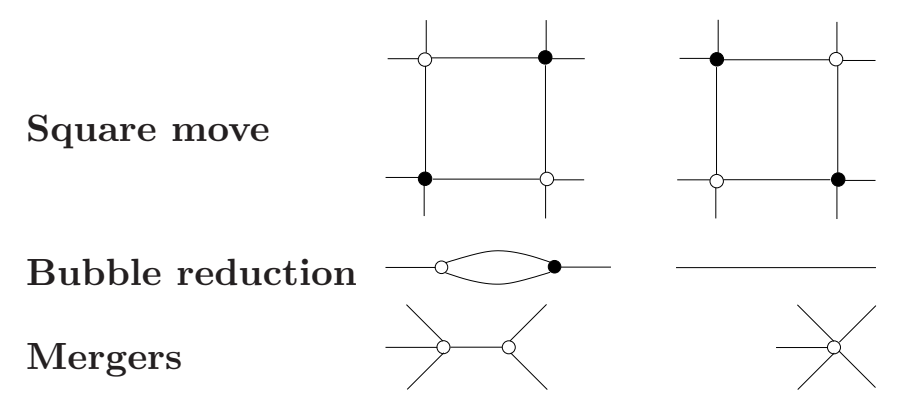

If we apply mergers and square moves to an on-shell diagram one can in principle generate a bubble on some internal line of the diagram. This bubble can be eliminated thanks to the bubble reduction. This process can be iterated until no bubble can be created anymore. The diagram obtained by this procedure is called reduced.

Among these reduced diagrams there exists a finite set associated to the same permutation and hence the same physical process. The class of reduced diagrams related to the same permutation and hence to the same cell in the Grassmannian is obtained from a reduced diagram by applying mergers and square-moves. 
The basic elements associated to a scattering amplitude are the reduced diagrams modulo the action of square move and mergers. ${ }^{4}$ By applying those transformations each bicolored graph can be put in a bipartite form, i.e. every edge connects a white vertex with a black one. The toric varieties are constructed from these bipartite graphs.

The correspondence between bipartite graphs and toric geometry may look not surprising, especially due to some important developments in the AdS/CFT correspondence. Indeed in this area there exists a class of superconformal field theories (SCFTs) that can be represented and engineered in terms of branes as bipartite diagrams drawn on a torus $\mathbb{T}^{2}$. A toric variety can be associated to each bipartite graph, modulo the action of movements similar to the square move and the mergers [41].

In the present discussion the bipartite diagrams are drawn on a disk. However recently these diagrams have been studied by mathematicians [27] and their connection with toric geometry was firstly investigated in [41].

In [41] it was shown that bipartite diagrams on the disk can be associated to projective toric varieties. First one defines the perfect matchings, PMs (from now on $\sigma_{i}$, with $i=$ $1, \ldots, c)$ : collections of edges minimally covering all the vertices in the diagram (for every vertex there is only one edge ending on it). The PMs are not generically independent, but they satisfy a set of $s$ linear relations among themselves. To every PM one associates a projective coordinate (cfrom now on $\pi_{i}$, with $\left.i=1, \ldots, c\right)$ in $\mathbb{R P}_{>0}^{c-1}$ where $c$ is the total number of PMs. The linear relations among the PMs become product relations among the associated projective coordinates (the exponential of the PM linear relations).

The closure in $\mathbb{R P}_{\geq 0}^{c}$ of these product relations, namely the addition of the limit points such that the coordinates can go to zero, defines the projective toric variety. To every PM one can also associate a vector in $\mathbb{R}^{c-s}\left(v_{\sigma_{i}}\right.$ with $\left.i=1, \ldots, c\right)$. This map is obtained by solving the set of $s$ linear relations in terms of $c-s$ basis PMs. The convex hull formed by these $c s$-vectors defining the projective toric variety is a polytope, called the matching polytope. The singularities of the form in the integrand in (2.2), described as cell decomposition in the $G r(k, n)^{t n n}$ picture, are instead described as a "partial facets decomposition" of the toric matching polytope. Namely to every singularity in the form in (2.2) is associated at least a facet in the polytope describing the toric projective variety.

More concretely let us introduce the notation $p_{I}$ for the previously introduced Plücker coordinates of $G r(k, n)^{t n n}$. I is the set of all possible $k$ edges among the possible $n$ external edges of the on-shell diagram, namely the set of all the possible $k$-minors of the $n \times k$ matrix defining a point in the Grassmannian: there are $\left(\begin{array}{l}n \\ k\end{array}\right)$ of them. These coordinates are not free, but satisfy a set of algebraic equations that define the Plücker embedding of $\operatorname{Gr}(k, n)^{\operatorname{tnn}}$ into $\mathbb{R P}\left(\begin{array}{l}n \\ k \\ k 0\end{array}\right)-1$.

Elaborating on some previous results [47] in this paper we will show that the map from the projective toric variety to the totally non negative Grassmannian, and hence to the integrand in (2.2) and its singularities, is provided by the following linear map between the

\footnotetext{
${ }^{4}$ It is however important to state that the non-reduced graphs play an important role too in particular in relation to loop amplitudes, but that they can however be reconstructed from reduced on-shell graphs.
} 
embedding coordinates of the two varieties:

$$
p_{I}=\sum_{j \in \text { internal loop }} \pi_{I}^{(j)}
$$

where $\pi_{I}^{(j)}$ is the coordinate associated to the $j$-th PM and we are summing over the PMs that only differ by internal lines. This map stands at this point at the intuitive level, and it will be formalized in detail in the following sections. As we will show later indeed PMs differing by internal lines are associated to the same set of $k$ elements that is parameterized by $I$. It is important to underline from the very beginning that the map from the non negative part of the real projective toric variety to $\operatorname{Gr}(k, n)^{t n n}$ or a closure of one of its cell, is an homeomorphism between the positive part of both varieties, but it is in general not an isomorphism on the boundaries, namely for the zero values of some of the coordinates in the toric varieties and in the Grassmannian.

This map is the global version, in a sense that will be explained later in the paper, of the one in [47].

The singularities of the on-shell diagram are obtained by the knowledge of the polynomial relations among the $\pi$ coordinates of the toric variety and the map from the $\pi$ to the coordinates $p$ of the Grassmannian. One starts from the polytope identified by the PMs and projects on the facets, by sending some edges of the on-shell diagram to zero. The edges that can be sent to zero are called removable. An edge is removable if the two leftright paths that intersect on it do not intersect on other edges in the diagram. By removing an edge one removes also some PMs, the ones that contain the edge, and reduces on some face of the polytope. Some $p$ coordinate vanishes as well. The remaining non vanishing coordinates on each facet represent the singularity. This procedure can be redundant: by sending to zero different sets of PMs we may describe the same singularity. This happens if the PMs that we send to zero differ only by internal faces. In the rest of this section we study in detail two simple examples to elucidate the procedure that we just reviewed.

\subsection{A simple example: three particles amplitude}

In this section we use the toric approach to study the on-shell diagram describing the tree level scattering of three particles, two with positive and one with negative helicity: namely the maximal helicity violating (MHV) tree level three particles amplitude. The on-shell diagram for this amplitude is represented by one of the three graphs in (2.5), where we also explicitly show the three PMs.
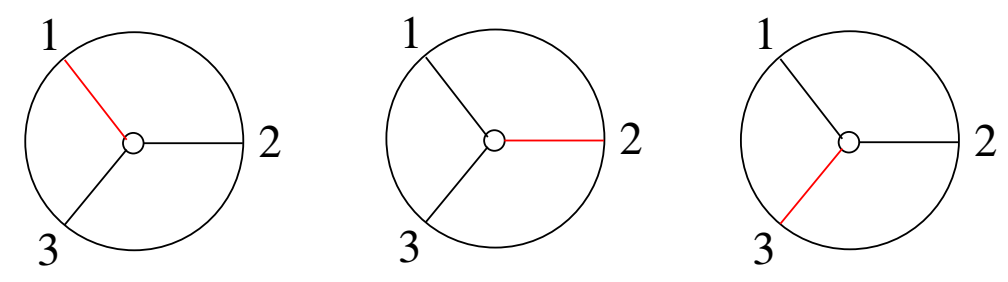

The corresponding anti MHV amplitude $(\overline{M H V})$ is obtained by inverting the color of the vertex. In both cases it should be easy to see that there are three PMs and no relations among them (every edge is a PM). 
In this very simple case, we don't have internal faces, and we do not have relations, and hence the map between the totally non negative toric variety and the totally non negative Grassmannian (2.4) should be an isomorphism and the two descriptions are totally equivalent.

Let us see explicitly how it works. The diagram in (2.5) is associated, according to the map that we have previously explained, to the totally non negative Grassmannian with $k=1$ and $n=3, \operatorname{Gr}(1,3)^{t n n}$. This is the space of lines in $\mathbb{R}^{3}$ and it is exactly the totally non negative part of the two dimensional real projective space: $\mathbb{R P}_{\geq 0}^{2}$.

This space is decomposed in cells as follows. The top dimensional subcell in the decomposition is $\mathbb{R} \mathbb{P}_{>0}^{2}$, where all the coordinates are non vanishing. The lower dimensional subspaces are three copies of $\mathbb{R P}_{>0}^{1}$, where one of the original three coordinates is vanishing, while the other two are strictly positive. The lowest dimensional cells are three points in the projective space: each one associated to a single non vanishing coordinate. Hence the full $\operatorname{Gr}(1,3)^{t n n}$, namely $\mathbb{R P}_{\geq 0}^{2}$, can be reconstructed as the union of its seven cells: $\mathbb{R P}_{\geq 0}^{2}=\mathbb{R P}_{>0}^{2} \cup 3 \mathbb{R P}_{>0}^{1} \cup 3$ points.

The same description can be obtained in terms of toric geometry. One starts from the three PMs: $\sigma_{i}$ for $i=1,2,3$, in (2.5). There are not relations among them and the toric diagram (that will be often called matching polytope in the rest of the paper) is described by the three vectors $v_{\sigma_{i}}$ :

$$
v_{\sigma_{1}}=(1,0,0) \quad, \quad v_{\sigma_{2}}=(0,1,0) \quad, \quad v_{\sigma_{3}}=(0,0,1)
$$

We can associate a totally non negative toric variety to this toric diagram in the following way. To each PM $\sigma_{i}$ we associate a coordinate $\pi_{i}$ in $\mathbb{R P}_{>0}^{2}$. The absence of relations among the $\sigma_{i}$ implies the absence of relations among the $\pi_{i}$, and we can safely take the closure of the space. We obtain in this way that the toric variety associated to the tree level three points MHV amplitude is $\mathbb{R P}_{\geq 0}^{2}$ that is exactly equal to $\operatorname{Gr}(1,3)^{t n n}$.

The $\pi$ coordinates are in 1-1 correspondence with the $p$ coordinates of the Grassmannian. From the toric diagram (2.7) we can recover the cell decomposition of $G r(1,3)^{\operatorname{tnn}}$ in terms of the decomposition into subpolytopes of the matching polytope. The various subspaces are obtained by sending to zero the various $\pi$ coordinates.

One can give a pictorial representation of this decomposition. First assign a vector on a three dimensional lattice to every PM as in formula (2.6). This identifies a multidimensional polytope. These vectors lie on a plane and we can restrict to the basis of the polytope. By eliminating a PM at each step we re-obtain the decomposition of $\operatorname{Gr}(1,3)^{\operatorname{tnn}}$ in positive subspaces. For example in this case the positive part of the projective toric variety, the interior part of the central diagram in $(2.7)$, describes $\mathbb{R P}_{>0}^{2}$. The boundaries are added by eliminating some of the corners of the diagram and this corresponds to a reduction on the (positive) facets. The first elimination indeed gives three lines that correspond to the 
three $\mathbb{R P}_{>0}^{1}$. The last step identifies the three single coordinates.

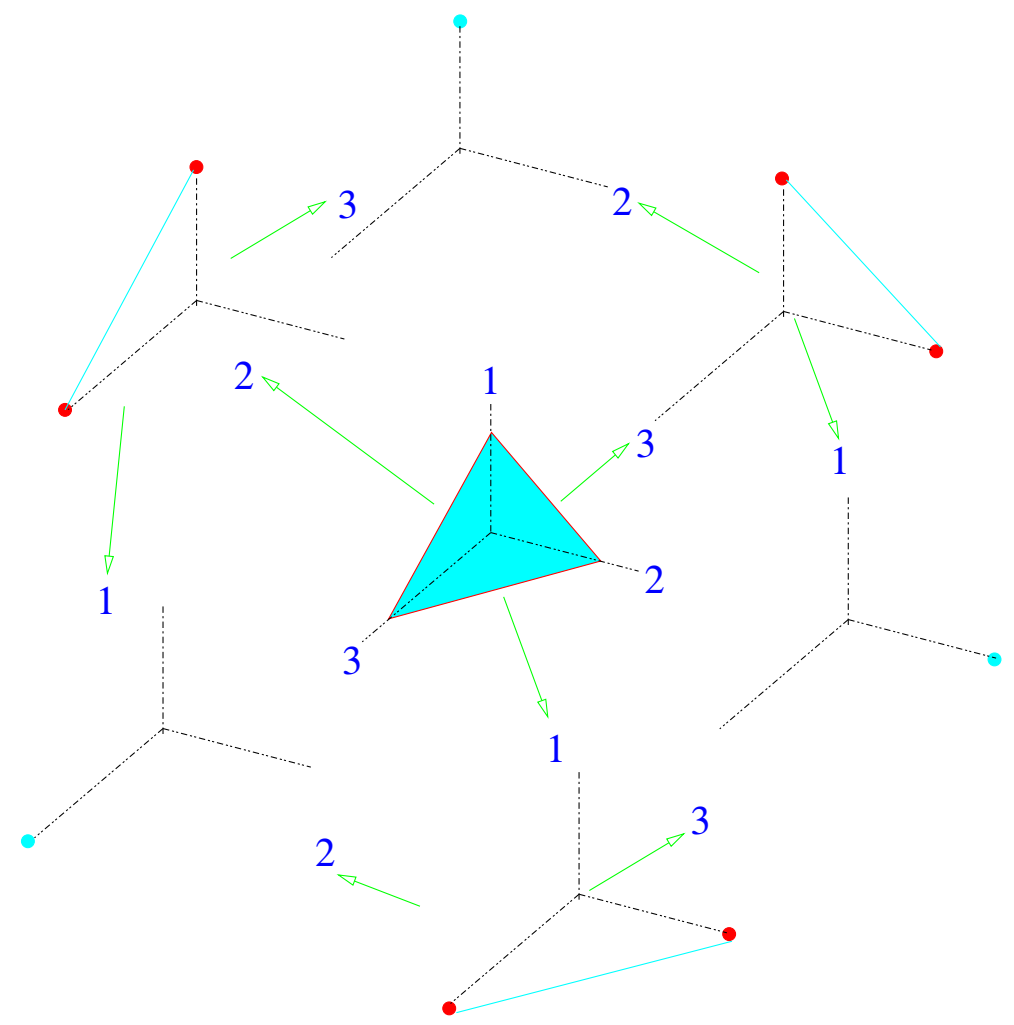

Observe that every reduction corresponds to the elimination of an edge in the diagram, and if more than one PM must be eliminated at the same time one has to erase the edges common to these PM. In this case every edge is associated to a single PM and the situation is simpler.

At every step the elimination of a PM sets to zero the corresponding coordinate. In (2.7) we marked in cyan the interior (positive) part of each toric diagram. The final step of this construction corresponds to the single points that identify each PM.

Summarizing, in the toric picture we started with $\mathbb{R P}_{>0}^{2}$ and we obtain its cell decomposition in positive subspaces by projecting on the facets of the diagram, reproducing exactly the cell decomposition of $G r(1,3)^{t n n}$.

\subsection{The box diagram}

In the previous example the on-shell diagram had no internal faces. In this subsection we want to study a slightly more complicate case, with one internal face. The diagram is shown in $(2.8)$

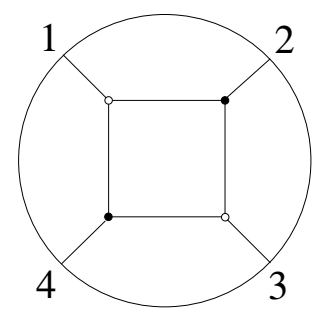


There are two novelties compared to the previous example: first the PMs are not associated to single edges but to collections of edges; second some PMs differ by internal faces and they are associated to the same projective coordinate $p_{I}$.

The on-shell diagram in (2.8) is the box diagram, representing the tree level scattering of four particles, two with positive and two with negative helicity in the planar limit, another example of a tree level MHV amplitude. In the formulation of [26] this on-shell diagram is associated to $\operatorname{Gr}(2,4)^{t n n}$, the non negative part of the space on two-planes in $\mathbb{R}^{4}$. The standard algebraic geometric description of this space is provided, as previously explained, by the Plücker embedding, that in this case is simply a quadratic equation among the $p_{I}{ }^{5}$ coordinates of $\mathbb{R P}_{\geq 0}^{5}$ :

$$
p_{1} p_{6}=p_{2} p_{3}+p_{4} p_{5}
$$

Let us now try to show how the underlining toric geometry associated to the box diagram in (2.8) maps to $G r(2,4)^{t n n}$ and hence it relates to the renewed understanding of scattering amplitudes.

The box diagram has seven PMs, $\sigma_{i}$, as shown in (2.10).
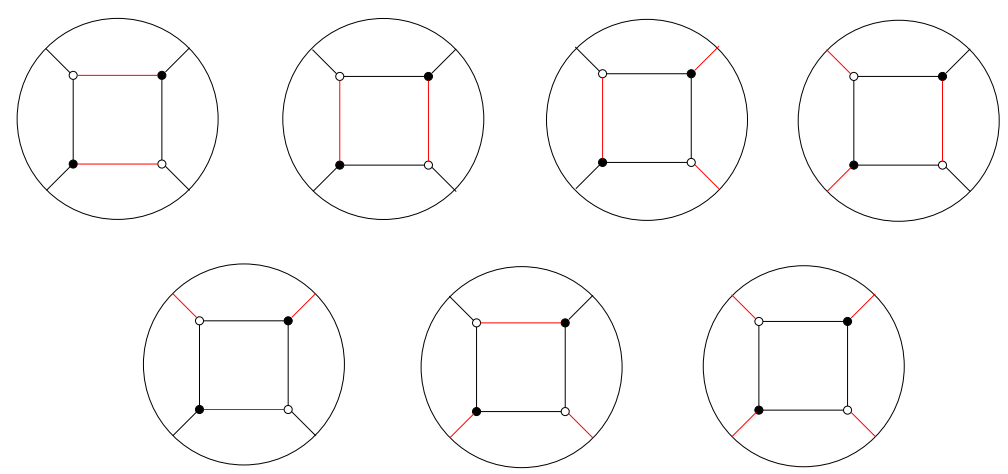

In this case the $\sigma_{i}$ are not free, but they satisfy two relations:

$$
\sigma_{1}+\sigma_{7}=\sigma_{5}+\sigma_{6} \quad \sigma_{2}+\sigma_{7}=\sigma_{3}+\sigma_{4}
$$

As previously explained we can associate a vector $v_{\sigma_{i}}$ to every PM and obtain the polytope in $\mathbb{R}^{5}$ described by these vectors by solving the two equations (2.11) in terms of five vectors, for example $v_{\sigma_{1}}, v_{\sigma_{2}}, v_{\sigma_{3}}, v_{\sigma_{4}}, v_{\sigma_{5}}$. The convex hull described by these seven vectors is the matching polytope of the box diagram and it can be parameterized as follow:

$$
\begin{array}{lll}
v_{\sigma_{1}}=(1,0,0,0,0), & v_{\sigma_{2}}=(0,1,0,0,0), & v_{\sigma_{3}}=(0,0,1,0,0), \\
v_{\sigma_{5}}=(0,0,0,0,1), & v_{\sigma_{6}}=(1,-1,1,1,-1), & v_{\sigma_{7}}=(0,-1,1,1,0)
\end{array}
$$

The toric variety described by this polytope is obtained by associating to every vector $v_{\sigma_{i}}$ a coordinate $\pi_{i}$ in $\mathbb{R P}_{>0}^{6}$, by taking the exponential of the relations in (2.11) and by

\footnotetext{
${ }^{5}$ In the following $I$ will be associated to a specific subset of the $n$ external edges of the on-shell diagram. In this section for simplicity, with a clear abuse of notation, we will use $I$ as the number classifying the set of external edges $I$. We hope that the reader will not be confused.
} 
interpreting them as multiplicative relations among the $\pi_{i}$, and then by taking the closure of this map. We obtain in this way the algebraic equations for the projective toric variety associated to the box diagram. The final result is a toric variety described by two quadrics in $\mathbb{R P}_{\geq 0}^{6}$ :

$$
\pi_{1} \pi_{7}=\pi_{5} \pi_{6} \quad \pi_{2} \pi_{7}=\pi_{3} \pi_{4}
$$

Maybe it worthwhile to underline, as we will explain more in details in the following sections, that the toric variety described by the relations (2.13) is not the Master Space introduced in [48], and further discussed in the case of bipartite graphs on disks in [34], even if the matching polytope in (2.12) is the same. The procedure to associate the coordinates and relations to the polytope used here is indeed different from the one used in the case of the Master Space.

We would like now to illustrate how the two four dimensional varieties in (2.9) and (2.13) are related and hence how the toric variety in (2.13) encodes the information of the four particles scattering process. The map from the toric variety to the Grassmannian, and hence to the scattering amplitude, is given by the equation (2.4) that we introduced in the previous section. This equation essentially boils down to a linear map that relates some combinations of the coordinates of the embedding space $\mathbb{R} \mathbb{P}_{\geq 0}^{6}$ of the toric variety, to the coordinates of $\mathbb{R P}_{\geq 0}^{5}$ : the standard Plücker embedding space for $\operatorname{Gr}(2,4)^{t n n}$. This map will become much more clear when we will explain it in detail in the following sections. For the time being the important observation is that the first two PMs: $\sigma_{1}$ and $\sigma_{2}$, in (2.10), differ by an internal face and hence, according to (2.4), their sum should be associated to a single $p_{I}$. The final map is:

$$
p_{1}=\pi_{1}+\pi_{2}, \quad p_{2}=\pi_{3}, \quad p_{3}=\pi_{4}, \quad p_{4}=\pi_{5}, \quad p_{5}=\pi_{6}, \quad p_{6}=\pi_{7}
$$

These $p_{I}$ coordinates defined in this way satisfy then the Plücker embedding equation (2.9) for $\operatorname{Gr}(2,4)^{t n n}$, once the toric $\pi_{i}$ coordinates are restricted on the toric variety $(2.13)$.

In this case the toric diagram lives on a five dimensional lattice and we cannot give a pictorial description of it. However, as before, we can decompose this totally non negative space in totally positive subspaces associated to (sub)cells in $\operatorname{Gr}(2,4)^{t n n}$ and hence to the singularities required to reconstruct the scattering amplitude. The codimension one cells in $\operatorname{Gr}(2,4)^{t n n}$ are associated to some facets of dimension 4 in the matching polytope in (2.12). One can end up on those facets by erasing some of the PMs. We will carefully redo all this procedure in the next sections, once all the required technology will be introduced. The

\begin{tabular}{|c|c|c|c|}
\hline Vanishing $\pi_{I}$ & Surviving relation & Vanishing $p_{I}$ & Surviving relation \\
\hline$\pi_{1}=\pi_{5}=0$ & $\pi_{2} \pi_{7}=\pi_{3} \pi_{4}$ & $p_{4}=0$ & $p_{1} p_{6}=p_{2} p_{3}$ \\
\hline$\pi_{1}=\pi_{6}=0$ & $\pi_{2} \pi_{7}=\pi_{3} \pi_{4}$ & $p_{5}=0$ & $p_{1} p_{6}=p_{2} p_{3}$ \\
\hline$\pi_{2}=\pi_{3}=0$ & $\pi_{1} \pi_{7}=\pi_{5} \pi_{6}$ & $p_{2}=0$ & $p_{1} p_{6}=p_{4} p_{5}$ \\
\hline$\pi_{2}=\pi_{4}=0$ & $\pi_{1} \pi_{7}=\pi_{5} \pi_{6}$ & $p_{3}=0$ & $p_{1} p_{6}=p_{4} p_{5}$ \\
\hline
\end{tabular}
first step of this reduction is

where on the right we present the four codimension one cells of $\operatorname{Gr}(2,4)^{t n n}$, and on the left the corresponding toric codimension one subspaces of the associated toric variety. Every 
choice corresponds to a different singularity in the amplitude. In (2.15) we identify the vanishing PMs, the vanishing coordinates $p$ and the relations among the other surviving coordinates. It is important to remember that the surviving $p$ and $\pi$ coordinates are constrained to have only positive and non zero values. The toric sub dimensional varieties and the cells are isomorphic. Further reductions to higher codimensional cells and facets are possible. For example from the three dimensional cell and facet in in the first line of (2.15) one can reduce to the sub-facets by imposing either $\pi_{2}=\pi_{3}=0$, or $\pi_{2}=\pi_{4}=0$, or $\pi_{6}=0$. This corresponds to set to zero other $p$ coordinates and hence go to subcells in the cell decomposition.

By looking at all the possibilities we can summarize the first two steps in the cells/facets decomposition in the following table

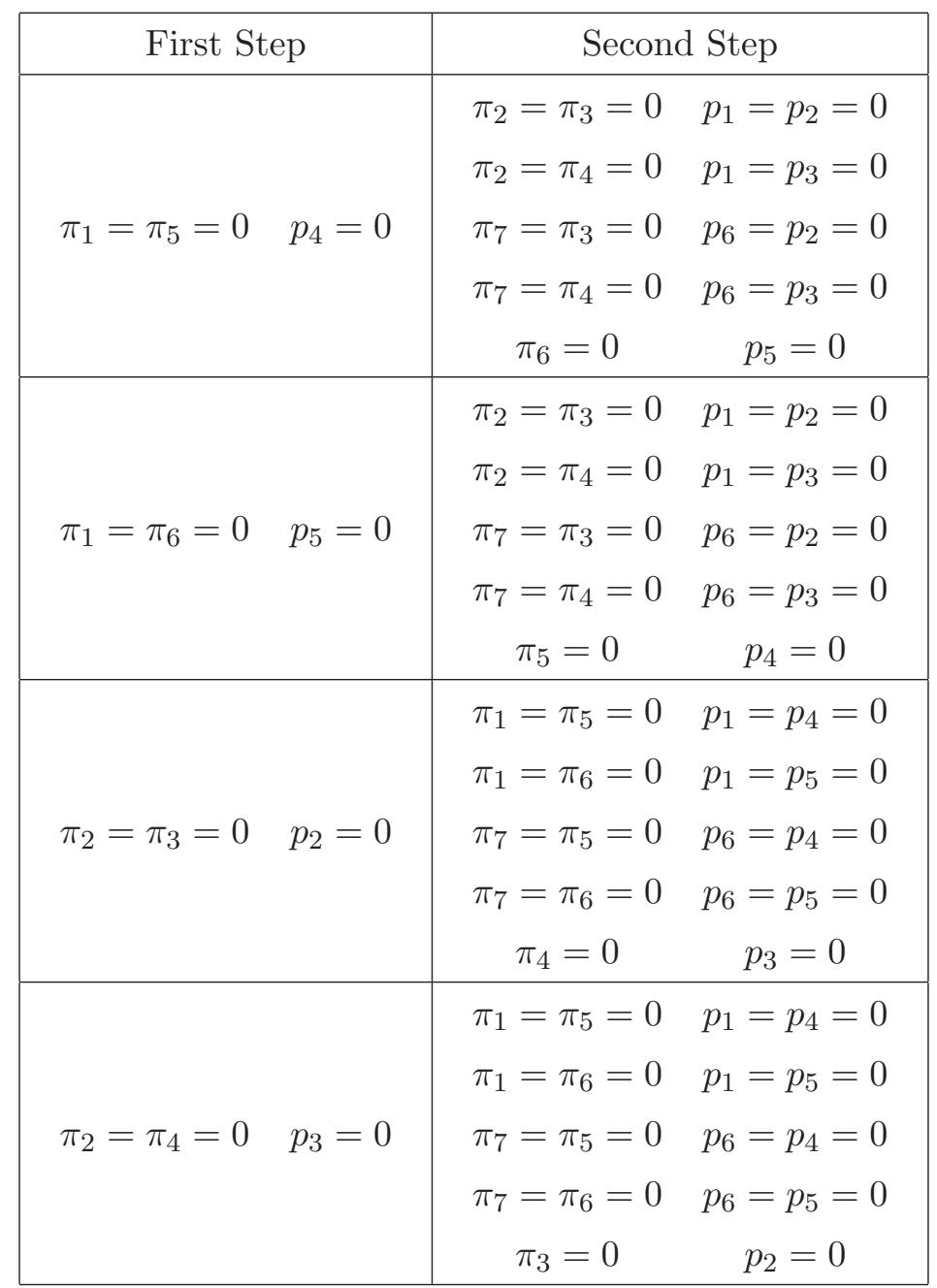

At the second step we can identify twenty possibilities but just ten of them are different (in the other cases we are setting to zero the same set of $\pi$ coordinates, but in a different order). We can then resume the two dimensional cells of $G r(2,4)^{\operatorname{tnn}}$ and the associated two dimensional toric varieties as in the table 


\begin{tabular}{|c|c|c|}
\hline$p_{I}=0$ & $\pi_{I}=0$ & relations \\
\hline$(1,2,4)$ & $(1,2,3,5)$ & \\
$(1,3,4)$ & $(1,2,4,5)$ & \\
$(1,2,5)$ & $(1,2,3,6)$ & \\
$(1,3,5)$ & $(1,2,4,6)$ & \\
$(2,4,6)$ & $(1,3,5,7)$ or $(2,3,5,7)$ & \\
$(3,4,6)$ & $(1,4,5,7)$ or $(2,4,5,7)$ & \\
$(2,5,6)$ & $(1,3,6,7)$ or $(2,3,6,7)$ & \\
$(3,5,6)$ & $(1,4,6,7)$ or $(2,4,6,7)$ & \\
$(2,3)$ & $(2,3,4)$ & $p_{1} p_{6}=p_{4} p_{5}$ \\
$(4,5)$ & $(1,5,6)$ & $p_{1} p_{6}=p_{2} p_{3}$ \\
\hline
\end{tabular}

Let us observe that in the first four lines the projection of the diagram identifies a single subspace. In the next four cases there are two possible reductions of the toric diagram that identify the same subspace. This is a common feature: by projecting on the faces of the diagram we end up on a sub cell of the Grassmannian. But different projections can identify the same cell, if they are obtained sending to zero $\pi$ associated to PMs differing by internal faces. This is related to the fact that the map between the toric variety and the Grassmannian is not in general an isomorphism. By iterating this procedure one obtains the other lower dimensional cells. The process finishes when one has only one non vanishing $p$.

\section{Bipartite diagrams, polytopes and toric geometry}

Until this point we based our discussion on an intuitive understanding. In the rest of the paper we will go through in detail in the relation between toric geometry and the top form in (2.2) associated to the scattering amplitude. In particular we will deeply investigate the connection between the bipartite graphs on the disk and the decomposition of the totally non negative Grassmannian in positive subspaces, or cells. In addition we will analyze the detailed relation between bipartite graphs on a disk and toric geometry. We will then reformulate a relation between the toric and the Grassmannian picture and, elaborating on previous results, we will provide a toric geometrical understanding for the top form in (2.2) associated to scattering amplitude.

We start this analysis by reviewing some basic definitions about the bipartite graphs on the disk.

A graph is a set of vertices connected by edges. If every edge connects a black and a white vertex the graph is called bipartite. A graph on the disk has two types of edges, internal and external edges. The internal edges connect two vertices, while the external edges connect a node to the boundary of the disk. The external edges do not intersect at the boundary.

Bipartite graphs have been extensively studied in the analysis of D3 branes probing toric $\mathrm{CY}_{3}$ singularities in the $\mathrm{AdS}_{5} / \mathrm{CFT}_{4}$ correspondence [28-33]. The difference with the 
case that we discuss here is that the graphs do not live on a torus but on a disk, where there are also external edges.

There is a useful quantity in the analysis of bipartite diagrams both on the disk and on the torus, that we will often use in this paper. This is the notion of perfect matchings (PMs). A PM on a bipartite diagram on a torus is a set of edges without common vertices that covers all the vertices of the graphs. Every edge in a PM connects a white to a black node. In the case of the disk there is a similar definition of PMs. The difference is that the external edges cover a single vertex. One can see an example of PMs on the disk in (2.10).

Closely related to the notion of PMs one can define the perfect orientations, POs. This definition does not have any particular role in the case of the torus but it becomes important in this setup. A PO is defined by assigning an arrow to every edge in the graph such that

- Every black vertex is incident to just one edge directed away from the vertex.

- Every white vertex is incident to just one edge directed toward the vertex.

As shown in $[27,34,41]$ there is a 1-1 correspondence between PM and PO. Given a PO the associated PM is composed by the set of edges directed from the black to the white nodes in the PO. On the other hand, given a PM, one can construct a PO by assigning arrows from black to white nodes to the internal edges in the PM and arrows from white to black nodes to the internal edges not in the PM. For the external edges one assigns an ingoing (outgoing) arrow to the edges in the PM ending on a white (black) node and an ingoing (outgoing) arrow to the edges not in the PM ending on a black (white) node. One can observe an example of the correspondence between the PMs and the POs in (6.3).

\subsection{Polytopes}

Similarly to the case of the torus the PMs can be used to define a set of polytopes associated to a bipartite diagram. These polytopes are used to associate toric varieties to these diagrams. There are two polytopes naturally associated to a bipartite graph, the matching and the matroid polytope.

The matching polytope. The first polytope that is usually associated to a bipartite diagram is the so called matching polytope. The matching polytope can be defined by using the $s$ linear relations among the $c$ perfect matchings. It is indeed the convex hull described by the $c$ vectors of length $c-s$ that satisfy the $s$ linear constraints. It turns out that the number of faces of the bipartite graph $G$ is equal to $c-s$, and the matching polytope lives in a space of $G$ dimensions. ${ }^{6}$

In its original definition [41] the matching polytope was defined as follows. Every PM $\sigma_{i}, i=1, \ldots, c$, is associated to a set of edges. One associates a $P_{c, E}$ matrix to the bipartite diagram, where $c$ labels the PMs and $E$ the edges. The element $P_{c_{i}, E_{j}}$ of the matrix $P$ is 1 if the edge $E_{j}$ belongs to the $c_{i}$-th PM, 0 otherwise.

\footnotetext{
${ }^{6}$ There is an extra relation among the vectors forming the polytope. Indeed as we will show in section B the vectors are all coplanar and the polytope is actually a $G-1$ dimensional object.
} 
The two definitions of matching polytope are equivalent because the PMs are not all independent, and one can reduce the $P$ matrix by the relations between the PMs. There are $G$ remaining components. This polytope is usually referred as the toric diagram of the Master Space if the bipartite diagram is drawn on a torus [48]. This terminology was used also in [34] in the field theory interpretation of the bipartite diagrams on the disk. Here we prefer to keep on referring to the matching polytope also for the projection of the matching polytope defined in [41].

The matroid polytope. A second natural polytope that one can define on the bipartite diagram is the matroid polytope. In appendix A.1 we will review the basic aspects of the matroids. Here we just give a computative definition of the matroid polytope.

The matroid polytope is obtained from the matching polytope, by considering all the internal faces $G_{\text {int }}$ and by adding extra linear relations. These relations are the identification of PMs that differ by an internal face. This procedure projects the $G$ dimensional matching polytope on a $G-G_{\text {int }}$ dimensional polytope called the matroid polytope. ${ }^{7}$

In the language of the POs one can define a matroid polytope as follows. First one starts by identifying the POs associated to the same source set. A source is the external label associated to an external line having an arrow pointing on the interior of the graph. Then one associates a $s \times E$ matrix $Q$ to the bipartite diagram where $s$ is the number of different sources and $E$ is the number of external edges. The entry of the matrix $Q_{s_{i}, E_{j}}$ is 1 if, after the identification, the $E_{j}$-th edge is in the $s_{i}$-th PO, 0 otherwise. As shown in [37] this construction is equivalent to the projection of the master space on another polytope, usually called the toric diagram of the moduli space in the brane tiling interpretation. The projection is usually done by modding the master space by the internal face variables. Since POs associated to the same source set differ by internal faces, modding the matching polytope by them reproduces the projection on the matroid polytope. In the rest of the paper we will refer to the toric diagram of the moduli space as the matroid polytope.

\subsubsection{A remark on polytopes and toric geometry}

At this point of the discussion we want to distinguish the relevant aspects of the toric geometry associated to the bipartite field theories (BFTs) defined in [34] and the one relevant for the study of the scattering amplitudes.

As we have just reviewed the matching and the matroid polytopes correspond to the toric diagram of the master and of the moduli space in the field theoretical interpretation (in the case of the torus) and this interpretation can be extended to the case of the disk. It was done in [34], where it was observed that these theories are not generically SCFTs, and the external edges are not dynamical fields. Another interpretation of these BFTs was given in $[35,36]$ and the relation between the two interpretations was explained in [38].

In the case of the BFTs one associates to every vector a coordinate in $\mathbb{C}^{c}$ and the master space is an affine variety obtained from the symplectic quotient with the linear

\footnotetext{
${ }^{7}$ As in the case of the matching polytope also the vectors that form the matroid polytope are coplanar and the actual dimension of the polytope is reduced by one.
} 
relations between the PMs. The moduli space is obtained with an extra quotient by the internal faces.

In the case of the scattering amplitudes the associated toric variety is projective and non affine and its construction will be explained in section 5 .

It is maybe worthwhile to state that even if the polytopes in the BFT and in the scattering amplitude are the same, the associated toric varieties are different. Indeed in the first case the linear relations among the PMs are implemented as algebraic polynomial equations on the $\pi_{i}$, while in the second case they are implemented as symplectic quotient relations.

\section{Coordinates on $\operatorname{Gr}(k, n)^{t n n}$ from bipartite graph}

In this section we first review the parameterization of the totally non negative Grassmannian in terms of ratios of Plücker coordinates as discussed in [47]. We refer the reader to appendix A.1 for the basic definitions of the Plücker embedding and the Grassmannian. Then we show that these coordinates become simpler if expressed in terms of the PMs.

The connection between the bipartite graphs and $\operatorname{Gr}(k, n)^{t n n}$ is obtained through the boundary measurement. We review the mathematical aspects of this connection and the definition of the measurement in the appendix A.2, while here we try to keep the discussion more simple and intuitive.

The boundary measurement is a map that assigns a subset in $\operatorname{Gr}(k, n)^{t n n}$ to a bipartite graph. Explicitly one fixes a perfect orientation. This defines a set of sources and a set of sinks in the set of the external edges. A source is an external edge directed inside the graph in the PO, while a sink is directed through the boundary, as in (4.5). For every PO one has $k$ sources and $n-k$ sinks. From this definition of sources and sinks one can define a $k \times n$ matrix $A$. The $k$ row entries correspond to the sources and the $n$ columns are all the possible external edges.

It is possible to assign a positive real variable to every edge and then express the entries in $A$ as the product of the edge variables connecting the external edge in the $i$-th row of $A$ with the external edge in the $j$-th column. Every minor of $A$ is a Plücker coordinate of $\operatorname{Gr}(k, n)^{t n n}$. The final matrix $A$ will be composed by a $k \times k$ identity matrix corresponding to the paths from the sources to the sources, and a $k \times n-k$ matrix corresponding to the paths from the sources to the sinks. If the bipartite diagram is associated to the full Grassmannian the $k \times n-k$ sub matrix have all non zero positive entries, If instead the bipartite diagram is associated to a sub space of the Grassmannian then some of the entries of the $k \times n-k$ matrix are zero and they provide the specification of the cell. This procedure gives a set of local coordinates of a patch of $G r(k, n)^{t n n}$. The peculiar patch is singled out by the determinant of the $k \times k$ identity matrix that fixes to 1 the value of one to the Plücker coordinates associated to the selected PO. There are $\left(\begin{array}{l}n \\ k\end{array}\right)$ of these patches. One can glue the patches associated to each PO, obtaining a global coordinatization of $\operatorname{Gr}(k, n)^{t n n}$ or of the closure of the cell associated to the bipartite graph. 


\subsection{Coordinates from the flows}

An explicit algorithm to obtain the local coordinates of $G r(k, n)^{t n n}$ from a bipartite graph was discussed in [47]. This procedure provide the expression for the ratios of the $\left(\begin{array}{l}n \\ k\end{array}\right)-1$ Plücker coordinates over the Plücker coordinate associate to the selected PO.

First of all one has to define the notion of flow. By fixing the source set $I_{\mathcal{O}}$ and another set of external vertices $J$ with the same length as $I_{\mathcal{O}}$ a flow from $I_{\mathcal{O}}$ to $J$ is a collection of disjoint and self avoiding paths from $I_{O}-I_{O} \cap J$ to $J-I_{O} \cap J$. A flow from $I_{O}$ to $I_{O}$ is called conservative. One can then construct a ratio between the edge variables in every flow by putting in the numerator the edges directed from the white to the black vertices and in the flow and in the denominator the edges from black to white. This ratio is defined as weight of the flow, $w(F)$. From now on we will call $\Delta_{J}(A)$ the ratio of the $k$-minor associated to $J$ and the $k$-minor associated to $I$ (this last one is equal to one in this patch): the set of $k$ sources provided by the selected PO. A a set of local coordinates for this patch is given by:

$$
\Delta_{J}(A)=\frac{\sum_{F: I_{O} \rightarrow J} w(F)}{\sum_{F^{\prime}: I_{O} \rightarrow I_{O}} w\left(F^{\prime}\right)}=\frac{p_{J}}{p_{I}}
$$

This is an explicit realization of the connection between $\operatorname{Gr}(k, n)^{t n n}$ and a bipartite graph. $\Delta_{J}(A)$ are well defined coordinates in the patch associated to the $I$ sources, and they are equal to the Plücker coordinates $p_{J}$ in this patch. However they only provide a set of local coordinates in this patch and the Plücker coordinates $p_{J}$ are in general different from the $\Delta_{J}(A)$ outside this patch. The $p_{J}$ provide instead a set of global coordinates, somehow similar to the homogeneous coordinates of the projective space. ${ }^{8}$

\subsection{Coordinates from the PMs}

In this section we explain in detail the simple map (2.4), already introduced in section 2, between the Plücker coordinates $p_{J}$ and the $\pi_{i}$ toric embedding coordinates associated to the PMs, and we provide an explicit proof for it. This map provides an explicit relation between the embedding coordinates of the toric variety associated to a given on-shell diagram and the Plücker embedding coordinates of the closure, in the totally non negative Grassmannian, of the cell associated to the same on-shell diagram. This is a global map, in the sense that it is defined on the embedding coordinates and not only on the local coordinates on a patch, and provides a way to reformulate the scattering amplitudes in terms of toric varieties.

In $G r(k, n)^{t n n}$ given a source set of $k$ elements $I=\left(i_{1}, \ldots, i_{k}\right)$ and another set $J=$ $\left(j_{1}, \ldots, j_{k}\right)$ one can associate to $I$ a set of PM coordinates labelled by $\pi_{I}^{\gamma}$ and to $J$ a set of PM coordinates labelled by $\pi_{J}^{\beta}$.

This represents a new notation associated to the PMs coordinates $\pi_{i}$. This new notation has two indexes, a capital latin index that denotes the source set of the associated $\mathrm{PO}$, and a greek index that labels the degeneration (multiple PMs can be associated to the same source set). The PMs associated to the same source set differ by internal loops

\footnotetext{
${ }^{8}$ Note that the $p_{I}$ that we are defining in this paper differ from the $p_{I}$ of $[41,47]$ since they are global coordinates here and local coordinates there. In other words we are not defining $p_{J}=\sum_{F: I_{O} \rightarrow J} w(F)$.
} 
in the POs. We hope that this double notation for the same quantity does not generate confusion in the reader.

We are going to prove that the Plücker coordinates $p_{J}$ and the toric coordinates $\pi_{J}^{\beta}$ are simply related by a linear map:

$$
p_{J}=\sum_{\beta} \pi_{J}^{\beta}
$$

This result is proven by showing that this formulation is equivalent to the one in equation (4.1) and proven in [47], in terms of flows and conservative flows. We will first show that the equation (4.1) can be equivalently written in terms of PMs coordinates as:

$$
\Delta_{J}=\frac{p_{J}}{p_{I}}=\frac{\sum_{\beta} \pi_{J}^{\beta}}{\sum_{\gamma} \pi_{I}^{\gamma}}
$$

Once this local expression is obtained it is easy to derive the global relation (4.2) by simply considering that the $p_{J}$ and the $\pi_{J}^{\beta}$ coordinates define a point in the Grassmannian and in the toric variety only modulo a multiplication by a common factor. We can then erase the denominators and find the equation (4.2). More formally one can consider that (4.3) is a local map valid on the patch in the closure of the cell in the totally non negative Grassmannian such that $p_{I}$ is different from zero, and in the patch in the totally non negative toric variety such that $\sum_{\gamma} \pi_{I}^{\gamma}$ is non vanishing. It is then possible to glue the map defined on the $I$ patch with the one defined on the $K$ patch by using the transition functions:

$$
f_{I K}=\frac{p_{I}}{p_{K}}=\frac{\sum_{\beta} \pi_{I}^{\beta}}{\sum_{\gamma} \pi_{K}^{\gamma}}
$$

By iterating this procedure in every patch one obtains the global map (4.2).

Let us start with the proof of (4.3). In [47] each coordinate is specified by a set $J$, corresponding to the source set. By fixing a set $I$ of sources the numerator of (4.1) is identified with the non overlapping paths (referred as the flows) from $I-\{I \cap J\}$ to $J-\{I \bigcap J\}$. The denominator is given by the conservative flows. A flow is conservative if it is obtained from a fixed perfect orientation $\mathrm{PO}_{I}^{\alpha}$ by considering all the possible disconnected loops consistent with the choice of arrows in $\mathrm{PO}_{I}^{\alpha}$. Such a $\mathrm{PO}$ will be referred as reference $\mathrm{PO}$ in the rest of the paper.

The coordinates in $G r(k, n)^{t n n}$ are expressed in terms of the paths in the bipartite graph. We want to express them in terms of the PM coordinates $\pi$. This can be done by observing that the ratio of two PM coordinates $\pi_{i}$ and $\pi_{j}$ is a path in the bipartite diagram. The orientation of the these paths is fixed as follows. One starts by assigning an orientation to the edges in each PM (for example from black to white). Then in the ratio of two PM coordinates (the ratio of the edges that compose the PM themselves) one keeps the black to white orientation in the numerator and reverse the orientation in the denominator. In this way one obtains an oriented path in the bipartite diagram. In terms of the edges this is a ratio of edges, the ones from black to white in the numerator and the ones from white to black in the denominator. 
There are two possible types of paths in the disk, the closed paths that surround an internal face and the open paths from the boundary to the boundary. Note that a ratio of PMs can also be the composition of disconnected open and/or closed paths.

The description of the paths is simplified once one expresses them in terms of the POs. Indeed for every choice of reference $\mathrm{PM}_{I}^{\alpha}$ the possible open and closed paths on the disk are identified by the direction of the arrows in $\mathrm{PO}_{I}^{\alpha}$. The ratio of the $\mathrm{PM}$ coordinates associated to the source set $J, \pi_{J}^{\beta}$ (with $\beta$ running over the possible multiplicity, different PMs with sharing the same source set) by the reference coordinate $\pi_{I}^{\alpha}$ (where $\alpha$ is fixed) is obtained as follows. We keep fixed the orientation of the arrows in $\mathrm{PO}_{I}^{\alpha}$ and reverse the orientation of the arrows of $\mathrm{PO}_{J}^{\beta}$ for each $\beta$. In terms of the edges we divide the product of edges in $\mathrm{PM}_{J}^{\beta}$ by the edges in $\mathrm{PM}_{I}^{\alpha}$. This generates a path, either open, either closed or a combination of both, on the disk. This is exactly the same construction described above in terms of the PMs.

Formula (4.3) is equivalent to fix the source set $I$ and obtain the $J$-th coordinate as the sum of all the possible non overlapping loops from $I-\{I \cap J\}$ to $J-\{I \cap J\}$ divided by the sum of the disconnected closed ones in $\mathrm{PO}_{I}^{\alpha}$.

We start by studying the case with $I \neq J$. The path connects an even number (at least two) of points on the boundary of the disk. We will now prove that this is a flow from $I-\{I \cap J\}$ to $J-\{I \cap J\}$. This is shown in two steps.

\section{Sources and sinks}

First one has to find the source and the sink set of the flow. There are two possible types of edges in the source set of the flow. The first type of source edges are the incoming edges of $\mathrm{PO}_{I}^{\alpha}$ highlighted in red in $\mathrm{PM}_{I}^{\alpha}$ and not highlighted in red in any $\mathrm{PM}_{J}^{\beta}$. The second type of source edges in the flow are the source edges of $\mathrm{PO}_{I}^{\alpha}$ not highlighted in red in $\mathrm{PM}_{I}^{\alpha}$ but highlighted in red in every $\mathrm{PM}_{J}^{\beta}$. An analogous discussion holds for the sink set. This description of the source and sink set of the flow is equivalent to the definition of [47]: the sources of the flow are in $I-\{I \cap J\}$ and the sinks are in $J-\{I \cap J\}$. For example we consider $\mathrm{PO}_{3}$ as the reference PO in $\operatorname{Gr}(2,4)^{t n n}$ and we look for the Plücker coordinate associated to $\mathrm{PO}_{7}$

$$
\begin{aligned}
& \text { Perfect matching } \\
& \text { and } \\
& \text { perfect orientation }
\end{aligned}
$$

Parametrization

Source set

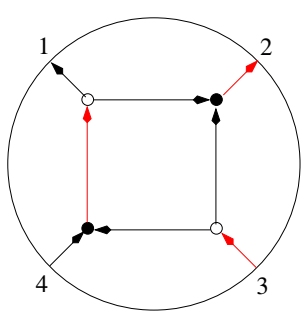

$\pi_{3}$

$\{3,4\}$

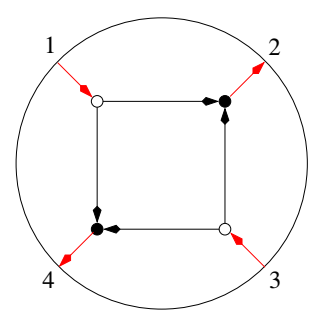

$\pi_{7}$

$\{1,3\}$ 
The source set of the flow is obtained from the rules given above

- The only edge in the source set of the reference perfect orientation and highlighted in $\mathrm{PM}_{3}$ is the external edge connected to the external vertex labelled by 3. This edge is also in $\mathrm{PM}_{7}$ and it cannot be in the flow.

- The only edge in the source set of the reference perfect orientation and not highlighted in $\mathrm{PM}_{3}$ is the external edge connected to the external vertex labelled by 4 . This edge is also in $\mathrm{PM}_{7}$ and it is the source set of the flow.

One can find that in the same way the sink set of the flow is given by the external edge connected to 3 . The final flow is the same path obtained with the prescription of $[47]$.

\section{Non overlapping paths}

Since the path is given by a ratio of PMs every vertex is crossed either zero or two times by each path. This shows that every path has no overlap.

The denominator in (4.3) is obtained by considering all the possible non overlapping closed loops in the reference perfect orientation $\mathrm{PO}_{I}^{\alpha}$ and summing over them. This is equivalent to consider all the possible $\mathrm{PO}_{I}^{\gamma}$ with $\gamma \neq \alpha$. In terms of the $\mathrm{PM}$ coordinates the denominator is

$$
\frac{\pi_{I}^{\alpha}+\sum_{I} \pi_{I}^{\gamma}}{\pi_{I}^{\alpha}}
$$

The first term corresponds to the trivial path, the absence of loops.

The other terms are obtained from the reference PO labelled by $\alpha$ by inverting the orientation of some of the internal loops. This procedure generates a new PO labelled by $\gamma$, with the same sink and source set of the reference PO. Every PM labelled by $\gamma$ with the same source set of the PM labelled by $\alpha$ is obtained in this way: one starts from the reference $\alpha$ and inverts one or more disconnected closed loops, obtaining a new perfect orientation with the same source set $I$. At the level of the coordinates for every $\gamma$ the ratio $\frac{\pi_{I}^{\gamma}}{\pi_{I}^{\alpha}}$ corresponds to one of the possible disconnected loops in $\mathrm{PO}_{I}^{\alpha}$. All the loops are obtained by this prescription because, by fixing $I$, all the possible choices of internal arrows differ by a closed loops. By starting from one perfect orientation and by inverting all the (possibily disconnected) internal loops all the POs corresponding to the same source set $I$ are obtained. 
We give an explicit example of this construction in (4.7)

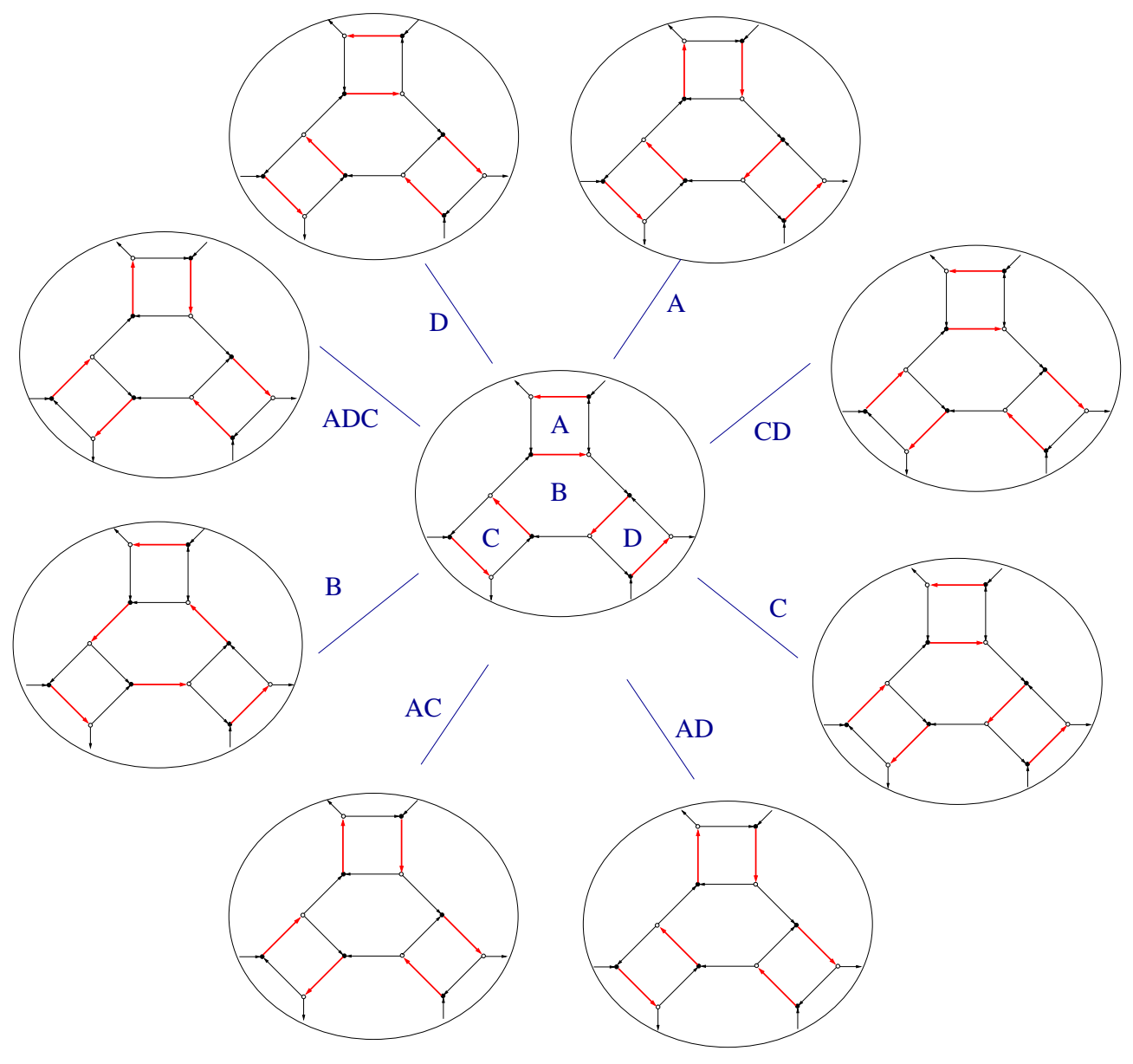

A subcell of $\operatorname{Gr}(3,6)^{t n n}$

This is the case of a subcell of $\operatorname{Gr}(3,6)^{t n n}$. All the possible PMs sharing the same source set of the reference PM are obtained as follows. One fixes the external sources and one PM corresponding to that source. This also sets the reference PO. This PO may have some internal loops. For example here we label these loops as $A, B, C$ and $D^{9}$ in (4.7). The other PMs are found by reversing the orientation of all the combinations of the internal loops. In this case for example we have 8 possibilities as shown in (4.7). Every link connecting two different POs labels the loops that are reversed. The ratio of the first PM and one of the others specifies a disconnected set of loops. In terms of coordinates we have

$$
1+\sum_{\gamma \neq \alpha} \frac{\pi_{I}^{\gamma}}{\pi_{I}^{\alpha}}=1+A+B+C+D+A D+A C+C D+A C D
$$

This is the same denominator obtained by applying the prescription of [47].

\footnotetext{
${ }^{9}$ In this case we identify with the capital latin letters $A, B, C$ and $D$ also the coordinates associated to the loop, namely the ratio of the edges from black to white divided by the edges from white to black, such that the loop is counterclockwise.
} 
To complete the proof of (4.3) we must discuss the case of $I=J$. In this case the numerator and the denominator coincide and one obtains $\Delta_{I}=1$, that fixes the patch in this local coordinate system.

\section{Toric geometry and cell decomposition}

In this section, elaborating on recent results in the mathematical and physical literature, we discuss the relation between the bipartite diagrams on disks and toric varieties, and we explain the cell decomposition of $G r(k, n)^{t n n}$.

A bipartite diagram is associated to a matching polytope $P$ as explained in section 3.1. $P$ is the convex hull of a set of $c$ vectors $v_{\sigma_{i}}$ in $G$ dimensions: namely a $G$ dimensional toric diagram. This toric diagram implements the linear relations among the PMs $\sigma_{i}$ as linear relations among the vectors $v_{\sigma_{i}}$ that define it.

The polytope $P$ defines a projective toric variety as explained in $[49,50]$. The Gvectors $v_{\sigma_{i}}$, with components: $v_{\sigma_{i}}=\left(v_{\sigma_{i}}^{1}, \ldots, v_{\sigma_{i}}^{G}\right)$, define a projective toric variety $X_{P}$ as the closure of the image of the map $\phi:\left(\mathbb{C}^{*}\right)^{G} \rightarrow \mathbb{C P}^{c-1}$

$$
\phi: \mathbf{t}=\left(t_{1}, \ldots, t_{G}\right) \rightarrow\left[\mathbf{t}^{v_{\sigma_{1}}}, \ldots, \mathbf{t}^{v_{\sigma_{c}}}\right]=\left[\pi_{1}, \ldots, \pi_{c}\right]
$$

where $\mathbf{t}^{v_{\sigma_{i}}}=t_{1}^{v_{\sigma_{i}}^{1}} t_{2}^{v_{\sigma_{i}}^{2}} \ldots t_{G}^{v_{\sigma_{i}}^{G}}$

The real part of $X_{P}(\mathbb{R})$ is defined to be the intersection of this variety with $\mathbb{R P}^{c-1}$; the positive part of this real projective variety $X_{P}^{>0}$ is defined to be the image of $\left(\mathbb{R}_{>0}\right)^{G}$ under $\phi$; its non negative part $X_{\bar{P}}^{>0}$ is defined to be the closure of $X_{P}^{>0}$ in $X_{P}(\mathbb{R}) . X_{\bar{P}}^{\geq 0}$ is the main actor in our reinterpretation of scattering amplitudes in terms of toric geometry. Indeed (4.2) provides a map from $X_{\bar{P}}^{\geq 0}$ to $G r(k, n)^{t n n}$, and hence to the scattering amplitudes.

The procedure to obtain $X_{P}$ is somehow intuitively equivalent to interpreting the matching polytope $P$ as the dual toric cone of an associated toric variety. However the variety defined in this way is in general non normal, meaning that the vectors defining $P$ generate the associated cone on the real numbers but not, in general, on the integers. The toric ideal $I_{P}$ of a projective toric variety $X_{P}$ is the homogeneous ideal of all the binomials in $\pi_{i}$ that vanish on the closure of the image of $\phi$.

One very easy example in the polytope in $\mathbb{R}^{3}$ defined by the four vectors $v_{\sigma_{i}}$ satisfying the linear relation:

$$
v_{\sigma_{1}}+v_{\sigma_{4}}=v_{\sigma_{2}}+v_{\sigma_{3}}
$$

In this case the toric ideal is generated by the binomial $\pi_{1} \pi_{4}-\pi_{2} \pi_{3}$, and the non negative real projective toric variety $X_{\bar{P}}^{\geq 0}$ is defined as the zero locus of this binomial, i.e. $\pi_{1} \pi_{4}=$ $\pi_{2} \pi_{3}$, in $\mathbb{R P}_{\geq 0}^{3}$.

The Plücker coordinates $p_{J}$ are assigned to combination of the $\pi_{i}$ as explained in section 4.2. There is a global way to assign the coordinates: one specifies all the possible POs and groups the ones with the same source set. The sources set specifies the Plücker coordinates $p_{J}$, every PO specifies one $\pi_{i}$ : the $p_{J}$ is given as the sum of the $\pi_{i}$ coordinate assigned to POs with the same sources. The constraints on the Plücker coordinates follow 
from the constraints on the $\pi_{i}$ in the toric ideal and they reproduce the usual Plücker relations.

This map from $X_{\bar{P}}^{>0}$ to $G r(k, n)^{t n n}$ is an isomorphism for strictly positive values of the coordinates on the toric variety and on the Grassmannian, while it in general fails to be a one to one map for the full non negative part of the toric variety. However this map allows the description of the cell decomposition of $G r(k, n)^{t n n}$ and hence of the singularities of the integrands for the scattering amplitudes.

The restriction to the positive values of $\pi_{i}$ for the toric variety is isomorphic to the top dimensional cell of the Grassmannian, namely the Grassmannian itself with all the $p_{J}$ strictly positive. The subcells are obtained by deleting the removable edges, defined in section 2, in the associated on-shell diagram. The removal of an edge corresponds to remove the $\mathrm{PM} \sigma_{j}$ containing the edge itself. In the polytope $P$ it implies that we are restricting to the codimension one external sub-polytope described by the vectors $v_{\sigma_{i}}$ that are the ones that are not associated to the $\sigma_{j}$ just removed. Starting from $P$ one can erase the row corresponding to the edge that has been removed and the column that corresponds to the removed PM. By removing one edge on a graph $\mathcal{G}$ one obtains a subgraph $\mathcal{H}$.

The new graph $\mathcal{H}$ is associated to a sub-cell of the Grassmannian [27, 41]. The cell is parameterized as before. One assigns the Plücker coordinates from the POs and the Plücker relations follow from the constraints on the toric ideal of the sub-polytope. By iterating this procedure one obtains the decomposition of $G r(k, n)^{t n n}$ in positive cells. ${ }^{10}$

In $[27,41]$ the details of the positroid cell decomposition of the totally non negative Grassmannian are discussed. Here we report the main results. The closure of each cell is homeomorphic to a closed ball, and the boundary of each cell is homeomorphic to a sphere. It follows (see Theorem 18.5 of [27]) that the closure of a cell in $G r(k, n)^{t n n}$ is the union of the cell itself with the lower dimensional cells.

For a given bipartite graph the image of the parametrization of the cell under the Plücker embedding is described by a map to the projective space. This map gives origin to a rational map from the toric variety to $\operatorname{Gr}(k, n)^{t n n}$. This map is well defined on the totally non negative part of the toric variety and the image of this map is the closure of the corresponding cell in the totally non negative Grassmannian. Since the totally non negative part of the toric variety is homeomorphic to the matching polytope the cell is parameterized by the polytope as well.

To conclude: the totally non negative part of the toric variety gets mapped to the closure of the cell and the totally positive part maps homeomorphically to the cell.

Two comments are in order.

First, by reducing the polytope on its faces one may find more subspaces than cells with a fixed dimensionality. Indeed it may happen that some of the sub-diagrams $\mathcal{H}$ give a different parameterization of the same cell. For example in this construction each 0dimensional cell corresponds to a single PM. On the other hand each 0-dimensional cell is

\footnotetext{
${ }^{10}$ Maybe it is worthwhile to remind the reader that the cells are subspaces where the non zero coordinates are strictly positive, and are isomorphically associated to the positive part of the toric variety associated to the sub-polytope. The closure of these cells provides some non trivial topological spaces that are the relevant ones for the scattering amplitudes.
} 
parameterized by a single Plücker coordinate. In general there are more PMs than Plücker coordinates: some of the PMs are associated to the same cell. They are the PMs that in the top cell differ by internal loops.

Second, as discussed in Corollary 7.4 of [41] in this reduction there are not cells that are not associated to at least one sub-diagram.

We can conclude that the projection of the matching polytope on its faces can be interpreted as a cell decomposition of $\operatorname{Gr}(k, n)^{t n n}$ in positive cells.

\section{$6 \quad$ Examples}

In this section we study examples of increasing complexity to elucidate the relation among the Grassmannian, the bipartite diagrams on the disks, and toric geometry. We start by revisiting in detail, by using the technology that we introduced in the last sections, the two examples already proposed in sections 2.1 and 2.2: namely the simple case of $G r(1,3)^{t n n}$, and then the bit more involved case of $\operatorname{Gr}(2,4)^{t n n}$ where the top cell is identified by a box bipartite graph. We study in great detail this last example, in particular we use the POs and PMs to assign the coordinates to the various cells and we study its cell decomposition. We then move to study some aspects of some subcells of new higher dimensional totally non negative Grassmannians.

\section{1 $\operatorname{Gr}(1,3)^{\operatorname{tnn}}$ and $\operatorname{Gr}(2,3)^{t n n}$}

The first two examples correspond to the graphs in (6.1)
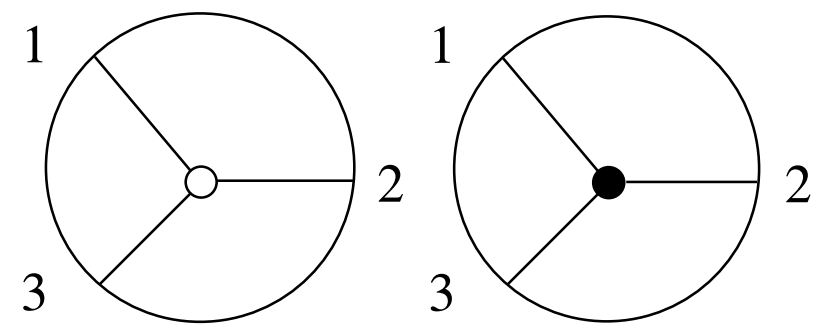

These graph are associated to the top cell of $G r(1,3)^{t n n}$ and $G r(2,3)^{t n n}$ respectively. Notice that since the Grassmannian is symmetric for $k \rightarrow n-k$, by studying one case one should be able to recover the other one. In both cases there are three PMs and POs as shown in $(6.2)$.
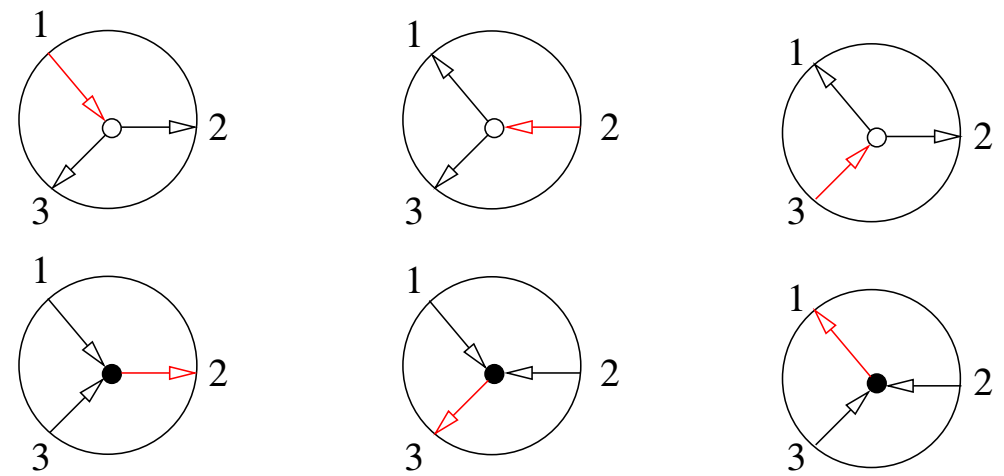
and there are no relations between the PMs in each case. The matching polytope is obtained from three vectors $v_{\sigma_{i}}=e_{i}$ and it coincides with the matroid polytope because there are not internal faces. The local Plücker coordinates are obtained as explained above. First one fixes a reference PO, the $i$-th, and then one gives the local description of the coordinates by setting to one the coordinate associated to the choice of the $i$-th PO. In the first case the $i$-th PM corresponds to the source set $I_{i}=\{i\}$. In every patch selected by the $i$-th PM the $j$-th Plücker coordinate (namely the ratio of the $j$-th Plücker coordinate over the $i$-th Plücker coordinate, that however it is equal to 1 in the $i$-th patch) is given by $\Delta_{j}=\frac{\pi_{j}}{\pi_{i}}$. A similar situation holds in the second case. We have

\begin{tabular}{|c|c|c|c|c|}
\hline Reference PO & Source & $\Delta_{12}$ & $\Delta_{13}$ & $\Delta_{23}$ \\
\hline first & $\{1,3\}$ & $\frac{\pi_{2}}{\pi_{1}}$ & 1 & $\frac{\pi_{3}}{\pi_{1}}$ \\
second & $\{1,2\}$ & 1 & $\frac{\pi_{1}}{\pi_{2}}$ & $\frac{\pi_{3}}{\pi_{2}}$ \\
third & $\{2,3\}$ & $\frac{\pi_{2}}{\pi_{3}}$ & $\frac{\pi_{1}}{\pi_{3}}$ & 1 \\
\hline
\end{tabular}

These are the three charts of $\mathbb{R P}_{\geq 0}^{2}$. The transition functions between the $i$-th and the $k$-th chart is given by the function $\frac{\pi_{i}}{\pi_{k}}$. In this way it is possible to reconstruct the full $\mathbb{R P}_{\geq 0}^{2}$ and reproduce its global description as presented in section 2.1.

\section{$6.2 \operatorname{Gr}(2,4)^{\operatorname{tnn}}$}

As we have previously explained in section 2.2 the top cell of $\operatorname{Gr}(2,4)^{\operatorname{tnn}}$ is identified by the box diagram in (2.8). This diagram describes the tree level scattering of four particles, two with positive helicities. In this section we study this diagram with the help of the toric technology we have introduced in the previous sections, with the aim to relate the toric description with the standard description of $G r(2,4)^{t n n}$ and its decomposition in positive cells as previously studied in $[26] .{ }^{11}$

First of all we have to identify the POs and the PMs $\sigma_{i}$. They are summarized together in $(6.3)$
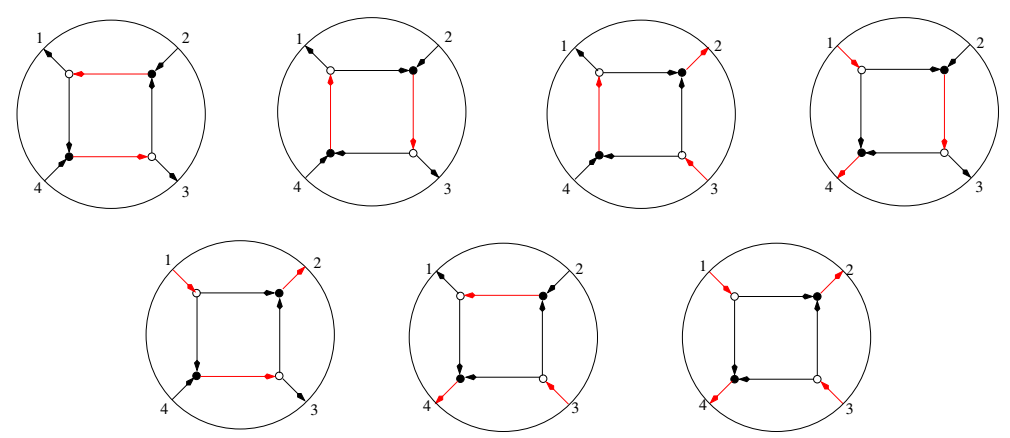

The relations (2.11) among the $\sigma_{i}$ generate the toric diagram defined by the vectors in equation (2.12). By following the procedure explained in the previous sections one can construct the relations (2.13) among the $\pi_{i}$ coordinates and hence the algebraic geometric description of the toric variety as intersection in $\mathbb{R P}_{\geq 0}^{6}$, that we re-propose here for

\footnotetext{
${ }^{11}$ See [] for an older study of the cell decomposition of $\operatorname{Gr}(2,4)$.
} 
completeness:

$$
\pi_{1} \pi_{7}=\pi_{5} \pi_{6}, \quad \pi_{2} \pi_{7}=\pi_{3} \pi_{4}
$$

and from here provide a global description of the associated $\operatorname{Gr}(2,4)^{\operatorname{tnn}}$ as we have already done in section 2.2 .

In this section we want instead take an alternative path and start from a local description, from patch to patch of the Grassmannian and the toric variety, and then reproduce the global description, by gluing together all the patches. This procedure should help us to gain a better insight in the correspondence between toric varieties and scattering amplitudes.

At the local level the Plücker coordinates are associated to the graph in the following way. As explained above every PM identifies a PO and consequently a source set. The graph in (2.8) represents the highest dimensional cell in the Grassmannian: namely the Grassmannian itself with strictly positive coordinates. Gluing together the different parametrizations of the top cell of the Grassmannian defined by all the possible set of sources, it is possible to reconstruct the closure of the cell and hence the totally non negative Grassmannian itself. Indeed by fixing a graph and a PO we give a description of the cell such that the coordinate associated to the source set is 1 . This is a local patch of the totally non negative Grassmannian and it was proven in [27] that in this patch the map between the underling toric variety and the Grassmannian can be uniquely extended, in a continuous way, to a map from the closure of the toric variety (namely allowing some of the coordinates of the perfect matching to go to zero) to the totally non negative Grassmannian. There are $\left(\begin{array}{l}n \\ k\end{array}\right)=6$ local maps that can be glued together to give a global map from $X_{\bar{P}}^{\geq 0}$ to $\operatorname{Gr}(k, n)^{t n n}$ that provides a global description of the closure of the cell itself.

We can indeed assign to every pair $\left(\mathcal{G}, P O_{\mathcal{G}}\right)$, representing a graph with a given orientation, the coordinates as explained in section 4.2. Furthermore it can be useful to translate this assignment to the face variables

The face variables are another useful set of coordinates that one can use to connect the bipartite diagrams with the Grassmannian. They are real non negative variables $f_{i}$ assigned to every face in the bipartite diagram that come with a counterclockwise orientation of the graph. Their product covers the whole disk, and it is normalized to be 1: namely $\prod_{k} f_{k}=1$, and there are indeed only $G-1$ free coordinates corresponding to the dimension of the closure of the cell associated to the on-shell diagram.

It is possible to connect the $\pi_{i}$ coordinates and the face variables $f_{i}$ as follows. First one fixes a reference PM, say $\sigma_{i}$, and the associated coordinate $\pi_{i}$, then one considers the ratios of the various $\mathrm{PM}$ coordinates $\pi_{j}$ divided by the reference PM coordinate $\pi_{i}$. The ratio $\pi_{j} / \pi_{i}$ is then the variable on the toric variety associated to a loop in the disk of the on-shell diagram (it can be a product of internal faces and loops ending on the boundary) and can be expressed as a product or ratio of face variables. Hence the face variables $f_{i}$ are a set of local coordinates for both the toric variety $X_{\bar{P}}^{>0}$ and the closure of the cell in the $\operatorname{Gr}(k, n)^{t n n}$, that are valid in the toric patch with $\pi_{i} \neq 0$. In addition they are quite special coordinates, because once we normalize $G-1$ of these coordinates $f_{j}$ dividing them by a reference face coordinate $f_{i}: \tilde{f}_{j}=f_{j} / f_{i}$, the invariant top form in $(2.2)$, used to link 
the Grassmannian to the scattering amplitudes, trivializes to a simple product $\prod_{j} d \tilde{f}_{j} / \tilde{f}_{j}$. For this reason the tilde face coordinates are also an interesting example of coordinates that are called canonical coordinates.

Let now see in detail, in the specific case of $G r(2,4)^{t n n}$, how the local map between the toric variety and the Grassmannian works, both in terms of the embedding coordinates $\pi_{j}$, and in terms of the local coordinates $f_{j}$. This map is explicitly shown in (6.5)

Let us comment on the coordinates assignation in (6.5). Every patch has been fixed by a PO. In this case there are seven possibilities, corresponding to the seven natural patches of the embedding space $\mathbb{R P}_{\geq 0}^{6}$ of the toric variety defined in (6.4). A PM, and hence a toric coordinate, corresponds to a PO. This coordinate is $\pi_{i}$ where the label $i$ represents the $i$-th toric patch defined by $\pi_{i} \neq 0$. At the same time one associates a set sources in the on-shell diagram to a PO. As previously explained the set of sources is in general smaller than the set of POs and hence in general more POs correspond to the same set source. A Plücker coordinate $p_{I}$ and a patch in the Plücker embedding space are associated to every source. In this case we have $\mathbb{R P}_{\geq 0}^{5}$, for which $p_{I} \neq 0$. The local coordinates $\Delta_{J}=p_{J} / p_{I}$. are defined in this $I$-th patch. The local maps shown in (6.5) are maps between the $i$-th patch in the toric variety and the $I$-th patch in the Grassmannian. The numerators and the denominators of the local coordinates $\Delta_{J}$ can be read from the procedure explained in section 4 as follows. One first looks at the structure of the incoming arrows in every PO listed in (6.5). If there is more than one PO with the same source set one has to consider the sum of the associated coordinates $\pi_{j}$. For every choice of $J$ one puts in the numerator the sum of the coordinates $\pi_{j}$ associated to the same source set. In the denominator the procedure works in the same way. This means that if the reference $i$-th PO is the only one associated to a choice $I$ of incoming indexes, than one divides by $\pi_{i}$. Otherwise if the $i$-th reference is not the only one associated to the set of incoming indexes parameterized by $I$, than one has to divide by the sum of $\pi_{i}$ with all the other coordinates $\pi_{k}$, with $k \neq i$ such that the related $k$-th POs share the same set of sources, namely the same structure of external indexes $I$. One can also parameterize the local coordinates $\Delta_{J}$ with the face variables. They are obtained by the ratios of the $\pi_{j}$ coordinates in the parameterization of $\Delta_{J}$ as previously explained

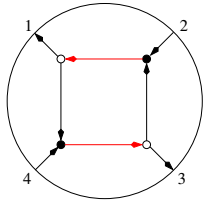

$$
\begin{gathered}
\Delta_{12}=\frac{\pi_{4}}{\pi_{1}+\pi_{2}} \quad \Delta_{13}=\frac{\pi_{7}}{\pi_{1}+\pi_{2}} \\
\Delta_{14}=\frac{\pi_{5}}{\pi_{1}+\pi_{2}} \Delta_{23}=\frac{\pi_{6}}{\pi_{1}+\pi_{2}} \\
\Delta_{24}=1 \quad \Delta_{34}=\frac{\pi_{3}}{\pi_{1}+\pi_{2}}
\end{gathered}
$$

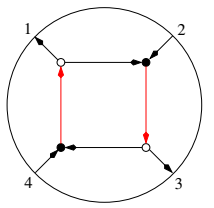

$$
\begin{gathered}
\Delta_{12}=\frac{\pi_{4}}{\pi_{1}+\pi_{2}} \Delta_{13}=\frac{\pi_{7}}{\pi_{1}+\pi_{2}} \\
\Delta_{14}=\frac{\pi_{5}}{\pi_{1}+\pi_{2}} \Delta_{23}=\frac{\pi_{6}}{\pi_{1}+\pi_{2}} \\
\Delta_{24}=1 \quad \Delta_{34}=\frac{\pi_{3}}{\pi_{1}+\pi_{2}}
\end{gathered}
$$

$$
\begin{aligned}
\Delta_{12}=\frac{f_{2} f_{3} f_{4}}{1+f_{1}^{-1}} \Delta_{13} & =\frac{f_{2} f_{4}}{1+f_{1}^{-1}} \\
\Delta_{14}=\frac{f_{2}}{1+f_{1}^{-1}} \Delta_{23} & =\frac{f_{4}}{1+f_{1}^{-1}} \\
\Delta_{24}=1 \quad \Delta_{34} & =\frac{f_{2} f_{4} f_{5}}{1+f_{1}^{-1}}
\end{aligned}
$$

$$
\begin{array}{cc}
\Delta_{12}=\frac{1}{f_{5}\left(1+f_{1}\right)} & \Delta_{13}=\frac{1}{f_{3} f_{5}\left(1+f_{1}\right)} \\
\Delta_{14}=\frac{1}{f_{3} f_{4} f_{5}\left(1+f_{1}\right)} & \Delta_{23}=\frac{1}{f_{2} f_{3} f_{5}\left(1+f_{1}\right)} \\
\Delta_{24}=1 & \Delta_{34}=\frac{1}{f_{3}\left(1+f_{1}\right)}
\end{array}
$$




$$
\begin{array}{cccc}
\Delta_{12}=\frac{\pi_{4}}{\pi_{3}} & \Delta_{13}=\frac{\pi_{7}}{\pi_{3}} & \Delta_{12}=\frac{f_{3}}{f_{5}} & \Delta_{13}=\frac{1}{f_{5}} \\
\Delta_{14}=\frac{\pi_{5}}{\pi_{3}} & \Delta_{23}=\frac{\pi_{6}}{\pi_{3}} & \Delta_{14}=\frac{1}{f_{4} f_{5}} & \Delta_{23}=\frac{1}{f_{2} f_{5}} \\
\Delta_{24}=\frac{\pi_{1}+\pi_{2}}{\pi_{3}} & \Delta_{34}=1 & \Delta_{24}=f_{3}+\frac{1}{f_{2} f_{4} f_{5}} & \Delta_{34}=1 \\
\Delta_{12}=1 & \Delta_{13}=\frac{\pi_{7}}{\pi_{4}} & \Delta_{12}=1 & \Delta_{13}=\frac{1}{f_{3}} \\
\Delta_{14}=\frac{\pi_{5}}{\pi_{4}} & \Delta_{23}=\frac{\pi_{6}}{\pi_{4}} & \Delta_{14}=\frac{1}{f_{3} f_{4}} & \Delta_{23}=\frac{1}{f_{2} f_{3}} \\
\Delta_{24}=\frac{\pi_{1}+\pi_{2}}{\pi_{4}} & \Delta_{34}=\frac{\pi_{3}}{\pi_{4}} & \Delta_{24}=f_{5}+\frac{1}{f_{2} f_{3} f_{4}} & \Delta_{34}=\frac{f_{5}}{f_{3}} \\
\Delta_{12}=\frac{\pi_{4}}{\pi_{5}} & \Delta_{13}=\frac{\pi_{7}}{\pi_{5}} & \Delta_{12}=f_{3} f_{4} & \Delta_{13}=f_{4} \\
\Delta_{14}=1 & \Delta_{23}=\frac{\pi_{6}}{\pi_{5}} & \Delta_{14}=1 & \Delta_{23}=\frac{f_{4}}{f_{2}} \\
\Delta_{24}=\frac{\pi_{1}+\pi_{2}}{\pi_{5}} & \Delta_{34}=\frac{\pi_{3}}{\pi_{5}} & \Delta_{24}=\frac{1}{f_{2}}+f_{3} f_{4} f_{5} & \Delta_{34}=f_{4} f_{5} \\
\Delta_{12}=\frac{\pi_{4}}{\pi_{6}} & \Delta_{13}=\frac{\pi_{7}}{\pi_{6}} & \Delta_{12}=f_{2} f_{3} & \Delta_{13}=f_{2} \\
\Delta_{14}=\frac{\pi_{5}}{\pi_{6}} & \Delta_{23}=1 & \Delta_{14}=\frac{f_{2}}{f_{4}} & \Delta_{23}=1 \\
\Delta_{24}=\frac{\pi_{1}+\pi_{2}}{\pi_{6}} \Delta_{34}=\frac{\pi_{3}}{\pi_{6}} & \Delta_{24}=\frac{1}{f_{4}}+f_{2} f_{3} f_{5} & \Delta_{34}=f_{2} f_{5} \\
\Delta_{12}=\frac{\pi_{4}}{\pi_{7}} & \Delta_{13}=1 & \Delta_{12}=f_{3} & \Delta_{13}=1 \\
\Delta_{14}=\frac{\pi_{5}}{\pi_{7}} & \Delta_{23}=\frac{\pi_{6}}{\pi_{7}} & \Delta_{14}=\frac{1}{f_{4}} & \Delta_{23}=\frac{1}{f_{2}} \\
\Delta_{24}=\frac{\pi_{1}+\pi_{2}}{\pi_{7}} & \Delta_{34}=\frac{\pi_{3}}{\pi_{7}} & \Delta_{24}=\frac{1}{f_{2} f_{4}}+f_{3} f_{5} & \Delta_{34}=f_{5}
\end{array}
$$
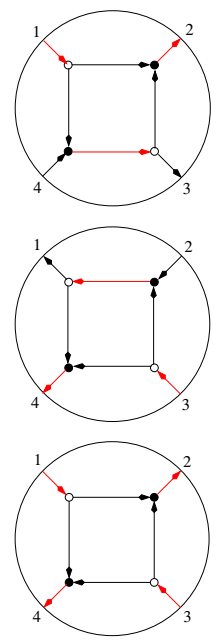

\subsubsection{Gluing the patches}

In the last subsection we discussed how to assign a local system of toric coordinates to a bipartite diagram that parameterizes a patch of the closure of a cell in the totally non negative Grassmannian. The system is local because every patch is selected by a reference PO and an associated set of non-zero coordinates, $\pi_{i}$ and $p_{I}$, both in the toric variety and in the cell in the Grassmannian. The $\Delta_{J}$ coordinates are ratios of the (sums of) $\pi_{j}$ coordinates assigned to the PMs. Every patch has one $\Delta_{J}$ coordinate, namely the one that fix the patch: $\Delta_{I}=p_{I} / p_{I}$, fixed to 1 . This is the coordinate related to the reference PO that fixes the patch itself.

The maps in (6.5) are in principle maps between the positive part of the toric variety (6.4), associated to the on-shell diagram, to the positive part of the Grassmannian $\operatorname{Gr}(2,4)$. Namely both the $\pi_{j}$ and the $\Delta_{J}$ are positive non zero variables.

To have global map between the non negative toric variety and the non negative Grassmannian, we should proceed in two steps: first of all extend the maps in (6.5) to their closure in every patch, and then glue all the patches together. As previously stated in [27] it was proven that these maps have a unique continuous extension. Hence following the discussion in section 4 one can glue the different patches by using the transition maps in formula (4.4) to obtain a global description of the parameterization of the closure of the cell associated to the bipartite diagram in the totally non negative Grassmannian, in this particular case of the full $\operatorname{Gr}(2,4)^{t n n}$. 
The transition map is obtained as explained in section (4.2) and considering that here $\Delta_{J}=p_{J} / p_{I}$ in the $I$-th patch. With the aim to clarify the discussion, let us introduce the notation $\Delta_{J}^{(I)}$ for the $p_{J}$ coordinate in the patch where $p_{I} \neq 0: \Delta_{J}^{(I)}=p_{J} / p_{I}$. Then the transition map between the $I$-th patch and the $K$-th patch is:

$$
\Delta_{J}^{(K)}=\frac{\sum_{\beta} \pi_{I}^{\beta}}{\sum_{\gamma} \pi_{K}^{\gamma}} \Delta_{J}^{(I)}
$$

Of course the $\pi_{j}$ coordinates in the previous transition function can be potentially defined on different toric patches of the toric variety, and when this happens, it is understood that every time it is considered the transition function $\pi_{i} / \pi_{k}$ between the $i$-th and the $k$-th toric patch.

One can assemble the transition functions $f_{I, K}$ in a matrix as follows:

$$
f_{I, K}=\left(\begin{array}{ccccccc}
\{2,4\} & \{3,4\} & \{1,2\} & \{1,4\} & \{2,3\} & \{1,3\} \\
\{2,4\} & 1 & \frac{\pi_{1}+\pi_{2}}{\pi_{3}} & \frac{\pi_{1}+\pi_{2}}{\pi_{4}} & \frac{\pi_{1}+\pi_{2}}{\pi_{5}} & \frac{\pi_{1}+\pi_{2}}{\pi_{6}} & \frac{\pi_{1}+\pi_{2}}{\pi_{7}} \\
\{3,4\} & \frac{\pi_{3}}{\pi_{1}+\pi_{2}} & 1 & \frac{\pi_{3}}{\pi_{4}} & \frac{\pi_{3}}{\pi_{5}} & \frac{\pi_{3}}{\pi_{6}} & \frac{\pi_{3}}{\pi_{7}} \\
\{1,2\} & \frac{\pi_{4}}{\pi_{1}+\pi_{2}} & \frac{\pi_{4}}{\pi_{3}} & 1 & \frac{\pi_{4}}{\pi_{5}} & \frac{\pi_{4}}{\pi_{6}} & \frac{\pi_{4}}{\pi_{7}} \\
\{1,4\} & \frac{\pi_{5}}{\pi_{1}+\pi_{2}} & \frac{\pi_{5}}{\pi_{3}} & \frac{\pi_{5}}{\pi_{4}} & 1 & \frac{\pi_{5}}{\pi_{6}} & \frac{\pi_{5}}{\pi_{7}} \\
\{2,3\} & \frac{\pi_{6}}{\pi_{1}+\pi_{2}} & \frac{\pi_{6}}{\pi_{3}} & \frac{\pi_{6}}{\pi_{4}} & \frac{\pi_{6}}{\pi_{5}} & 1 & \frac{\pi_{6}}{\pi_{7}} \\
\{1,3\} & \frac{\pi_{7}}{\pi_{1}+\pi_{2}} & \frac{\pi_{7}}{\pi_{3}} & \frac{\pi_{7}}{\pi_{4}} & \frac{\pi_{7}}{\pi_{5}} & \frac{\pi_{7}}{\pi_{6}} & 1
\end{array}\right)
$$

Each column and row of this matrix is associated to a set $I, K=\{.,$.$\} that specifies a$ patch and is associated to a reference PM or PO.

For example we can consider the transition function for the coordinate $\Delta_{34}$, between the second patch and the third patch in (6.5), defined respectively by the set of sources: (24) and (34): $\Delta_{34}^{(24)}$ and $\Delta_{34}^{(34)}$. In this case the transition map is obtained from the source set identified by the third and the second PO and we have:

$$
\Delta_{34}^{(34)}=\frac{\pi_{1}+\pi_{2}}{\pi_{3}} \Delta_{34}^{(24)}=1
$$

One can use these transition maps to glue the various patches.

These rules can be translated from the $\pi_{i}$ to the face variables as follows. First one defines the face variables in terms of the $\pi_{i}$ as

$$
f_{1}=\frac{\pi_{1}}{\pi_{2}}, \quad f_{2}=\frac{\pi_{5}}{\pi_{1}}=\frac{\pi_{7}}{\pi_{6}}, \quad f_{3}=\frac{\pi_{2}}{\pi_{3}}=\frac{\pi_{4}}{\pi_{7}}, \quad f_{4}=\frac{\pi_{7}}{\pi_{5}}=\frac{\pi_{6}}{\pi_{1}}, \quad f_{5}=\frac{\pi_{3}}{\pi_{7}}=\frac{\pi_{2}}{\pi_{4}}
$$

that automatically enforce the constraint $\prod f_{i}=1$. This constraint is useful in the gluing 
of the local patches. The transition map in (6.7) becomes

$$
f_{I, K}=\left(\begin{array}{ccccccc}
0 & \{2,4\} & \{3,4\} & \{1,2\} & \{1,4\} & \{2,3\} & \{1,3\} \\
\{2,4\} & 1 & f_{3}\left(f_{1}+1\right) & f_{5}\left(f_{1}+1\right) & \frac{f_{1}+1}{f_{1} f_{2}} & \frac{f_{1}+1}{f_{1} f_{4}} & f_{3} f_{5}\left(f_{1}+1\right) \\
\{3,4\} & \frac{1}{f_{3}\left(f_{1}+1\right)} & 1 & \frac{f_{5}}{f_{3}} & f_{4} f_{5} & f_{2} f_{5} & f_{5} \\
\{1,2\} & \frac{1}{f_{5}\left(f_{1}+1\right)} & \frac{f_{3}}{f_{5}} & 1 & f_{3} f_{4} & f_{2} f_{3} & f_{3} \\
\{1,4\} & \frac{f_{1} f_{2}}{f_{1}+1} & \frac{1}{f_{4} f_{5}} & \frac{1}{f_{3} f_{4}} & 1 & \frac{f_{2}}{f_{4}} & \frac{1}{f_{4}} \\
\{2,3\} & \frac{f_{1} f_{4}}{f_{1}+1} & \frac{1}{f_{2} f_{5}} & \frac{1}{f_{2} f_{3}} & \frac{f_{4}}{f_{2}} & 1 & \frac{1}{f_{2}} \\
\{1,3\} & \frac{1}{f_{3} f_{5}\left(f_{1}+1\right)} & \frac{1}{f_{5}} & \frac{1}{f_{3}} & f_{4} & f_{2} & 1
\end{array}\right)
$$

For example if we want to glue the patch identified by the fifth PM with the patch identified by the fourth one, we proceed as follows. First we write the $\Delta$ coordinates in each patch. The fifth PM selects the fourth row of the matrix, because it fixes the patch $I=\{1,4\}$. The third column is fixed by the fourth PM, associated to the sources $K=\{1,2\}$.

One starts by writing the Plücker coordinates $\Delta_{I}^{(1,4)}$ in terms of the faces

$$
\left\{\Delta_{12}^{(1,4)}, \Delta_{13}^{(1,4)}, \Delta_{14}^{(1,4)}, \Delta_{23}^{(1,4)}, \Delta_{24}^{(1,4)}, \Delta_{34}^{(1,4)}\right\}=\left\{f_{3} f_{4}, f_{4}, 1, \frac{f_{4}}{f_{2}}, \frac{1+f_{1}}{f_{1} f_{2}}, f_{4} f_{5}\right\}
$$

in the new patch fixed by $\pi_{4}$ they become

$$
\left\{\Delta_{12}^{(1,2)}, \Delta_{13}^{(1,2)}, \Delta_{14}^{(1,2)}, \Delta_{23}^{(1,2)}, \Delta_{24}^{(1,2)}, \Delta_{34}^{(1,2)}\right\}=\left\{1, \frac{1}{f_{3}}, \frac{1}{f_{3} f_{4}}, \frac{1}{f_{3} f_{2}}, f_{5}\left(1+f_{1}\right), \frac{f_{5}}{f_{3}}\right\}
$$

The transition map is the element $f_{4,3}$ of the matrix (6.10). It implies the relation

$$
\Delta_{J}^{(1,2)}=\frac{1}{f_{3} f_{4}} \Delta_{J}^{(1,4)}
$$

for every $J$. One can observe that this relation holds for every pair of coordinates in (6.11) and (6.12). The case $J=\{2,4\}$ needs an additional comment. In this case the equation (6.13) holds thanks to the constraint $\prod f_{i}=1$.

\subsubsection{Cell decomposition}

Up to now we discussed the parameterization of a cell and of its closure in the totally non negative Grassmannian $G r(k, n)^{t n n}$ in terms of the PM coordinates $\pi_{j}$ of a bipartite diagram on a disk. As we discussed in section 2 to connect these algebraic geometric varieties and the on-shell diagrams to the scattering amplitude processes it is important to study the decomposition of $G r(k, n)^{t n n}$ in subcells, and the related process in terms of toric geometry. The subcells are obtained by sending to zero some of the Plücker coordinates $p_{J}$, while forcing the remaining Plücker coordinates to be strictly positive, and preserving the positivity constraints. The union of the cells reconstructs the space itself. In appendix A.2.1 we will provide a more rigorous definition of the cell decomposition. Here we prefer to keep the discussion more intuitive because our main aim is to provide the cell decomposition in terms of bipartite diagrams and toric geometry. We refer the reader to the appendix and to $[27,41]$ for a more rigorous and detailed discussion. 
We already discussed the cell decomposition of $G r(2,4)^{t n n}$ in section 2.2. Here we will provide a detailed discussion of it in terms of toric geometry and the previously explained map between the PM coordinates and the Plücker coordinates.

As already explained in section 2 the reduction of the closure of the top cell, namely the totally non negative Grassmannian itself, into its subcells is related to the notion of removability of an edge. On the bipartite diagrams the notion of edge removability is defined in terms of the zig-zag paths. First one draws the zig-zag paths and then looks for the edges that correspond to single intersections of the paths. By removing one of those edges in the on-shell diagram, one identifies the new on-shell diagram associated to a subcell of the Grassmannian. At the level of the PMs it corresponds to erase the PMs $\sigma_{i}$ containing the removed edge. This procedure correspond to go on some facet of the matching polytope $P$, described by the vectors $v_{\sigma_{i}}$

The selected facet is associated to a toric variety in the usual way and this variety maps to the subcell of the Grassmannian associated to the same new on-shell diagram. For example in the on-shell diagram associated to the top cell of $\operatorname{Gr}(2,4)^{t n n}$ one can eliminate one of the four edges inside the box. There are four different graphs arising from this elimination as shown in (6.14).
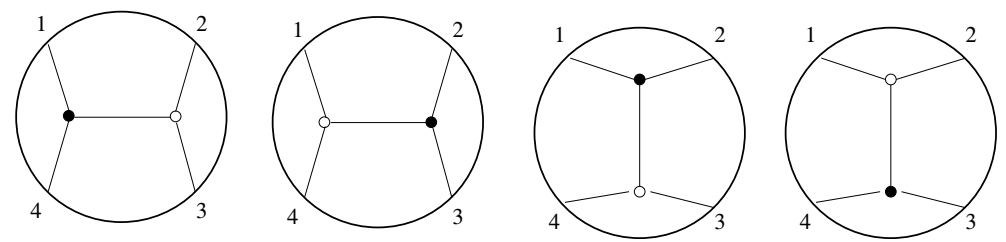

This process is usually formally interpreted in terms of matroids. Even if we will review the definition of the matroid in the appendix A, we prefer to give a short introduction to this object here. A matroid $\mathcal{M}$ of rank $k$ on the set $[n]$ is a non empty collection of $k$-element subset in $[n]$ such that for any $I$ and $J$ in $\mathcal{M}$ and $i \in I$ there is $j \in J$ such that $(I \backslash i) \bigcup j \in \mathcal{M}$. This property is called the exchange axiom. A matroid can be represented by the matroid polytope defined above.

The top cell identifies the single matroid with non-vanishing Plücker coordinates. In this case the matroid is called positroid. In principle there can be six matroids with five elements, such that in each of them all of the Plücker coordinates don't vanish but one. But only four of them satisfy the exchange axiom of the matroids. Indeed two of them should have had some negative or zero coordinates because of the Plücker relations. For example the matroid with $p_{13}=0$ would impose the constraint ${ }^{12}$

$$
p_{12} p_{34}+p_{14} p_{23}=0
$$

that cannot be satisfied for strictly positive values. The four choices that correspond to a matroid are the four graphs found by removing an internal edge each time.

Let us now fix one of the subcells, for example the one defined by $p_{23}=0$. In this case there are five surviving POs. In terms of the $\pi_{i}$ coordinates they are: $\pi_{2}, \pi_{3}, \pi_{4}, \pi_{5}, \pi_{7}$.

\footnotetext{
${ }^{12}$ Note that from now on we will work with the global coordinates $p_{I}$ without referring anymore to $\Delta_{I}$ coordinates and to the patches identified by some PO.
} 
There is one relation among them

$$
\pi_{2} \pi_{7}=\pi_{3} \pi_{4}
$$

and all the edges are removable.

Erasing one of them corresponds to eliminate some PMs. We can go on a subcell by eliminating one of the following sets of coordinates.

$$
\pi_{2} \& \pi_{3} \quad \text { or } \pi_{2} \& \pi_{4} \quad \text { or } \quad \pi_{3} \& \pi_{7} \quad \text { or } \quad \pi_{4} \& \pi_{7} \quad \text { or } \pi_{5}
$$

A similar situation holds on the other three subcells.

By eliminating $\pi_{2}$ and $\pi_{3}$ we set both $p_{24}$ and $p_{34}$ to zero. The non zero coordinates in this local parameterization of the subcell are $p_{12}, p_{13}$ and $p_{14}$, that satisfy the exchange axiom. By eliminating $\pi_{2}$ and $\pi_{4}$ the three non zero coordinates are $p_{13}, p_{14}$ and $p_{34}$, still a matroid. By eliminating $\pi_{3}$ and $\pi_{7}$ the three non zero coordinates are $p_{12}, p_{23}$ and $p_{24}$ and by eliminating $\pi_{4}$ and $\pi_{7}$ the three non zero coordinates are $p_{23}, p_{24}$ and $p_{34}$. The last possibility, $p_{14}=0$ is still a matroid. Differently from the first reduction from the top cell here we observe that some of the subcells have more than one extra vanishing Plücker coordinates to respect of the higher dimensional cell. In any case all of them satisfy the exchange axiom of the matroids.

\begin{tabular}{|c|c|c|c|}
\hline \multirow{5}{*}{$\begin{aligned} \pi_{1} & =0 \\
\pi_{5} & =0 \\
p_{14} & =0\end{aligned}$} & PM & Vanishing $p$ & Non-vanishing $p$ \\
\hline & $\pi_{2}=\pi_{3}=0$ & $p_{24}=p_{34}=0$ & $\left\{p_{12}, p_{13}, p_{23}\right\}$ \\
\hline & $\pi_{2}=\pi_{4}=0$ & $p_{24}=p_{12}=0$ & $\left\{p_{13}, p_{23}, p_{34}\right\}$ \\
\hline & $\pi_{3}=\pi_{7}=0$ & $p_{34}=p_{13}=0$ & $\left\{p_{12}, p_{23}, p_{24}\right\}$ \\
\hline & $\pi_{4}=\pi_{7}=0$ & $p_{12}=p_{13}=0$ & $\left\{p_{23}, p_{24}, p_{34}\right\}$ \\
\hline \multirow{6}{*}{$\begin{aligned} \pi_{1} & =0 \\
\pi_{6} & =0 \\
p_{23} & =0\end{aligned}$} & $\pi_{6}=0$ & $p_{23}=0$ & $\left\{p_{12}, p_{13}, p_{24}, p_{34}\right\}$ \\
\hline & PM & Vanishing $p$ & Non-vanishing $p$ \\
\hline & $\pi_{2}=\pi_{3}=0$ & $p_{24}=p_{34}=0$ & $\left\{p_{12}, p_{13}, p_{14}\right\}$ \\
\hline & $\pi_{2}=\pi_{4}=0$ & $p_{24}=p_{12}=0$ & $\left\{p_{13}, p_{14}, p_{34}\right\}$ \\
\hline & $\pi_{3}=\pi_{7}=0$ & $p_{34}=p_{13}=0$ & $\left\{p_{12}, p_{14}, p_{24}\right\}$ \\
\hline & $\pi_{4}=\pi_{7}=0$ & $p_{12}=p_{13}=0$ & $\left\{p_{14}, p_{24}, p_{34}\right\}$ \\
\hline \multirow{7}{*}{$\begin{aligned} \pi_{2} & =0 \\
\pi_{3} & =0 \\
p_{34} & =0\end{aligned}$} & $\pi_{5}=0$ & $p_{14}=0$ & $\left\{p_{12}, p_{13}, p_{24}, p_{34}\right\}$ \\
\hline & PM & Vanishing $p$ & Non-vanishing $p$ \\
\hline & $\pi_{1}=\pi_{5}=0$ & $p_{24}=p_{14}=0$ & $\left\{p_{12}, p_{13}, p_{23}\right\}$ \\
\hline & $\pi_{1}=\pi_{6}=0$ & $p_{24}=p_{23}=0$ & $\left\{p_{12}, p_{13}, p_{14}\right\}$ \\
\hline & $\pi_{5}=\pi_{7}=0$ & $p_{14}=p_{13}=0$ & $\left\{p_{12}, p_{23}, p_{24}\right\}$ \\
\hline & $\pi_{6}=\pi_{7}=0$ & $p_{23}=p_{13}=0$ & $\left\{p_{12}, p_{14}, p_{24}\right\}$ \\
\hline & $\pi_{4}=0$ & $p_{12}=0$ & $\left\{p_{13}, p_{14}, p_{23}, p_{24}\right\}$ \\
\hline
\end{tabular}

By considering all the possibilities we have 


\begin{tabular}{c|c|c|c|}
\cline { 2 - 4 } \multicolumn{1}{c|}{} & $\mathrm{PM}$ & Vanishing $p$ & Non-vanishing $p$ \\
\cline { 2 - 4 }$\pi_{2}=0$ & $\pi_{1}=\pi_{5}=0$ & $p_{24}=p_{14}=0$ & $\left\{p_{34}, p_{13}, p_{23}\right\}$ \\
$\pi_{4}=0$ & $\pi_{1}=\pi_{6}=0$ & $p_{24}=p_{23}=0$ & $\left\{p_{34}, p_{13}, p_{14}\right\}$ \\
$p_{12}=0$ & $\pi_{5}=\pi_{7}=0$ & $p_{14}=p_{13}=0$ & $\left\{p_{23}, p_{24}, p_{34}\right\}$ \\
& $\pi_{6}=\pi_{7}=0$ & $p_{23}=p_{13}=0$ & $\left\{p_{14}, p_{24}, p_{34}\right\}$ \\
$\pi_{4}=0$ & $p_{34}=0$ & $\left\{p_{13}, p_{14}, p_{23}, p_{24}\right\}$ \\
\hline
\end{tabular}

There are 20 matroids but not all of them are different. Two of them have four non zero coordinates, and both of them are obtained in two different ways. The other eight distinct matroids have three elements and each one appears twice. One can iterate this procedure to reach the lower dimensional cells until the zero dimensional cells, where just a single coordinate is non vanishing.

This section provided an explicit map from the cell decomposition of the Grassmannian to the partial facet decomposition of the matching polytope and hence to the partial decomposition of the non negative toric variety in some toric totally positive sub varieties.

\subsection{A subcell of $G r(3,6)^{\operatorname{tnn}}$}

In the previous sections, by making use of the toric geometry, we revisited the two examples: $\operatorname{Gr}(1,3)^{t n n}$ and $\operatorname{Gr}(2,4)^{t n n}$, that we have already discussed at the beginning of the paper. In this section we would like to study another more involved example, already discussed in [34]: a particular subcell of $\operatorname{Gr}(3,6)^{t n n}$. The aim of the section is to apply the algorithm that we developed to map the toric PM coordinates to the Plücker coordinates to a complex example and check its convenience.

The top cell of $\operatorname{Gr}(3,6)^{t n n}$ can be pictorially represented by the following diagram

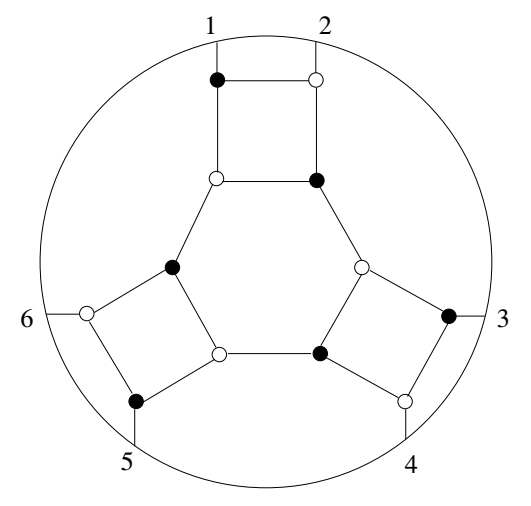

Here we are interested in a subcell, obtained by setting some edge variable to zero. After we do this some of the Plücker coordinates vanish and one ends up in a subcell. For example here we want to set to zero $p_{156}$ e $p_{345}$ and obtain the subcell. This is done by considering the two POs associated to these Plücker coordinates. These two POs are 
represented in (6.18)
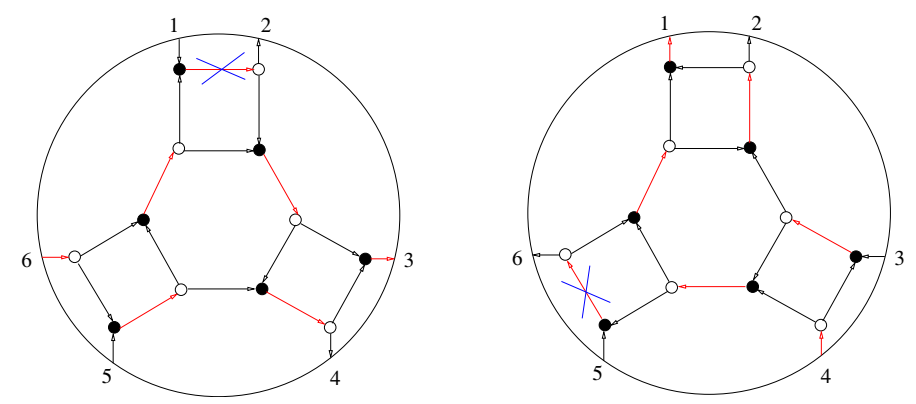

In (6.18) we also marked two edges in the top cell that have to be erased to obtain the subcell that we want to analyze. We eventually obtain the diagram in (6.19)

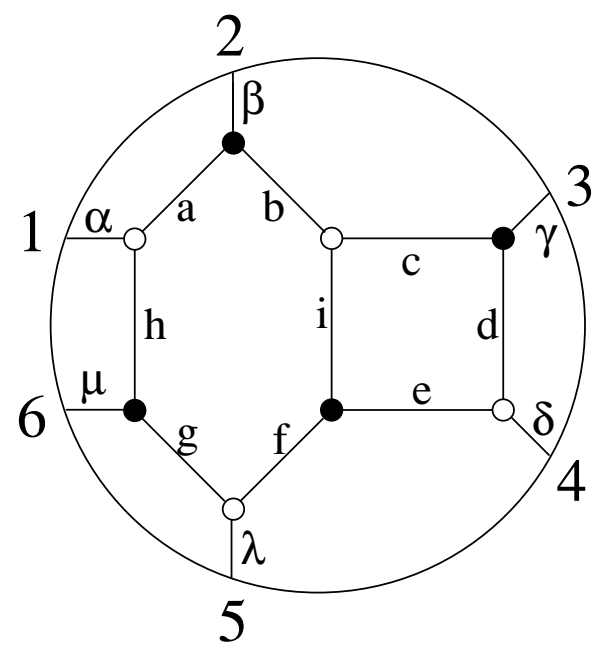

referred in [34] as the hexagon-box diagram. This on-shell diagram has 25 PMs (and POs). They can be represented graphically together as in (6.20).

The relations between the global coordinates on $G r(3,6)^{t n n}$ and the PM coordinates is

\begin{tabular}{|cc|cc|}
\hline Plücker & PM & Plücker & PM \\
$p_{123}$ & $\pi_{1}$ & $p_{124}$ & $\pi_{2}$ \\
$p_{125}$ & $\pi_{3}$ & $p_{126}$ & $\pi_{4}$ \\
$p_{134}$ & $\pi_{5}$ & $p_{135}$ & $\pi_{6}+\pi_{7}$ \\
$p_{136}$ & $\pi_{8}+\pi_{9}$ & $p_{145}$ & $\pi_{10}$ \\
$p_{146}$ & $\pi_{11}$ & $p_{156}$ & 0 \\
$p_{234}$ & $\pi_{12}$ & $p_{235}$ & $\pi_{13}+\pi_{14}$ \\
$p_{236}$ & $\pi_{15}+\pi_{16}+\pi_{17}$ & $p_{245}$ & $\pi_{18}$ \\
$p_{246}$ & $\pi_{19}+\pi_{20}$ & $p_{256}$ & $\pi_{21}$ \\
$p_{345}$ & 0 & $p_{346}$ & $\pi_{22}$ \\
$p_{356}$ & $\pi_{23}+\pi_{24}$ & $p_{456}$ & $\pi_{25}$ \\
\hline
\end{tabular}



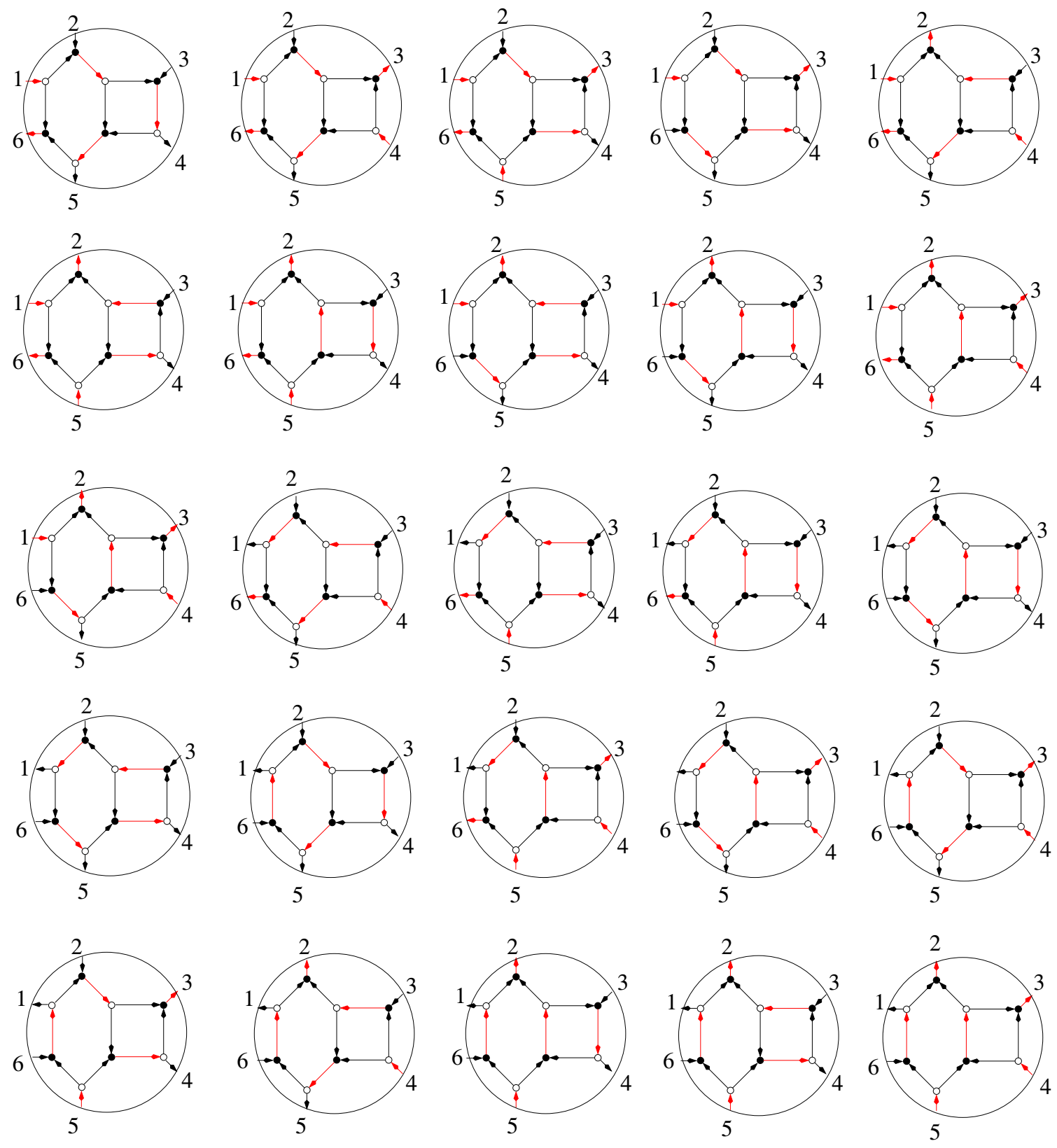

(6.20) 
From the defining equations of the toric variety associated to the on-shell diagram, one can also obtain the Plücker relation among the $p_{I}$ coordinates. This works as follows. First one has to construct the matching polytope. This is obtained by specifying the matrix of the PMs. In every row one fixes an edge in the diagram in (6.19) while in each column one fixes a PM, ordered as in (6.20). Each entry is 1 if the edge belongs to the PM, 0 otherwise

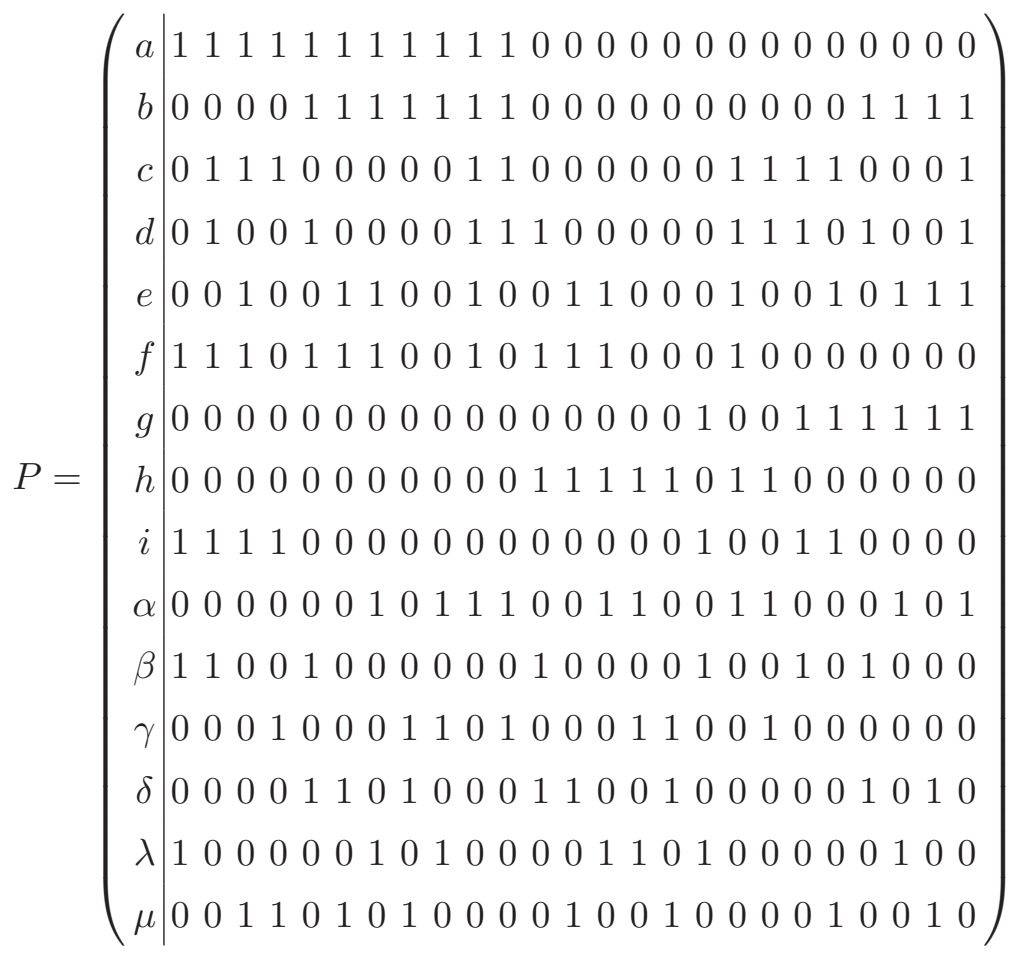

Not all the columns of this matrix are linearly independent. Indeed the kernel of this matrix is non empty and it identifies a set of linear relations among the PMs $\sigma_{i}$ and hence among the vectors $v_{\sigma_{i}}$ that define the matching polytope. A possible basis of relations is:

$$
\begin{array}{lll}
v_{\sigma_{1}}+v_{\sigma_{25}}=v_{\sigma_{7}}+v_{\sigma_{20}}, & v_{\sigma_{2}}+v_{\sigma_{24}}=v_{\sigma_{3}}+v_{\sigma_{22}}, & v_{\sigma_{1}}+v_{\sigma_{23}}=v_{\sigma_{7}}+v_{\sigma_{17}} \\
v_{\sigma_{1}}+v_{\sigma_{21}}=v_{\sigma_{17}}+v_{\sigma_{3}}, & v_{\sigma_{1}}+v_{\sigma_{20}}=v_{\sigma_{17}}+v_{\sigma_{2}}, & v_{\sigma_{5}}+v_{\sigma_{19}}=v_{\sigma_{11}}+v_{\sigma_{12}} \\
v_{\sigma_{2}}+v_{\sigma_{16}}=v_{\sigma_{4}}+v_{\sigma_{12}}, & v_{\sigma_{5}}+v_{\sigma_{15}}=v_{\sigma_{9}}+v_{\sigma_{12}}, & v_{\sigma_{5}}+v_{\sigma_{14}}=v_{\sigma_{7}}+v_{\sigma_{12}} \\
v_{\sigma_{2}}+v_{\sigma_{13}}=v_{\sigma_{3}}+v_{\sigma_{12}}, & v_{\sigma_{3}}+v_{\sigma_{11}}=v_{\sigma_{4}}+v_{\sigma_{10}}, & v_{\sigma_{1}}+v_{\sigma_{10}}=v_{\sigma_{2}}+v_{\sigma_{7}} \\
v_{\sigma_{3}}+v_{\sigma_{9}}=v_{\sigma_{4}}+v_{\sigma_{7}}, & v_{\sigma_{2}}+v_{\sigma_{8}}=v_{\sigma_{4}}+v_{\sigma_{5}}, & v_{\sigma_{2}}+v_{\sigma_{6}}=v_{\sigma_{3}}+v_{\sigma_{5}} \\
v_{\sigma_{1}}+v_{\sigma_{22}}=v_{\sigma_{17}}+v_{\sigma_{5}}, & v_{\sigma_{5}}+v_{\sigma_{18}}=v_{\sigma_{10}}+v_{\sigma_{12}}
\end{array}
$$

These relations translate in product relations among the real positive PM coordinates $\pi_{i}$ of the associated cell. The closure of these product relations (the toric ideal discussed in section 5) provide a set of polynomial equations for the toric variety associated to the on-shell diagram. From the relations between $\pi_{i}$ and $p_{I}$ one obtains the Plücker relations among the Plücker coordinates that parameterize the closure of this subcell of $G r(3,6)^{t n n}$. 


\subsection{Toric ideals and cell decomposition}

In this section we would like to discuss two further examples: $\operatorname{Gr}(3,5)^{t n n}$ and $G r(3,6)^{t n n}$. They are simple bipartite diagrams without internal loops. The aim of the section is to provide some explicit examples for the correspondence between the toric variety and the Grassmannian sub variety associated to the same on-shell diagram. The absence of internal loops allows a 1-1 identification between the Plücker coordinates and the PM. In addition it is possible to represent their toric diagram on a three dimensional lattice and their reduction in subcells can be explicitly observed on the polytope.

We start by defining the bipartite diagrams, and then we derive the the linear relations among the $v_{\sigma_{i}}$ variables defying the associated polytope. We then describe the multiplicative relations induced on the real positive PM coordinates $\pi_{i}$. In both the cases we show that the original linear relations among the PM are not enough to describe the projective toric variety as an intersection in the projective space. Namely new relations are generated in the process to take the closure of the map between the polytope and the $\pi_{i}$. We will then add the additional relations among the $\pi_{i}$ that allow to generate the toric ideal in both cases, and we explicitly show the toric interpretation of the associated scattering processes.

In the two examples we will present in the following, the matching polytope should be four dimensional: in both cases $G=4$. However the base of the cones lies on an hyperplane of codimension one: in both case $G-1=3$. This fact will allow us to explicitly draw the picture of the polytope as the three dimensional base of the associated cone. The fact that the vectors defining the matching polytope are coplanar is a generic phenomenon, as observed in [34], and proven in appendix B.

\subsubsection{Triangular basis}

The first example is a subcell of $G r(3,5)^{t n n}$ represented by the toric diagram and the PMs in (6.23).
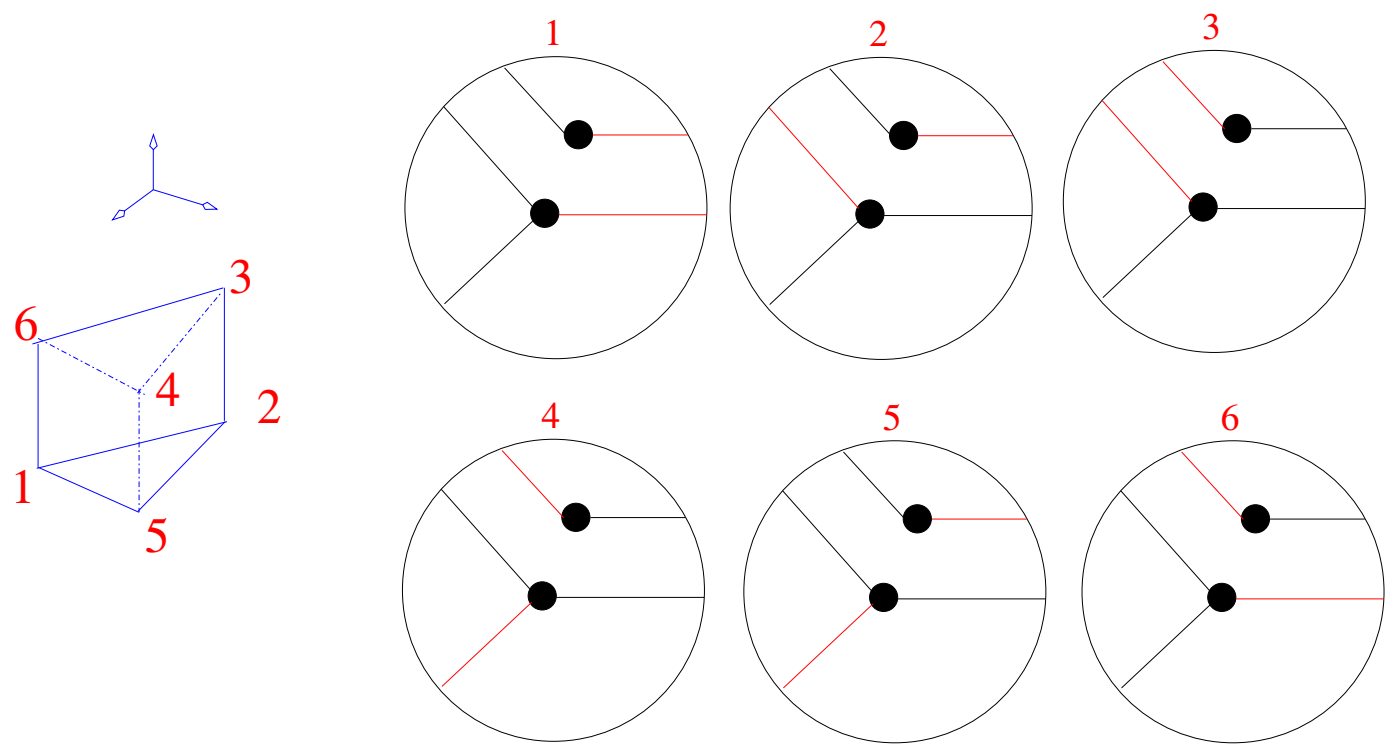

(6.23) 
The toric diagram can be defined by two relations among the perfect matchings. In terms of the vectors forming the toric diagram we have

$$
v_{\sigma_{1}}+v_{\sigma_{3}}=v_{\sigma_{2}}+v_{\sigma_{6}} \quad v_{\sigma_{2}}+v_{\sigma_{4}}=v_{\sigma_{3}}+v_{\sigma_{5}}
$$

Even if there are two relations among the vectors in the toric diagram the closure of the relations among the associated PM coordinates $\pi_{i}$ requires an additional relation. Indeed the toric ideal is generated by the three binomial relations:

$$
\pi_{1} \pi_{3}=\pi_{2} \pi_{6} \quad \pi_{2} \pi_{4}=\pi_{3} \pi_{5} \quad \pi_{5} \pi_{6}=\pi_{1} \pi_{4}
$$

The fact that there are three and not two relations in the ideal is crucial in understanding the decomposition in subcells.

The decomposition can be performed by erasing the edges. By following the procedure explained in [26] one observes that the subcells are in one to one correspondence with the five edges in (6.23). By erasing one edge and the PMs containing the edge itself one obtains the subcells. One can observe from (6.23) that by erasing one edge the only PMs that survive are always the ones associated to one of the five faces of the toric diagram: $F_{1456}, F_{4523}, F_{2316}, F_{643}$ and $F_{125}$, as expected.

One starts by sending to zero one $\pi_{i}$ in the three relations in (6.25). Some of these relations fail to be satisfied by positive numbers, and one is forced to send to zero also another $\pi_{i}$. One has to iterate the procedure until all the relations are satisfied or vanishing and the remaining PM coordinates $\pi_{i}$ define a codimension one polytope.

One can observe that this procedure gives the five faces in (6.23). In principle there could be internal faces in the polytope and this procedure may also end on some of them, that are not in principle associated to a subcell in the Grassmannian. In the next subsection we will study a case with internal faces.

\subsubsection{The cube}

The second example we want to study in this section is of a subcell of $\operatorname{Gr}(3,6)^{t n n}$. The bipartite on diagram is shown in (6.26)

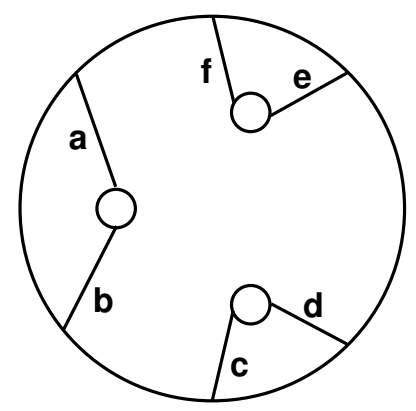


The PMs and the POs are shown in (6.27)
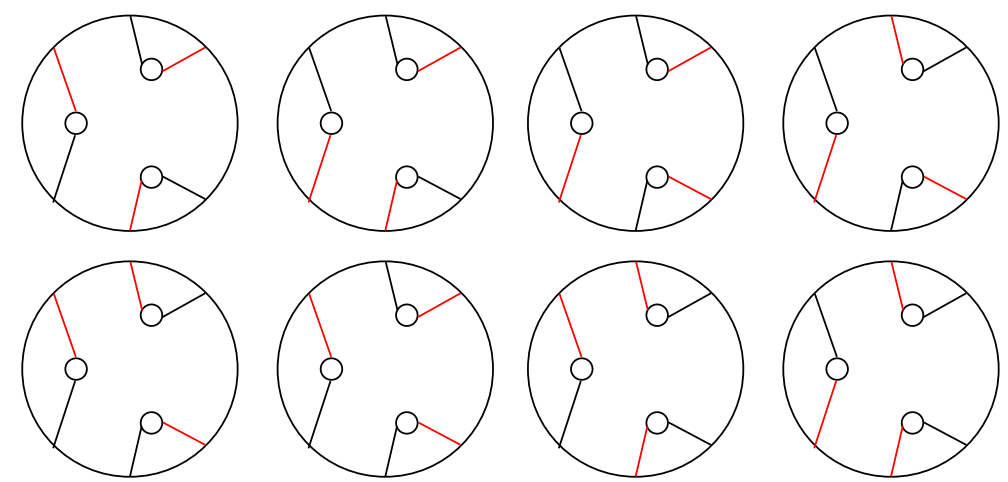

The matching polytope is

$$
P=\left(\begin{array}{l|llllllll}
a & 1 & 0 & 0 & 0 & 1 & 1 & 1 & 0 \\
b & 0 & 1 & 1 & 1 & 0 & 0 & 0 & 1 \\
c & 1 & 1 & 0 & 0 & 0 & 0 & 1 & 1 \\
d & 0 & 0 & 1 & 1 & 1 & 1 & 0 & 0 \\
e & 1 & 1 & 1 & 0 & 0 & 1 & 0 & 0 \\
f & 0 & 0 & 0 & 1 & 1 & 0 & 1 & 1
\end{array}\right)
$$

where in the rows are represented the edges and in the column the PMs. The base of the toric diagram corresponding to the matching polytope can be represented as
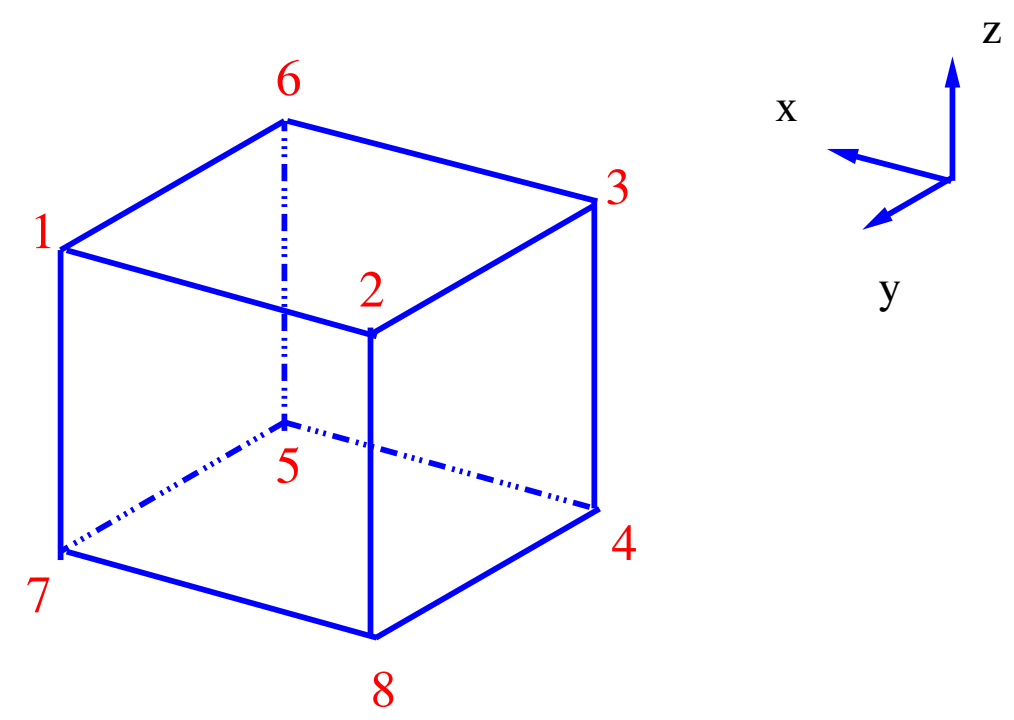

where the numbers are related to the PM ordered as in (6.27). The information contained in this diagram can be encoded in the following relations among the vectors forming the diagram

$v_{\sigma_{8}}+v_{\sigma_{3}}=v_{\sigma_{2}}+v_{\sigma_{4}}, \quad v_{\sigma_{7}}+v_{\sigma_{3}}=v_{\sigma_{1}}+v_{\sigma_{4}}, \quad v_{\sigma_{6}}+v_{\sigma_{2}}=v_{\sigma_{1}}+v_{\sigma_{3}}, \quad v_{\sigma_{5}}+v_{\sigma_{2}}=v_{\sigma_{1}}+v_{\sigma_{4}}$ 
As in the case studied above once we use the relations (6.30) to define the multiplicative relations among the real positive coordinates $\pi_{i}$, and we then take the closure of this map, allowing the $\pi_{i}$ to have zero values, we discover that the original relations are not enough and new relations are generated by the closure of the map. In this case indeed the toric ideal is generated by the following set of binomial quadratic relations that define the projective toric variety associated to the on-shell diagram:

$$
\begin{aligned}
& \pi_{4} \pi_{7}=\pi_{3} \pi_{8}, \quad \pi_{3} \pi_{7}=\pi_{6} \pi_{8}, \quad \pi_{2} \pi_{7}=\pi_{1} \pi_{8}, \quad \pi_{3} \pi_{5}=\pi_{4} \pi_{6}, \quad \pi_{2} \pi_{5}=\pi_{6} \pi_{8} \\
& \pi_{1} \pi_{5}=\pi_{6} \pi_{7}, \quad \pi_{2} \pi_{4}=\pi_{3} \pi_{8}, \quad \pi_{1} \pi_{4}=\pi_{6} \pi_{8}, \quad \pi_{1} \pi_{3}=\pi_{2} \pi_{6}
\end{aligned}
$$

As in the previous example the subcells are obtained either by erasing an edge in the on-shell diagram or by setting to zero the PM coordinates with the procedure explained above.

In this case there are internal faces in the matching polytope, but by sending to zero the PM coordinates $\pi_{i}$ in a way that preserve the relations (6.31) one ends up only on the external faces that describe the subcells.

\section{Dual diagrams and cluster transformations}

Until this point in this paper we have been discussing in detail the relation between an on-shell diagram and a cell in the totally non negative Grassmannian, the relation between an on-shell diagram and a projective toric variety, and the relation between the projective toric variety and the cell in the Grassmannian associated to the same on-shell diagram. However, as we have already commented at the very beginning of section 2, physically equivalent scattering processes are not associated to a single on-shell diagram, but to a class of on-shell diagrams: diagrams that can be mapped in other diagrams with mergers and square moves are physically equivalent, see section 2 . At this point it is maybe worthwhile to remind a series of correspondences proven in the mathematical literature (see for example [46]): decorated $(n, k)$ permutations are in one to one correspondence with cells in the non negative Grassmannian $G r(k, n)^{t n n}$; decorated $(k, n)$ permutation are in one to one correspondence with moves equivalent classes of on-shell diagrams.

Hence there should exist a well defined transformation among the geometric objects that we associate to different on-shell diagrams identified by the same permutation. Only the geometric structures that are invariant under such transformation are physically relevant to reconstruct the associated scattering process. This transformation is called cluster transformation. ${ }^{13}$

At the local level, namely considering strictly positive values for the coordinates of the cells in Grassmannian and of the toric variety associated to an on-shell diagram, an allowed move in the on-shell diagram corresponds to a particular change of coordinates in the cell in the Grassmannian and in the toric variety: the particular transformation is called cluster transformation. It works in the following way.

\footnotetext{
${ }^{13}$ from cluster algebra [51]. Here we will not discuss any mathematical detail of cluster algebra and refer the reader to the original paper for reference. Related discussions on the connection between the moves and the cluster transformations on bipartite diagrams on disks already appeared in [36, 38].
} 
A suitable set of coordinates for a cell in $G r(k, n)^{t n n}$ is provided by, for example, the face variables in the on-shell diagram. Face variables are indeed a good set of local coordinates both for the cell in Grassmannian and for the positive toric variety. To a single (reduced) on-shell diagram one can associate a cell in Grassmannian by using for example the map (4.1) between the edge or face variables for the on-shell diagram and the Plücker coordinates for the cell. Acting with mergers and square moves on the defined on-shell diagram one in general ends on another on-shell diagram. As we have just reminded a permutation is in one to one correspondence with a cell in Grassmannian and it is also in one to one correspondence with a class of equivalence of on-shell diagrams. This fact implies that the two cells in the Grassmannian provided for example by (4.1), associated to these two on-shell diagrams linked by a set of moves, should be isomorphic and hence the same cell. Indeed the on-shell diagram obtained by moves is in general different from the original one, for example the structure of the faces and the number of edges and PMs change. But with the use of (4.1) one can assign to this new diagram the same set of Plücker coordinates as the original one. This new diagram describes indeed the same point in the cell in $\operatorname{Gr}(k, n)^{t n n}$, but the Plücker coordinates are written in terms of different combinations of face variables. The transformation between the two sets of face variables are called cluster transformation.

We would like to link this local discussion with the global picture of the geometric structures associated to an on-shell diagram that we have been exploring in the rest of the paper. In this section indeed we would like to better understand what happen to this global picture under the allowed moves of the on-shell diagrams.

In particular to every on-shell diagram we have associated a matching polytope $P$ and a non negative real toric variety $X_{\bar{P}}^{\geq 0}$, and a map from this variety to the closure of the cell in $G r(k, n)^{t n n}$. In this section we would like to provide a preliminary analysis of the cluster transformation in this setup. A matching polytope $P$ is associated to every onshell diagram. Matching polytopes associated to on-shell diagrams related by mergers or square moves are not in general toric equivalent: namely there could not exist an $\operatorname{SL}(G, \mathbb{Z})$ transformation that maps the two polytopes one into the other, as extensively studied in the case of bipartite diagrams on the torus in [52]. However the projective toric varieties or their subsets that map to the cell in the Grassmannian should be related by some well defined transformation that locally reproduces the cluster transformation, that we have just discussed, among the face variables. In the following two examples we look for such correspondence.

Observe that even if the matching polytopes of two on-shell diagrams related by moves are not toric equivalent, their matroid polytope should be toric equivalent modulo $\operatorname{SL}\left(G-G_{\text {int }}, \mathbb{Z}\right)$. Indeed the matroid polytope could also be defined only in terms of matroid and Plücker coordinates that are blind to the moves of the on-shell diagrams (see for example [46]). Moreover the quiver field theory interpretation of the on-shell diagrams provided in $[34,37]$ supports this intuition. Hence locally on the toric projective variety the cluster transformations should behave somehow in a similar fashion of the ones discussed in [53]: namely a toric transformation on the matroid polytope and a non toric transformation on the fibers of the matching polytope, seen as the total space with base 
the matroid polytope. Exploring this connections would be very nice, but we leave it for future research. In this section empowered by this intuition we would limit ourselves to the exploration of a couple of examples.

Before starting our analysis we remind the reader how a cluster transformation acts on the face variables in a square move. The cluster transformation associated to a square move can be locally described on a piece of the bipartite on-shell diagram. One considers an internal face with four edges and represents the square move on the face variable $f$ as in $(7.1)^{14}$
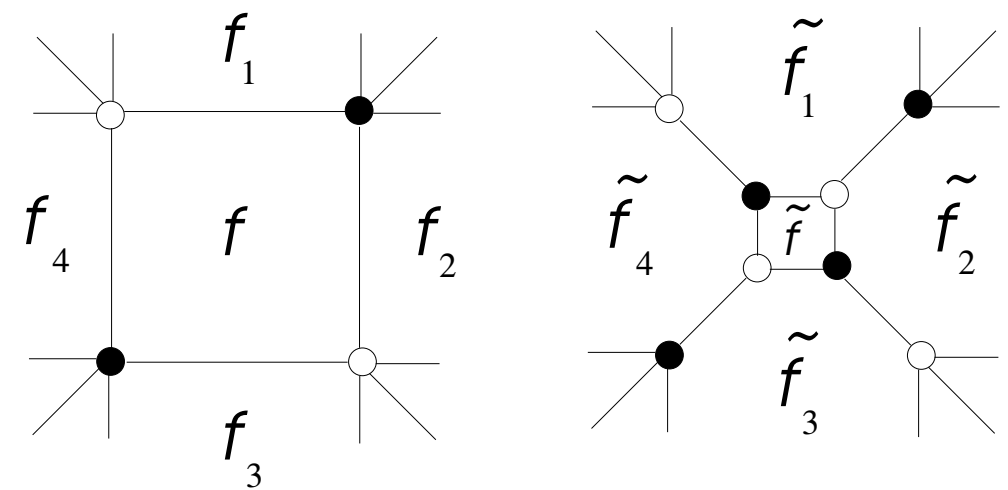

The corresponding cluster transformation is

$$
\tilde{f}=\frac{1}{f}, \quad \tilde{f}_{1}=\frac{f_{1}}{1+f^{-1}}, \quad \tilde{f}_{2}=f_{2}(1+f), \quad \tilde{f}_{3}=\frac{f_{3}}{1+f^{-1}}, \quad \tilde{f}_{4}=f_{4}(1+f)
$$

In the rest of the section we parameterize the cells and their closure in the totally non negative Grassmannian in terms of the PM coordinates $\pi_{i}$ as explained in the previous sections. This allows us to derive the cluster transformations (7.2) and deduce a set of "equivalence" transformations among the $\pi_{i}$ or subsets of them. Indeed we will observe that by imposing the equivalence of the Plücker coordinates for moves related on-shell diagrams in terms of the PM coordinates $\pi_{i}$ in the two phases we obtain a set of equations among (sums of) $\pi_{i}$ in the two phases. By translating these sets of equations in terms of the face variables in a local patch we will obtain the cluster transformations (7.2).

We will now apply these ideas to a pair of examples. We start by studying two diagrams associated to the top cell of $G r(2,4)^{t n n}$. Then we move to the subcell of $G r(3,6)^{t n n}$ referred as the hexagon- box diagram, and study its connection with its dual theory, obtained by applying a square move on the box.

\subsection{Cluster transformations in $\operatorname{Gr}(2,4)^{\operatorname{tnn}}$}

In the first example we by study the cluster transformation associated to a square move in the box diagram associated to the top cell of $G r(2,4)^{t n n}$, represented graphically in (2.8). By acting with a square move on the internal face of the box diagram we generate another box diagram with four external edges, but with the black and white vertexes exchanged,

\footnotetext{
${ }^{14}$ The transformation in (7.1) is apparently different from the one in (2.3). The reason is that here we are representing the most general effect of the square move on the face variables while in section 2 we just represented a simplified version, valid for some sub-cases, like $\operatorname{Gr}(2,4)^{t n n}$, for pedagocial reasons.
} 
see (7.3). We would like to study the cluster transformation on the face variables by analyzing the relation between the PM coordinates in the two phases and the associated Plücker coordinates. We call $\pi_{i}$ the PM coordinates associated to the original box on-shell diagram, and $\tilde{\pi}_{i}$ the PM coordinates of the on-shell box diagram obtained after the square move. We keep the convention that $\pi_{i}$ and $\tilde{\pi}_{i}$ are ordered as the associated PMs in (7.3) from 1 to 7 .
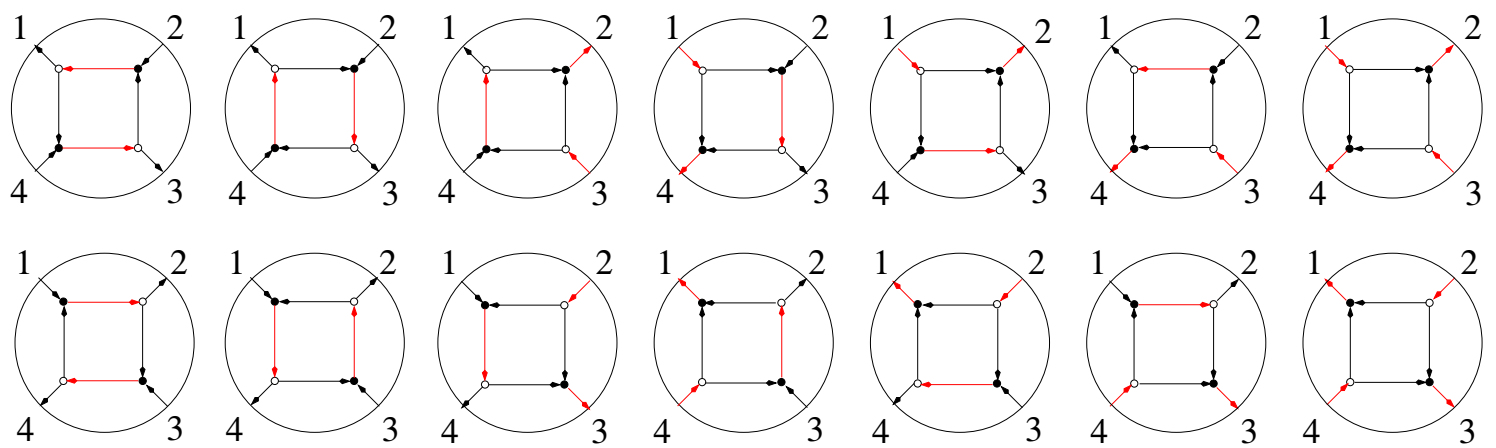

From the PMs one can read the POs and the corresponding source sets. Using equation (4.2) one then obtains the Plücker coordinates in terms of combinations of the PM coordinates for the two phases. If we impose that the two phases correspond to the same point in the top cell of $G r(2,4)^{t n n}$, as it should be thanks to the mathematical theorems stated in the previous section, we obtain a set of 6 equations relating the PMs in the two diagrams. Namely equating the (sums of) PMs coordinates associated to the same Plücker coordinate in the two move equivalent diagrams we obtain:

$$
\begin{aligned}
& p_{12}=\pi_{4}=\tilde{\pi}_{3} \\
& p_{13}=\pi_{7}=\tilde{\pi}_{1}+\tilde{\pi}_{2} \\
& p_{14}=\pi_{5}=\tilde{\pi}_{6} \\
& p_{23}=\pi_{6}=\tilde{\pi}_{5} \\
& p_{24}=\pi_{1}+\pi_{2}=\tilde{\pi}_{7} \\
& p_{34}=\pi_{3}=\tilde{\pi}_{4}
\end{aligned}
$$

This system of equations provides an equivalence relation for the toric varieties associated to the same scattering process. The next step consists of expressing the face variables in the two phases in terms of the PM coordinates. This is done by restricting to the totally positive part of the closure of the cell, i.e. all the coordinates are strictly positive and non zero. We have

$$
\begin{aligned}
& f=\frac{\pi_{1}}{\pi_{2}} \rightarrow \tilde{f}=\frac{\tilde{\pi}_{2}}{\tilde{\pi}_{1}} \\
& f_{1}=\frac{\pi_{5}}{\pi_{1}} \rightarrow \tilde{f}_{1}=\frac{\tilde{\pi}_{1}}{\tilde{\pi}_{5}} \\
& f_{2}=\frac{\pi_{2}}{\pi_{3}} \rightarrow \tilde{f}_{2}=\frac{\tilde{\pi}_{3}}{\tilde{\pi}_{2}} \\
& f_{3}=\frac{\pi_{6}}{\pi_{1}} \rightarrow \tilde{f}_{3}=\frac{\tilde{\pi}_{1}}{\pi_{6}} \\
& f_{4}=\frac{\pi_{2}}{\pi_{4}} \rightarrow \tilde{f}_{4}=\frac{\tilde{\pi}_{4}}{\tilde{\pi}_{2}}
\end{aligned}
$$


The relations (7.5) and (7.4) are still not enough to obtain the cluster transformations on the local face variables. We still have to consider the relations between the PMs coordinates in both the diagrams. They are:

$$
\pi_{7} \pi_{1}=\pi_{5} \pi_{6}, \quad \pi_{7} \pi_{2}=\pi_{3} \pi_{4}, \quad \tilde{\pi}_{7} \tilde{\pi}_{1}=\tilde{\pi}_{5} \tilde{\pi}_{6}, \quad \tilde{\pi}_{7} \tilde{\pi}_{2}=\tilde{\pi}_{3} \tilde{\pi}_{4}
$$

Using equation (7.5), (7.4) and (7.6) we can now reproduce the local cluster transformations among the face variables for the two phases (7.2). Explicitly we have:

$$
\begin{aligned}
& \tilde{f}=\frac{\tilde{\pi}_{2}}{\tilde{\pi}_{1}}=\frac{\tilde{\pi}_{3} \tilde{\pi}_{4}}{\tilde{\pi}_{5} \tilde{\pi}_{6}}=\frac{\pi_{3} \pi_{4}}{\pi_{5} \pi_{6}}=\frac{\pi_{2}}{\pi_{1}}=\quad \frac{1}{f} \\
& \tilde{f}_{1}=\frac{\tilde{\pi}_{1}}{\tilde{\pi}_{5}}=\frac{\tilde{\pi}_{6}}{\tilde{\pi}_{7}}=\frac{\pi_{5}}{\pi_{1}+\pi_{2}}=f_{1}\left(1+f^{-1}\right)^{-1} \\
& \tilde{f}_{2}=\frac{\tilde{\pi}_{3}}{\tilde{\pi}_{2}}=\frac{\tilde{\pi}_{7}}{\tilde{\pi}_{4}}=\frac{\pi_{1}+\pi_{2}}{\pi_{3}}=f_{2}(1+f) \\
& \tilde{f}_{3}=\frac{\tilde{\pi}_{6}}{\tilde{\pi}_{1}}=\frac{\tilde{\pi}_{5}}{\tilde{\pi}_{7}}=\frac{\pi_{6}}{\pi_{1}+\pi_{2}}=f_{3}\left(1+f^{-1}\right)^{-1} \\
& \tilde{f}_{4}=\frac{\tilde{\pi}_{4}}{\tilde{\pi}_{2}}=\frac{\tilde{\pi}_{7}}{\tilde{\pi}_{3}}=\frac{\pi_{1}+\pi_{2}}{\pi_{4}}=f_{4}(1+f)
\end{aligned}
$$

\subsection{A subcell of $\operatorname{Gr}(3,6)^{\operatorname{tnn}}$}

The previous example mapped two diagrams with substantially the same structure in terms of faces, edges and PMs.

Here we study a more complex example. Namely we apply the square move to the hexagon-box diagram, already discussed in section 6.3 and shown in (6.19), associated to a subcell of $\operatorname{Gr}(3,6)^{t n n}$. In this case the dual diagram, as already discussed in [34] is obtained by acting on the internal box with a square move. The dual diagram is a double box diagram with six external edges. This diagram has a different structure of faces, edges and PM with respect to the starting hexagon-box diagram and it is shown in (7.6)

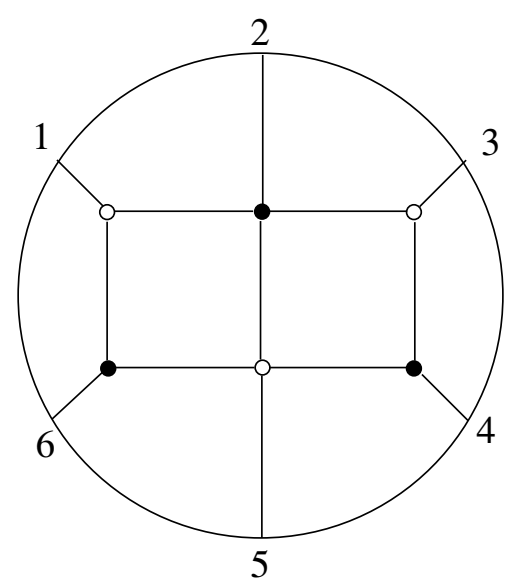

This diagram has 22 PMs and POs represented in (7.7), while the hexagon-box has 25 PMs 
and POs as shown in (6.20).

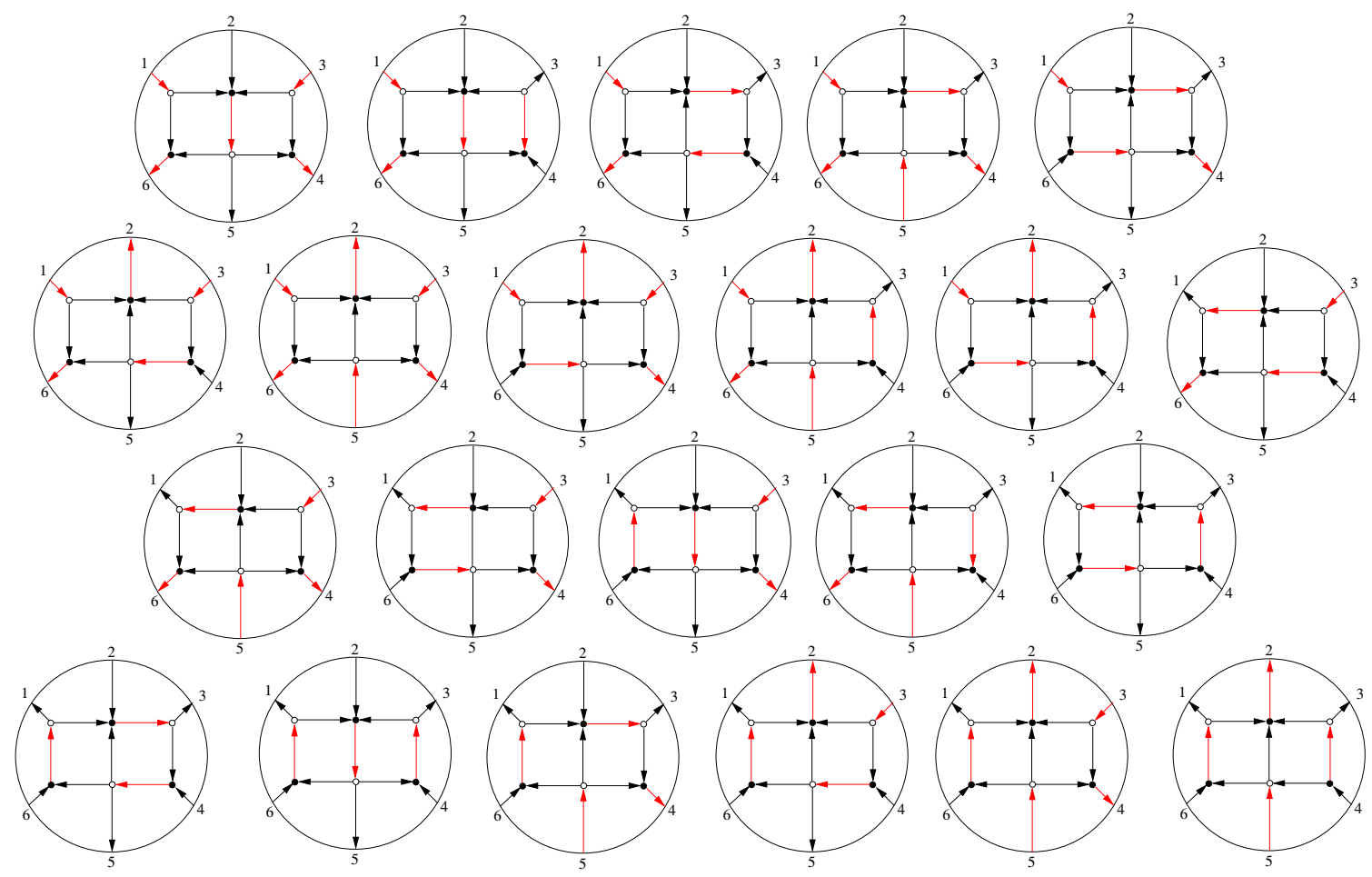

Each PM $\widetilde{\sigma}_{i}$ is associated to a vector $v_{\widetilde{\sigma}_{i}}$ in the matching polytope $\widetilde{P}$. These vectors satisfy the set of linear relations:

$$
\begin{array}{lll}
v_{\widetilde{\sigma}_{1}}+v_{\widetilde{\sigma}_{22}}=v_{\widetilde{\sigma}_{2}}+v_{\widetilde{\sigma}_{21}}, & v_{\widetilde{\sigma}_{3}}+v_{\widetilde{\sigma}_{21}}=v_{\widetilde{\sigma}_{4}}+v_{\widetilde{\sigma}_{20}}, & v_{\widetilde{\sigma}_{1}}+v_{\widetilde{\sigma}_{20}}=v_{\widetilde{\sigma}_{6}}+v_{\widetilde{\sigma}_{14}} \\
v_{\widetilde{\sigma}_{1}}+v_{\widetilde{\sigma}_{19}}=v_{\widetilde{\sigma}_{4}}+v_{\widetilde{\sigma}_{14}}, & v_{\widetilde{\sigma}_{1}}+v_{\widetilde{\sigma}_{18}}=v_{\widetilde{\sigma}_{2}}+v_{\widetilde{\sigma}_{14}}, & v_{\widetilde{\sigma}_{1}}+v_{\widetilde{\sigma}_{17}}=v_{\widetilde{\sigma}_{3}}+v_{\widetilde{\sigma}_{14}} \\
v_{\widetilde{\sigma}_{6}}+v_{\widetilde{\sigma}_{16}}=v_{\widetilde{\sigma}_{10}}+v_{\widetilde{\sigma}_{11}}, & v_{\widetilde{\sigma}_{6}}+v_{\widetilde{\sigma}_{15}}=v_{\widetilde{\sigma}_{9}}+v_{\widetilde{\sigma}_{11}}, & v_{\widetilde{\sigma}_{3}}+v_{\widetilde{\sigma}_{13}}=v_{\widetilde{\sigma}_{5}}+v_{\widetilde{\sigma}_{11}} \\
v_{\widetilde{\sigma}_{3}}+v_{\widetilde{\sigma}_{12}}=v_{\widetilde{\sigma}_{4}}+v_{\widetilde{\sigma}_{11}}, & v_{\widetilde{\sigma}_{1}}+v_{\widetilde{\sigma}_{10}}=v_{\widetilde{\sigma}_{2}}+v_{\widetilde{\sigma}_{8}}, & v_{\widetilde{\sigma}_{1}}+v_{\widetilde{\sigma}_{9}}=v_{\widetilde{\sigma}_{2}}+v_{\widetilde{\sigma}_{7}} \\
v_{\widetilde{\sigma}_{3}}+v_{\widetilde{\sigma}_{8}}=v_{\widetilde{\sigma}_{5}}+v_{\widetilde{\sigma}_{6}}, & v_{\widetilde{\sigma}_{3}}+v_{\widetilde{\sigma}_{7}}=v_{\widetilde{\sigma}_{4}}+v_{\widetilde{\sigma}_{6}}
\end{array}
$$

As usual these relations translate in product relations among the $\tilde{\pi}_{i}$ coordinates, and their closure generates the toric ideal.

Using equation (4.2) we obtain the relation between the global coordinates on the Grassmannian and the PMs coordinates in these square move equivalent on-shell diagrams:

\begin{tabular}{|cc|cc|cc|}
\hline Plücker & PM & Plücker & PM & Plücker & PM \\
$p_{123}$ & $\tilde{\pi}_{1}$ & $p_{124}$ & $\tilde{\pi}_{2}+\tilde{\pi}_{3}$ & $p_{125}$ & $\tilde{\pi}_{4}$ \\
$p_{126}$ & $\tilde{\pi}_{5} p_{134}$ & $\tilde{\pi}_{6}$ & $p_{135}$ & $\tilde{\pi}_{7}$ & \\
$p_{136}$ & $\tilde{\pi}_{8}$ & $p_{145}$ & $\tilde{\pi}_{9}$ & $p_{146}$ & $\tilde{\pi}_{10}$ \\
$p_{234}$ & $\tilde{\pi}_{11}$ & $p_{235}$ & $\tilde{\pi}_{12}$ & $p_{236}$ & $\tilde{\pi}_{13}+\tilde{\pi}_{14}$ \\
$p_{245}$ & $\tilde{\pi}_{15}$ & $p_{246}$ & $\tilde{\pi}_{16}+\tilde{\pi}_{17}+\tilde{\pi}_{18}$ & $p_{256}$ & $\tilde{\pi}_{19}$ \\
$p_{346}$ & $\tilde{\pi}_{20}$ & $p_{356}$ & $\tilde{\pi}_{21}$ & $p_{456}$ & $\tilde{\pi}_{22}$ \\
\hline
\end{tabular}


Now we proceed as before. We impose the equivalence between the (sums of) PM coordinates related to the same Plücker coordinate. These equations provide an equivalence relation between the two toric varieties associated to the same scattering process. We can then restrict the PM coordinates to have non zero positive values and express the face variables for the two phases in terms of ratios of PM coordinates. We can then study the relation between the face variables in the two phases as in the previous section. We order the faces as in (7.1) and we finally we find the expected cluster transformations among the face variables. Explicitly we have ${ }^{15}$

$$
\begin{aligned}
& \tilde{f}=\frac{\tilde{\pi}_{2}}{\tilde{\pi}_{3}}=\frac{\tilde{\pi}_{1} \tilde{\pi}_{9}}{\tilde{\pi}_{4} \tilde{\pi}_{6}}=\frac{\pi_{1} \pi_{10}}{\pi_{5} \pi_{3}}=\frac{\pi_{7}}{\pi_{6}}=\frac{1}{f} \\
& \tilde{f}_{1}=\frac{\tilde{\pi}_{4}}{\tilde{\pi}_{7}}=\frac{\pi_{3}}{\pi_{6}+\pi_{7}}=\frac{\pi_{3}}{\pi_{6}}\left(\frac{1}{1+\frac{\pi_{7}}{\pi_{6}}}\right)=f_{1}\left(1+f^{-1}\right)^{-1} \\
& \tilde{f}_{2}=\frac{\tilde{\pi}_{21}}{\tilde{\pi}_{22}}=\frac{\pi_{23}+\pi_{24}}{\pi_{25}}=\frac{\pi_{23}}{\pi_{25}}\left(1+\frac{\pi_{24}}{\pi_{23}}\right)=f_{2}(1+f) \\
& \tilde{f}_{3}=\frac{\tilde{\pi}_{6}}{\tilde{\pi}_{7}}=\frac{\pi_{5}}{\pi_{6}+\pi_{7}}=\frac{\pi_{5}}{\pi_{6}}\left(\frac{1}{1+\frac{\pi_{7}}{\pi_{6}}}\right)=f_{3}\left(1+f^{-1}\right)^{-1} \\
& f_{4}=\frac{\tilde{\pi}_{13}}{\tilde{\pi}_{14}}=\frac{\tilde{\pi}_{5} \tilde{\pi}_{6} \tilde{\pi}_{12}}{\tilde{\pi}_{1} \tilde{\pi}_{4} \tilde{\pi}_{20}}=\frac{\pi_{4} \pi_{5}\left(\pi_{13}+\pi_{14}\right)}{\pi_{1} \pi_{3} \pi_{22}}=\frac{\pi_{5}}{\pi_{17}} \frac{\pi_{13}+\pi_{14}}{\pi_{14}}=f_{4}(1+f)
\end{aligned}
$$

\section{Conclusions}

In this paper we started the investigation of the toric geometry underling the scattering amplitude processes in QFT. Our main aim was to provide an alternative description with the hope that it could bring some new insights in this fascinating field of research. In particular we explored the connection among the totally non negative Grassmannian, its cell decomposition, bipartite diagrams on disks and projective toric varieties. This was motivated by the connection between the on-shell diagrams representing the scattering amplitudes and the cell decomposition of the totally non negative Grassmannians. We believe that an interesting result of this paper is a map from the non negative real toric projective variety to the closure of the cells in $\operatorname{Gr}(k, n)^{t n n}$ associated to the same on-shell diagram, in terms of the global embedding coordinates $\pi_{i}$ and $p_{I}$. Thanks to this parameterization we studied the cell decomposition of $G r(k, n)^{t n n}$ in terms of toric geometry. Eventually we obtained the cluster transformations associated to the square moves in terms of toric geometry. We feel that there are various possible interesting developments to investigate. The most interesting line of research would be to investigate if the toric picture could help to reproduce the computation of already known scattering amplitudes or provide new results in the field.

Motivated by the connection between the top form of $G r(k, n)^{t n n}$ and the scattering amplitudes, and the relation between the toric varieties and the Grassmannians, one may think to some relation between the volumes of submanifolds of the toric variety and the Grassmannian integrand. For example, by interpreting the bipartite diagram on the

\footnotetext{
${ }^{15}$ Note that we obtained (7.10) thanks to the relations among the $\pi_{i}$ and $\tilde{\pi}_{i}$ variables in the toric ideal.
} 
disk as a supersymmetric field theory, as in [34-38], one can imagine a connection of the Grassmannian integrand with some toric localization procedures [54, 55].

We feel that another interesting topic regards the relation between the cluster transformations and the square moves. Indeed this is reminiscent of the gluing of the patches in the cluster Poisson varieties associated to the integrable systems living on the bipartite diagrams on the torus $[56,57]$. A first question is if a similar construction may be valid in the case of the disk. In the case of the torus it was observed that the master space, parameterized in terms of all the possible U(1) symmetries, mesonic and baryonic, anomalous and non anomalous, played a prominent role in the integrability of the dynamical system [53]. Here one may wonder about a similar role played by the matching polytope and in particular if the Poisson structure could play a role in the scattering processes [58].

It may be also interesting to study the connection between the removability of the edges and the toric geometry. Indeed here we still kept the usual definition of removability: an edge is removable if two zig-zag paths crossing on it do not cross on other edges. In principle it may be possible to understand the removability directly in terms of the toric variety, the constraints among the PMs and in terms of the relations between the PMs and the Plücker coordinates. Indeed after erasing a removable edge one ends up on a facet of the matching polytope, where some of the constraints among the PMs and some of the Plücker coordinates vanish. This suggests that the notion of removability maybe directly derived from the reduction of the matching polytope on its facets.

We could expect that an interesting role could be played by the specular duality [59] in the QFT interpretation of the on-shell diagrams. The specular duality, defined in the case of bipartite diagrams on the torus, relates the bipartite diagram with the so called mirror dual. After an untwisting procedure one construct the mirror diagram by assigning a face to each zig-zag path. This diagram is called mirror because it describes the IIA mirror geometry probed by a stack of D6 branes. The duality exchanges the baryonic and the mesonic symmetries, namely the external and the internal points of the toric diagram of the moduli space. It follows that it does not preserve the moduli space but it preserves the master space. In the case of reflexive polygons [60] this duality maps theories on the torus with theories on the torus. But in general, in non reflexive cases, it maps theories on the torus with theories on higher genus Riemann surfaces. It is natural to wonder if the specular duality plays any role in the case of the disk, by preserving the matching polytope. And, maybe more interesting, if it plays any role in the study of the non planar limit.

Another quite unexplored line of research in the quiver field theory interpretation of bipartite diagrams on disks is the relation between the zig-zag paths and the matroid polytope. Indeed in the case of bipartite diagrams on torus, the zig-zag paths are related to the mesonic moduli space of the associates quiver field theories living on D3 branes, through the $(p, q)$-web diagram. The mesonic moduli space is described by a toric diagram and the primitive normals of this diagram are related to the difference among consecutive external points, i.e. the zig-zag paths on the bipartite diagram. On the contrary one can build an inverse algorithm: from the toric diagram one can reconstruct the zig-zag paths and hence the bipartite diagram itself. In the case of the disk, one could wonder if a similar role is played by the zig-zag paths, but this interpretation is still lacking. 
We expect many exciting new lines of research both in the toric geometric description of scattering amplitudes and in the development of BFT.

\section{Acknowledgments}

We would like to thank Eduardo Conde Pena for his valuable participation in the early stage of this project and for many enlightening discussions. We would also like to kindly acknowledge F. Cachazo, E. C. Pena, and A. Zaffaroni, for their relevant comments and insights on the draft of the present paper. We are also grateful to R. Argurio, M. Bianchi, A. KashaniPoor for discussions and comments. A. A. is grateful to the Institut de Physique Théorique Philippe Meyer at the École Normale Supérieure for fundings. D. F. is a "Chargé de recherches" of the Fonds de la Recherche Scientifique-F.R.S.-FNRS (Belgium), and his research is supported by the F.R.S.-FNRS and partially by IISN - Belgium (conventions 4.4511.06 and 4.4514.08), by the "Communauté Française de Belgique" through the ARC program and by the ERC through the "SyDuGraM" Advanced Grant.

\section{A The totally non negative Grassmannian}

In the main part of the paper we studied the decomposition of the totally non negative Grassmannian in subspaces from the toric geometry perspective. The relation between toric diagrams and the totally non negative Grassmannians has been first noticed in [41].

Since we discussed many aspects of the Grassmannian that are not commonly used in physics we found it useful to review some basic definitions in this appendix.

We first review the basic definitions of the totally non negative Grassmannian and its cell decomposition. We then describe its connection to bipartite graphs as originally discussed in [27, 41].

\section{A.1 Grassmannians}

The Grassmannian $\operatorname{Gr}(k, n)$, with integers $n>k>0$, is the set of $k$-dimensional linear subspaces in $\mathbb{R}^{n}$ passing through the origin. Every point can be represented by a full rank $k \times n$ matrix $A$ modulo $G L(k)$ transformation. There exists a natural embedding of this space in a big enough projective space. First define $[n] \equiv\{1, \ldots, n\}$, and then a subset $I$ of $k$ elements of $[n]$. Then define $A_{I}$ the $k \times k$ submatrix of $A$ where the columns are labeled by $I$, there are $\left(\begin{array}{l}n \\ k\end{array}\right)$ of them. Then consider the determinants of this sub matrix. The Plücker coordinates are the determinants $p_{I}(A)=\operatorname{det} A_{I}$. These are projective coordinates, because the action of $G L(k)$ multiplies all the coordinates by a common factor. the map $A \rightarrow A_{I}$ induces the Plücker embedding of the real Grassmannian in $\mathbb{R P}\left(\begin{array}{l}n \\ k\end{array}\right)-1$. These coordinates are constrained by quadratic polynomial relations, called Plücker relations.

For example we consider $\operatorname{Gr}(2,4)$. In this case the matrix $\mathrm{A}$ is $4 \times 2$, and it can be represented in a local patch, thanks to the action of a $G L(2)$ transformation, as:

$$
A=\left(\begin{array}{cccc}
1 & a_{12} & 0 & -a_{14} \\
0 & a_{32} & 1 & a_{34}
\end{array}\right)
$$


The minors $\left(\Delta_{J}(A)=p_{J}(A) / p_{13}(A)\right.$, with $p_{13}(A)=1$ in this patch $)$ are

$$
\Delta_{12}=a_{32}, \quad \Delta_{13}=1, \quad \Delta_{14}=a_{34}, \quad \Delta_{23}=a_{12}, \quad \Delta_{24}=a_{12} a_{34}+a_{14} a_{32}, \quad \Delta_{34}=a_{14}
$$

and they are related by

$$
\Delta_{13} \Delta_{24}=\Delta_{12} \Delta_{34}+\Delta_{14} \Delta_{23}
$$

There is a subset of $\operatorname{Gr}(k, n)$ called the totally non negative Grassmannian $\operatorname{Gr}(k, n)^{t n n}$ represented by the matrix $A$ such that all the Plücker coordinates (the minors) are nonnegative. This space admits a nice quite simple cells decomposition. Indeed a simple way to decompose the totally non negative Grassmannian in cells is the so called positroid stratification. ${ }^{16}$

The positroid stratification can be defined as follows. First one defines the matroid. A matroid $\mathcal{M}$ of rank $k$ on the set $[n]$ is a non empty collection of $k$-element subset in $[n]$ such that for any $I$ and $J$ in $\mathcal{M}$ and $i \in I$ there is $j \in J$ such that $(I \backslash i) \bigcup j \in \mathcal{M}$. A positive cell $C_{\mathcal{M}}$ is the subset of elements in $\operatorname{Gr}(k, n)^{t n n}$ represented by all the matrices $A$ such that

$$
\left\{\begin{array}{l}
p_{I}>0 I \in \mathcal{M} \\
p_{I}=0 \text { otherwise }
\end{array}\right.
$$

If the cell is non empty the associated matroid is called positroid. $\operatorname{Gr}(k, n)^{\operatorname{tnn}}$ is a disjoint union of its cells. This cell decomposition of the totally non negative Grassmannian is crucial for the interpretation of planar bipartite graphs on disks in terms of scattering amplitudes.

\section{A.2 Bipartite diagrams and $G r(k, n)^{t n n}$}

A bipartite diagram on a disk can be connected with a subset in the totally non negative Grassmannian by making use of the boundary measurement.

Consider a PO in a graph with $n$ external vertices. This PO defines a set of $k$ sources, edges directed from the boundary to the internal, and $n-k$ sinks. For a given graph $k$ is independent from the choice of the PO. Assign a real positive variable $x_{e}$ to every edge in the diagram. If $i$ is in the source set and $j$ in the sink set one defines the boundary measurement as a series in $x_{e}$. Formally it is

$$
M_{i, j} \equiv \sum_{P(i \rightarrow j)}(-1)^{\operatorname{wind}(P)} x^{P}
$$

where $P$ are the directed paths from $i$ to $j$. The monomials $x^{P}$ are the ratios of the edge variables in every path: in the numerator there are edges directed from the white to the black vertices in the path and in the denominator edges from black to white. The winding index is the number of self intersections of a path modulo 2. If every edge variable is a positive real number the measurement is a positive function.

\footnotetext{
${ }^{16} \mathrm{It}$ is maybe important to underline that the positroid stratification is different from the usual Schubert cell decomposition of the Grassmannian as explained for example in [46].
} 
For a fixed PO, say $\mathcal{O}$, if one names the source set $I_{\mathcal{O}}$, one can construct a $k \times n$ matrix $A_{\mathcal{O}}$ with two properties. First the source set identifies a $k \times k$ minor corresponding to the identity. Second for any $i$ in the source set and $j$ in the sink set $\Delta_{\left(I_{\mathcal{O}} \backslash i\right) \cup j}\left(A_{\mathcal{O}}\right)=M_{i j}$. This construction defines the boundary measurement map MeasG that maps a perfectly oriented bipartite graph to a subset in the Grassmannian. Actually every PO underlies the same subset in $\operatorname{Gr}(k, n)$, i.e. the boundary measurement $M e a s_{\mathcal{G}}$ does not depend on the PO. The edge set is redundant, indeed there are gauge symmetries that one can use to reduce the boundary map $\operatorname{Meas}_{G}: R_{>0}^{E(\mathcal{G})} \rightarrow G r(k, n)^{t n n}$ to $\widetilde{M e a s_{\mathcal{G}}}: R_{>0}^{E(\mathcal{G})-V(\mathcal{G})} \rightarrow G r(k, n)^{t n n}$, or $\widetilde{\text { Meas }} \mathcal{G}: R_{>0}^{G(\mathcal{G})-1} \rightarrow G r(k, n)^{t n n}$, where $E, V$ and $G$ are respectively the number of edges, vertices and faces in the diagram.

\section{A.2.1 Cell decomposition and bipartite diagrams}

In the study of the scattering amplitudes it has been shown that the decomposition in positive cells of $\operatorname{Gr}(k, n)^{t n n}$ and the closure of these cells are the relevant geometric objects underling the scattering process. Here we briefly review this decomposition in terms of bipartite graphs.

One can associate to a bipartite graph $\mathcal{G}$ with a perfect orientation $\mathcal{O}$ a cell in $\operatorname{Gr}(k, n)^{t n n}$. This cell is parameterized by the Plücker coordinates as in [47], such that the Plücker coordinate associated to the source set of $\mathcal{O}$ is set to 1 . This is a local parameterization but one can move from one patch to another with a basis exchange: take an oriented paths in the $\mathrm{PO}$ and switch the orientation of every edge in the path.

The closure of the positive cell $C_{\mathcal{M}}$ associated to the graph $\mathcal{G}$ is obtained gluing together all these patches:

$$
\overline{C_{\mathcal{M}}}=\bigcup_{(\mathcal{G}, \mathcal{O})} \overline{\operatorname{Meas}_{(\mathcal{G}, \mathcal{O})}}\left(\mathbb{R}_{\geq 0}^{E(\mathcal{G})}\right)
$$

The closure of every boundary measurement $\overline{M e a s}_{(\mathcal{G}, \mathcal{O})}\left(\mathbb{R}_{\geq 0}^{E(\mathcal{G})}\right)$ is obtained from a perfectly oriented graph by allowing zero values for some edge(s). The union of all the measurement of those perfectly oriented subgraphs is the closure of the measurement of $(\mathcal{G}, \mathcal{O})$ and correspond to the closure of the cell $\bar{C}_{\mathcal{M}}$.

The edges that can be removed from $\mathcal{G}$ to construct a subgraph are named removable. An edge is removable if the two zig-zag paths that intersect on it do not intersect on any other edge in the graph. There is a 1-1 correspondence between the removable edges and the $(d-1)$-subcells covered by a $d$-cell. One can show that by further reducing the subgraph one arrives at the lower dimensional cells. This procedure is called the cell decomposition. The closure of the cell is the union of all its subcells.

\section{B Some further considerations}

In this appendix we group a couple of observations that come from our analysis.

First of all we argue that the vectors defining matching polytope are always coplanar and that as a consequences the matching polytope and the matroid polytope can be represented as polytopes in $G-1$ and $G-G_{\text {int }}-1$ dimensions respectively. This coplanarity 
condition has already been observed in [34] in all the examples that have been considered so far. We will prove this result in this section. This notion of coplanarity is well known in the case of bipartite diagrams on tori. Indeed in that case the toric diagram of the master space (the analogous of the matching polytope that we are considering here) is formed by vectors that lie on the same plane, as proven in [48]. This condition has been used to prove that the master space is a CY variety. Here we adapt the proof of the coplanarity of [48] to our case.

We start by observing that the PMs on the disk can be divided into two classes of edges

- Internal: edges connecting the vertices of the bipartite diagram.

- External: edges connecting a vertex in the bipartite diagram with the boundary.

Every PM covers all the vertices in the diagram. By assigning a weight 1 to the internal edges and $1 / 2$ to the external ones the weighted sum of the edges in every PM corresponds to the number of white (or black) vertices in the graph. This observation can be reformulated mathematically as follows. We first define the PM matrix $P$ as $P=\left(\begin{array}{c}P_{i} \\ P_{e}\end{array}\right)$ where $i$ runs over the internal edges and $e$ over the external ones. Then we have

$$
(1, \ldots, 1)_{E} \cdot\left(\begin{array}{cc}
I d_{i} & 0 \\
0 & \frac{1}{2} I d_{e}
\end{array}\right) \cdot P=\frac{V}{2}(1, \ldots, 1)_{c}
$$

From now on the proof goes along the lines of the one in [48]. By defining the matrix $Q$ as the kernel of $P: P \cdot Q=0$ we have

$$
(1, \ldots, 1)_{c} \cdot Q=0
$$

i.e. the rows of $Q^{t}$ are traceless, and all the vectors are coplanar.

The second thing we want to observe in this section is that the toric ideal of a toric variety associated to an on-shell diagram on a disk could be always generated by a set of quadratic binomials. This conclusion is obtained thanks to equation (4.2), that provides a linear map between the PM coordinates and the Plücker coordinates, and the fact that the Plücker embedding of the Grassmannian in the projective space is provided by a set of quadratic polynomials. The fact that the equations defining this kind of toric varieties are quadratic equations seems to be a peculiarity of this class of toric varieties associated to on-shell diagram on a disk. Indeed it is not true for general toric varieties, and for example it is not in general true for the toric varieties associated to a bipartite diagram on a torus. 


\section{List of notations}

\begin{tabular}{|c|c|}
\hline & Perfect matching variables with linear relations among them. \\
\hline$v_{\sigma_{i}}$ & Vectors associated to the PM. \\
\hline$\pi_{i}$ & Perfect matching coordinates with product relations among them. \\
\hline $\operatorname{Gr}(k, n)$ & Grassmannian manifold of $k$-dimensional linear subspaces of $\mathbb{R}^{n}$. \\
\hline$G r(k, n)^{t n n}$ & Totally non negative Grassmannian. \\
\hline $\mathcal{C}_{M}$ & (Totally positive) cell in the totally non negative Grassmannian \\
\hline $\mathcal{G}, \mathcal{H}$ & Bipartite graphs \\
\hline PM & Perfect matching \\
\hline $\mathrm{PO}$ & Perfect orientation \\
\hline & Number of PMs and POs. \\
\hline$G$ & Number of faces of a bipartite graph. \\
\hline$E$ & Number of external and internal edges in a bipartite graph. \\
\hline & Number of nodes in a bipartite graph. \\
\hline$F$ & Flow in a perfect orientation of a bipartite graph. \\
\hline$w(F)$ & Weight of a flow. \\
\hline$f_{i}$ & Face variables, such that $\prod_{i=1}^{G} f_{i}=1$. \\
\hline & Matroid. \\
\hline & Matching polytope, convex hull of the $c$ vectors $v_{\sigma}$. \\
\hline & Matroid polytope. \\
\hline$I=\left\{i_{1}, \ldots, i_{k}\right\}$ & Set of indices enumerating $k$ external lines of a bipartite diagram. \\
\hline 4 & Matrix representing a point in $G r(k, n)^{t n n}$. \\
\hline & Global Plücker coordinates. \\
\hline$\Delta_{I}$ & Local Plücker coordinates. \\
\hline$X_{P}$ & Projective Toric Variety. \\
\hline$X_{P}(\mathbb{R})$ & Real part of $X_{P}$. \\
\hline$X_{\bar{P}}^{\geq 0}$ & Totally non negative part of $X_{P}$. \\
\hline & Positive part of $X_{P}$ \\
\hline & Toric ideal of $X_{P}$. \\
\hline
\end{tabular}

Open Access. This article is distributed under the terms of the Creative Commons Attribution License which permits any use, distribution and reproduction in any medium, provided the original author(s) and source are credited.

\section{References}

[1] E. Witten, Perturbative gauge theory as a string theory in twistor space, Commun. Math. Phys. 252 (2004) 189 [hep-th/0312171] [INSPIRE]. 
[2] F. Cachazo, P. Svrček and E. Witten, $M H V$ vertices and tree amplitudes in gauge theory, JHEP 09 (2004) 006 [hep-th/0403047] [INSPIRE].

[3] Z. Bern, L.J. Dixon, D.C. Dunbar and D.A. Kosower, One loop $n$ point gauge theory amplitudes, unitarity and collinear limits, Nucl. Phys. B 425 (1994) 217 [hep-ph/9403226] [INSPIRE].

[4] Z. Bern, L.J. Dixon, D.C. Dunbar and D.A. Kosower, Fusing gauge theory tree amplitudes into loop amplitudes, Nucl. Phys. B 435 (1995) 59 [hep-ph/9409265] [INSPIRE].

[5] R. Britto, F. Cachazo and B. Feng, Generalized unitarity and one-loop amplitudes in $N=4$ super-Yang-Mills, Nucl. Phys. B 725 (2005) 275 [hep-th/0412103] [INSPIRE].

[6] R. Britto, F. Cachazo and B. Feng, New recursion relations for tree amplitudes of gluons, Nucl. Phys. B 715 (2005) 499 [hep-th/0412308] [INSPIRE].

[7] R. Britto, F. Cachazo, B. Feng and E. Witten, Direct proof of tree-level recursion relation in Yang-Mills theory, Phys. Rev. Lett. 94 (2005) 181602 [hep-th/0501052] [INSPIRE].

[8] Z. Bern, L.J. Dixon and D.A. Kosower, On-shell recurrence relations for one-loop QCD amplitudes, Phys. Rev. D 71 (2005) 105013 [hep-th/0501240] [INSPIRE].

[9] E.I. Buchbinder and F. Cachazo, Two-loop amplitudes of gluons and octa-cuts in $N=4$ super Yang-Mills, JHEP 11 (2005) 036 [hep-th/0506126] [INSPIRE].

[10] P. Benincasa and F. Cachazo, Consistency conditions on the S-matrix of massless particles, arXiv:0705.4305 [INSPIRE].

[11] M. Bianchi, H. Elvang and D.Z. Freedman, Generating tree amplitudes in $N=4 S Y M$ and $N=8 S G, J H E P 09$ (2008) 063 [arXiv:0805.0757] [inSPIRE].

[12] J. Drummond, J. Henn, G. Korchemsky and E. Sokatchev, Dual superconformal symmetry of scattering amplitudes in $N=4$ super-Yang-Mills theory, Nucl. Phys. B 828 (2010) 317 [arXiv:0807.1095] [INSPIRE].

[13] N. Arkani-Hamed, F. Cachazo and J. Kaplan, What is the simplest quantum field theory?, JHEP 09 (2010) 016 [arXiv:0808.1446] [INSPIRE].

[14] J. Drummond and J. Henn, All tree-level amplitudes in $N=4$ SYM, JHEP 04 (2009) 018 [arXiv:0808.2475] [INSPIRE].

[15] J.M. Drummond, J.M. Henn and J. Plefka, Yangian symmetry of scattering amplitudes in $N=4$ super Yang-Mills theory, JHEP 05 (2009) 046 [arXiv:0902.2987] [INSPIRE].

[16] R.H. Boels, On BCFW shifts of integrands and integrals, JHEP 11 (2010) 113 [arXiv: 1008.3101] [INSPIRE].

[17] L. Mason and D. Skinner, The complete planar S-matrix of $N=4$ SYM as a Wilson loop in twistor space, JHEP 12 (2010) 018 [arXiv: 1009.2225] [INSPIRE].

[18] S. Caron-Huot, Notes on the scattering amplitude/Wilson loop duality, JHEP 07 (2011) 058 [arXiv: 1010.1167] [INSPIRE].

[19] J. Drummond, J. Henn, V. Smirnov and E. Sokatchev, Magic identities for conformal four-point integrals, JHEP 01 (2007) 064 [hep-th/0607160] [INSPIRE].

[20] L.F. Alday and J.M. Maldacena, Gluon scattering amplitudes at strong coupling, JHEP 06 (2007) 064 [arXiv: 0705.0303] [INSPIRE]. 
[21] N. Arkani-Hamed, F. Cachazo, C. Cheung and J. Kaplan, A duality for the S matrix, JHEP 03 (2010) 020 [arXiv:0907.5418] [INSPIRE].

[22] N. Arkani-Hamed, J.L. Bourjaily, F. Cachazo, S. Caron-Huot and J. Trnka, The all-loop integrand for scattering amplitudes in planar $N=4$ SYM, JHEP 01 (2011) 041 [arXiv: 1008.2958] [INSPIRE].

[23] J. Drummond and L. Ferro, The Yangian origin of the Grassmannian integral, JHEP 12 (2010) 010 [arXiv: 1002.4622] [INSPIRE].

[24] J. Drummond and L. Ferro, Yangians, Grassmannians and T-duality, JHEP 07 (2010) 027 [arXiv: 1001.3348] [INSPIRE].

[25] N. Beisert, J. Henn, T. McLoughlin and J. Plefka, One-loop superconformal and Yangian symmetries of scattering amplitudes in $N=4$ super Yang-Mills, JHEP 04 (2010) 085 [arXiv: 1002 .1733] [INSPIRE].

[26] N. Arkani-Hamed et al., Scattering amplitudes and the positive Grassmannian, arXiv: 1212.5605 [INSPIRE].

[27] A. Postnikov, Total positivity, Grassmannians and networks, math/0609764.

[28] A. Hanany and K.D. Kennaway, Dimer models and toric diagrams, hep-th/0503149 [INSPIRE].

[29] S. Franco, A. Hanany, K.D. Kennaway, D. Vegh and B. Wecht, Brane dimers and quiver gauge theories, JHEP 01 (2006) 096 [hep-th/0504110] [INSPIRE].

[30] A. Hanany and D. Vegh, Quivers, tilings, branes and rhombi, JHEP 10 (2007) 029 [hep-th/0511063] [INSPIRE].

[31] B. Feng, Y.-H. He, K.D. Kennaway and C. Vafa, Dimer models from mirror symmetry and quivering amoebae, Adv. Theor. Math. Phys. 12 (2008) 489 [hep-th/0511287] [INSPIRE].

[32] S. Franco et al., Gauge theories from toric geometry and brane tilings, JHEP 01 (2006) 128 [hep-th/0505211] [INSPIRE].

[33] A. Butti, D. Forcella and A. Zaffaroni, The dual superconformal theory for $L^{p, q, r}$ manifolds, JHEP 09 (2005) 018 [hep-th/0505220] [INSPIRE].

[34] S. Franco, Bipartite field theories: from D-brane probes to scattering amplitudes, JHEP 11 (2012) 141 [arXiv: 1207.0807] [INSPIRE].

[35] D. Xie and M. Yamazaki, Network and Seiberg duality, JHEP 09 (2012) 036 [arXiv: 1207.0811] [INSPIRE].

[36] J.J. Heckman, C. Vafa, D. Xie and M. Yamazaki, String theory origin of bipartite SCFTs, JHEP 05 (2013) 148 [arXiv:1211.4587] [INSPIRE].

[37] S. Franco, D. Galloni and R.-K. Seong, New directions in bipartite field theories, JHEP 06 (2013) 032 [arXiv:1211.5139] [INSPIRE].

[38] S. Franco, Cluster transformations from bipartite field theories, arXiv:1301.0316 [INSPIRE].

[39] S. Cremonesi, A. Hanany and R.-K. Seong, Double handled brane tilings, arXiv:1305.3607 [INSPIRE].

[40] N. Arkani-Hamed, Scattering amplitudes and the positive Grassmannian, talk at Strings, Germany (2012).

[41] A. Postnikov, D. Speyer and L. Williams, Matching polytopes, toric geometry and the non-negative part of the Grassmannian, arXiv:0706.2501. 
[42] I. Gelfand and R.D. MacPherson, Geometry in Grassmannians and a generalization of the dilogarithm, Adv. Math. 44 (1982) 279.

[43] N. Seiberg and E. Witten, Electric-magnetic duality, monopole condensation and confinement in $N=2$ supersymmetric Yang-Mills theory, Nucl. Phys. B 426 (1994) 19 [Erratum ibid. B 430 (1994) 485] [hep-th/9407087] [INSPIRE].

[44] N. Seiberg, Electric-magnetic duality in supersymmetric non-Abelian gauge theories, Nucl. Phys. B 435 (1995) 129 [hep-th/9411149] [INSPIRE].

[45] J.M. Maldacena, The large- $N$ limit of superconformal field theories and supergravity, Adv. Theor. Math. Phys. 2 (1998) 231 [Int. J. Theor. Phys. 38 (1999) 1113] [hep-th/9711200] [INSPIRE].

[46] A. Postnikov, Topics in combinatorics positive Grassmannian, lectures at MIT Spring 2012, U.S.A. (2012).

[47] K. Talaska, A formula for Plücker coordinates associated with a planar network, arXiv:0801.4822.

[48] D. Forcella, A. Hanany, Y.-H. He and A. Zaffaroni, The master space of $N=1$ gauge theories, JHEP 08 (2008) 012 [arXiv:0801.1585] [INSPIRE].

[49] D. Cox, Topics in algebraic geometry and geometric modeling, Contemp. Math. 334 (2003) 203, Amer. Math. Soc., Providence U.S.A. (2003).

[50] F. Sottile, Topics in algebraic geometry and geometric modeling, Contemp. Math. 334 (2003) 225, Amer. Math. Soc., Providence U.S.A. (2003).

[51] S. Fomin and A. Zelevinsky, Cluster algebras, I. Foundations, J. Am. Math. Soc. 15 (2002) 497.

[52] D. Forcella, A. Hanany and A. Zaffaroni, Master space, Hilbert series and Seiberg duality, JHEP 07 (2009) 018 [arXiv:0810.4519] [INSPIRE].

[53] A. Amariti, D. Forcella and A. Mariotti, Integrability on the master space, JHEP 06 (2012) 053 [arXiv: 1203.1616] [INSPIRE].

[54] D. Martelli, J. Sparks and S.-T. Yau, Sasaki-Einstein manifolds and volume minimisation, Commun. Math. Phys. 280 (2008) 611 [hep-th/0603021] [INSPIRE].

[55] A. Butti, D. Forcella and A. Zaffaroni, Counting BPS baryonic operators in CFTs with Sasaki-Einstein duals, JHEP 06 (2007) 069 [hep-th/0611229] [INSPIRE].

[56] A. Goncharov and R. Kenyon, Dimers and cluster integrable systems, arXiv:1107.5588 [INSPIRE].

[57] R. Eager, S. Franco and K. Schaeffer, Dimer models and integrable systems, JHEP 06 (2012) 106 [arXiv:1107.1244] [INSPIRE].

[58] J. Golden, A.B. Goncharov, M. Spradlin, C. Vergu and A. Volovich, Motivic amplitudes and cluster coordinates, arXiv: 1305.1617 [INSPIRE].

[59] A. Hanany and R.-K. Seong, Brane tilings and specular duality, JHEP 08 (2012) 107 [arXiv:1206.2386] [INSPIRE].

[60] A. Hanany and R.-K. Seong, Brane tilings and reflexive polygons, Fortsch. Phys. 60 (2012) 695 [arXiv:1201.2614] [INSPIRE]. 